$$
\text { for }
$$

\title{
Nickel-Catalyzed Decarbonylative Cyanation of Acyl Chlorides
}

\author{
Zhenhua Wang, ${ }^{\dagger}$ Xiu Wang, ${ }^{\dagger}$ Yasuyuki Ura,${ }^{\dagger}$ and Yasushi Nishihara*, \\ ${ }^{\dagger}$ Graduate School of Natural Science and Technology, Okayama University, 3-1-1 \\ Tsushimanaka, Kita-ku, Okayama 700-8530, Japan \\ tDepartment of Chemistry, Biology, and Environmental Science, Faculty of Science, \\ Nara Women's University, Kitauoyani-shi-machi, Nara 630-8506, Japan \\ ${ }^{\S}$ Research Institute for Interdisciplinary Science, Okayama University, 3-1-1 \\ Tsushimanaka, Kita-ku, Okayama 700-8530, Japan \\ Phone: $+81-86-251-7855$ \\ Fax: $+81-86-251-7855$ \\ Email: ynishiha@okayama-u.ac.jp
}

1. General Considerations

2. Optimization Details

3. Ni-Catalyzed Decarbonylative Cyanation of Acyl Chlorides

S11-S16

4. Ni-Catalyzed Decarbonylative Cyanation of Carboxylic Acids

S17-S20

5. Ni-Catalyzed Decarbonylative Cyanation of Estrone Derivatives

S21-S23

6. Mechanistic Studies

$S 24-S 36$

7. Crystallographic Data of Complexes $\mathbf{1 0}$ and $\mathbf{1 3}$

S37-S57

8. Copies of NMR Spectra

S58-S84

9. References 


\section{General Considerations}

Unless otherwise noted, all the reactions were carried out under an argon atmosphere using standard Schlenk techniques. Glassware was dried in an oven $\left(150^{\circ} \mathrm{C}\right)$ and heated under reduced pressure prior to use. Solvents were employed as eluents for all other routine operation, as well as dehydrated solvent were purchased from commercial suppliers and employed without any further purification. For thin layer chromatography (TLC) analyses throughout this work, Merck precoated TLC plates (silica gel 60 GF254, $0.25 \mathrm{~mm}$ ) were used. Silica gel column chromatography was carried out using silica gel $60 \mathrm{~N}$ (spherical, neutral, 40-100 $\mu \mathrm{m}$ ) from Kanto Chemicals Co., Inc. NMR spectra $\left({ }^{1} \mathrm{H},{ }^{13} \mathrm{C}\left\{{ }^{1} \mathrm{H}\right\},{ }^{31} \mathrm{P}\left\{{ }^{1} \mathrm{H}\right\}\right.$ NMR, and $\left.{ }^{19} \mathrm{~F}\left\{{ }^{1} \mathrm{H}\right\}\right)$ were recorded on Varian INOVA-600 (600 MHz), Mercury-400 (400 MHz), or 300NMR ASW (300 MHz) spectrometers. GC analyses were performed on a Shimadzu GC-14A equipped with a flame ionization detector using Shimadzu Capillary Column (CBP1-M25-025) and Shimadzu CR6A-Chromatopac integrator. Infrared spectra were recorded on a Shimadzu IR Prestige-21 spectrophotometer. GC/MS analyses were carried out on a SHIMADZU GC-17A equipped with a SHIMADZU QP-5050 GCMS system. Elemental analyses were carried out with a Perkin-Elmer 2400 CHN elemental analyser at Okayama University.

All ${ }^{1} \mathrm{H}$ NMR chemical shifts were reported in ppm relative to proton resonance in $\mathrm{CDCl}_{3}$ at $\delta 7.26$, (CD) $)_{3} \mathrm{SO}$ at $\delta 2.50, \mathrm{CD}_{2} \mathrm{Cl}_{2}$ at $\delta 5.32,(\mathrm{CD})_{3} \mathrm{CO}$ at $\delta 2.05$. All ${ }^{13} \mathrm{C}\left\{{ }^{1} \mathrm{H}\right\}$ NMR chemical shifts were reported in ppm relative to carbon resonance in $\mathrm{CDCl}_{3}$ at $\delta 77.16,(\mathrm{CD})_{3} \mathrm{SO}$ at $\delta 39.52, \mathrm{CD}_{2} \mathrm{Cl}_{2}$ at $\delta$ 53.84, $(\mathrm{CD})_{3} \mathrm{CO}$ at $\delta 29.84$. The ${ }^{31} \mathrm{P}\left\{{ }^{1} \mathrm{H}\right\}$ chemical shifts were reported in ppm relative to external reference of $\mathrm{H}_{3} \mathrm{PO}_{4}$ at $\delta 0.00$. The ${ }^{19} \mathrm{~F}\left\{{ }^{1} \mathrm{H}\right\}$ NMR spectra were measured by using $\mathrm{CCl}_{3} \mathrm{~F}(\delta=0.00$ ppm) as an external standard. The NMR yields were determined using dibromomethane as an internal standard. The GC yields were determined by GC analysis of the crude mixture, using $n$-dodecane as an internal standard.

\section{Chemicals}

Unless otherwise noted, materials obtained from commercial suppliers were used without further purification. Benzoyl chloride 1a and trimethylsilyl cyanide 2 was purchased from TCI Co., Ltd. All acid chlorides were distilled before used. Bis(1,5-cyclooctadiene)nickel and triethylphosphine (1.0 M in THF) were purchased from Sigma-Aldrich Co. Triphenylphosphine was obtained from Nacalai Tesque,. $\quad n$-Dodecane was purchased from Kanto Chemical Co., Inc. 


\section{Optimization Details}

Table S1. Screening of Ligands

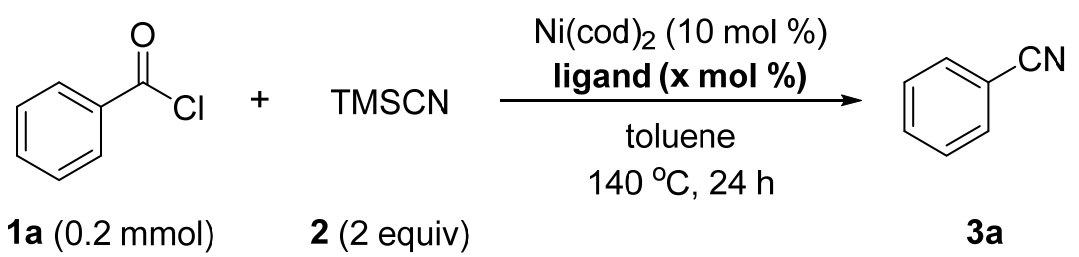

\begin{tabular}{|c|c|c|}
\hline Entry $^{a}$ & Ligand (x mol \%) & Yield of $\mathbf{3 a}(\%)^{b}$ \\
\hline 1 & DCyPE (20) & 5 \\
\hline 2 & DPPM (20) & 10 \\
\hline 3 & DPPE (20) & 80 \\
\hline 4 & DPPP (20) & 20 \\
\hline 5 & DPPF (20) & 49 \\
\hline 6 & Xantphos (20) & 44 \\
\hline 7 & $\mathrm{P}^{n} \mathrm{Bu}_{3}(40)$ & 0 \\
\hline 8 & $\mathrm{PCy}_{3}(40)$ & 2 \\
\hline 9 & $\mathrm{PPh}_{3}(40)$ & 85 \\
\hline $10^{c}$ & $\mathrm{PPh}_{3}(40)$ & 53 \\
\hline $11^{d}$ & $\mathrm{PPh}_{3}(40)$ & 56 \\
\hline $12^{e}$ & $\mathrm{PPh}_{3}(40)$ & 86 \\
\hline 13 & $\mathrm{PPh}_{3}(50)$ & 90 \\
\hline $14^{f}$ & $\mathrm{PPh}_{3}(\mathbf{4 0 )}$ & $>99$ \\
\hline
\end{tabular}

${ }^{a}$ Standard condition: 1a $(0.2 \mathrm{mmol}), 2(0.4 \mathrm{mmol})$ and $\mathrm{Ni}(\operatorname{cod})_{2}(0.02 \mathrm{mmol})$ in toluene $(1.0 \mathrm{~mL})$ at $140{ }^{\circ} \mathrm{C}$ for $24 \mathrm{~h} . \quad{ }^{b}$ Determined by GC analysis of the crude mixture, using $n$-dodecane as an internal standard. $\quad{ }^{c} \mathrm{KF}$ (2.5 equiv). $\quad{ }^{d}$ Toluene $(0.5 \mathrm{~mL}) . \quad{ }^{e} 150{ }^{\circ} \mathrm{C} . \quad{ }^{f} \mathrm{TMSCN}$ (1.2 equiv).

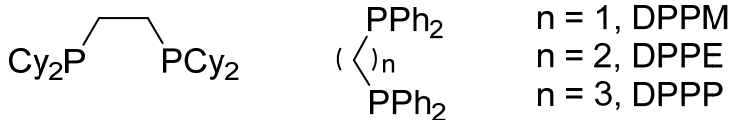

DCyPE

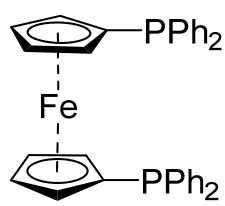

DPPF<smiles>CC1(C)c2cccc(P)c2Oc2c(P)cccc21</smiles>

Xantphos 
Table S2. Screening the Ratio of $\mathrm{Ni}(\operatorname{cod})_{2}$ and $\mathrm{PPh}_{3}$

\begin{tabular}{|c|c|c|c|}
\hline & $\begin{array}{l}+ \text { TMSCN } \\
\mathbf{2} \text { (1.2 equiv) }\end{array}$ & $\begin{array}{c}\mathbf{N i ( c o d})_{2} \\
\mathbf{P P h}_{3} \\
\text { toluene } \\
140^{\circ} \mathrm{C}, 24 \mathrm{~h}\end{array}$ & $3 a$ \\
\hline Entry $^{a}$ & $\mathrm{Ni}(\operatorname{cod})_{2}(\mathrm{~mol} \%)$ & $\mathrm{PPh}_{3}(\mathrm{~mol} \%)$ & Yield of $\mathbf{3 a}(\%)^{b}$ \\
\hline 1 & 0 & 40 & 0 \\
\hline 2 & 10 & 40 & $>99$ \\
\hline 3 & 10 & 30 & 95 \\
\hline 4 & 10 & 20 & 97 \\
\hline 5 & 10 & 10 & 91 \\
\hline 6 & 5 & 10 & 24 \\
\hline 7 & 2 & 4 & 0 \\
\hline 8 & 10 & 0 & 41 \\
\hline 9 & 0 & 0 & 0 \\
\hline
\end{tabular}

${ }^{a}$ Standard condition: $1 \mathrm{a}(0.2 \mathrm{mmol})$ and $2(0.24 \mathrm{mmol})$ in toluene $(1.0 \mathrm{~mL})$ at $140{ }^{\circ} \mathrm{C}$ for $24 \mathrm{~h}$.

${ }^{b}$ Determined by GC analysis of the crude mixture, using $n$-dodecane as an internal standard. 
Table S3. Screening of Temperature and Reaction Time

\begin{tabular}{|c|c|c|c|c|}
\hline & $1 \mathrm{a}(0.2 \mathrm{mmol})$ & $\begin{array}{l}\text { TMSCN } \\
\mathbf{2} \text { (1.2 equiv) }\end{array}$ & $\begin{array}{c}\mathrm{Ni}(\mathrm{cod})_{2}(10 \mathrm{~mol} \%) \\
\mathrm{PPh}_{3}(20 \mathrm{~mol} \%) \\
\text { toluene } \\
\text { temp. } \\
\text { time }\end{array}$ & $3 a$ \\
\hline Entry $^{a}$ & \multicolumn{2}{|c|}{ Temp. $\left({ }^{\circ} \mathrm{C}\right)$} & Time (h) & Yield of $\mathbf{3 a}(\%)^{b}$ \\
\hline 1 & \multicolumn{2}{|c|}{140} & 24 & 97 \\
\hline 2 & \multicolumn{2}{|c|}{130} & 24 & $>99$ \\
\hline 3 & \multicolumn{2}{|c|}{120} & 24 & 88 \\
\hline 4 & \multicolumn{2}{|c|}{110} & 24 & 88 \\
\hline 5 & \multicolumn{2}{|c|}{130} & 8 & $>99$ \\
\hline 6 & \multicolumn{2}{|c|}{130} & 3 & $>99$ \\
\hline 7 & \multicolumn{2}{|c|}{130} & 1 & $>99$ \\
\hline 8 & \multicolumn{2}{|c|}{120} & 1 & 89 \\
\hline 9 & \multicolumn{2}{|c|}{130} & $0.5(=30 \mathrm{~min})$ & $>99$ \\
\hline 10 & \multicolumn{2}{|c|}{130} & 0.17 (= $10 \mathrm{~min})$ & 99 \\
\hline
\end{tabular}

${ }^{a}$ Standard condition: 1a $(0.2 \mathrm{mmol}), 2(0.24 \mathrm{mmol}), \mathrm{Ni}(\mathrm{cod})_{2}(0.02 \mathrm{mmol})$ and $\mathrm{PPh}_{3}(0.04 \mathrm{mmol})$ in toluene $(1.0 \mathrm{~mL}) . \quad{ }^{b}$ Determined by GC analysis of the crude mixture, using $n$-dodecane as an internal standard. 
Table S4. Screening the 'CN' Sources<smiles>O=C(Cl)c1ccccc1</smiles>

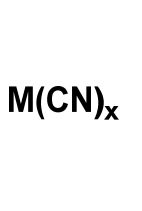

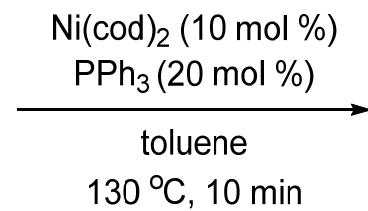<smiles>N#Cc1ccccc1</smiles>

1a $(0.2 \mathrm{mmol})$

(1.2 equiv)

3a

\begin{tabular}{clc}
\hline Entry $^{a}$ & 'CN' Source & Yield of 3a $(\%)^{b}$ \\
\hline 1 & $\mathrm{~K}_{4} \mathrm{Fe}(\mathrm{CN})_{6}$ & 0 \\
2 & $\mathrm{NaCN}$ & 0 \\
3 & $\mathrm{KCN}$ & 0 \\
4 & $\mathrm{CuCN}$ & 5 \\
5 & $\mathrm{Zn}(\mathrm{CN})_{2}$ & 3 \\
6 & $\mathrm{EtOC}=\mathrm{OCN}$ & 0 \\
\hline
\end{tabular}

a Standard condition: 1a $(0.2 \mathrm{mmol})$, 'CN' Sources $(0.24 \mathrm{mmol}), \mathrm{Ni}(\mathrm{cod})_{2}(0.02 \mathrm{mmol})$ and $\mathrm{PPh}_{3}$ $(0.04 \mathrm{mmol})$ in toluene $(1.0 \mathrm{~mL})$ at $130{ }^{\circ} \mathrm{C}$ for $10 \mathrm{~min} .{ }^{b}$ Determined by GC analysis of the crude mixture, using $n$-dodecane as an internal standard.

Table S5. Screening the Volume of Toluene
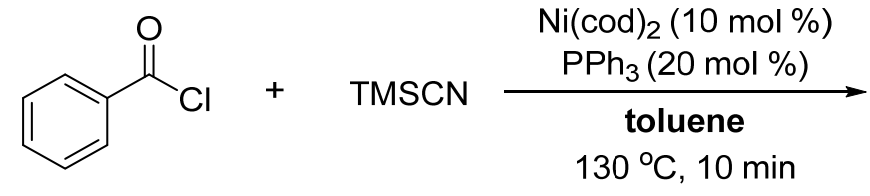

$1 \mathrm{a}(0.2 \mathrm{mmol})$

2 (1.2 equiv)<smiles>N#Cc1ccccc1</smiles>

$3 a$

\begin{tabular}{ccc}
\hline Entry $^{a}$ & Amount of toluene $(\mathrm{mL})$ & Yield of 3a (\%) $^{b}$ \\
\hline $\mathbf{1}$ & $\mathbf{1 . 0}$ & $\mathbf{9 9}$ \\
2 & 0.5 & 66 \\
3 & 0 & 0 \\
\hline
\end{tabular}

${ }^{a}$ Standard condition: 1a $(0.2 \mathrm{mmol}), 2(0.24 \mathrm{mmol}), \mathrm{Ni}(\mathrm{cod})_{2}(0.02 \mathrm{mmol}), \mathrm{PPh}_{3}(0.04 \mathrm{mmol})$ in toluene at $130{ }^{\circ} \mathrm{C}$ for $10 \mathrm{~min} . \quad{ }^{b}$ Determined by GC analysis of the crude mixture, using $n$-dodecane as an internal standard. 
Table S6. Time Course Experiments of Ni-Catalyzed Decarbonylative Cyanation of Benzoyl Chloride (1a) with 2 at $110{ }^{\circ} \mathrm{C}$

\begin{tabular}{|c|c|c|c|}
\hline a $(0.2 \mathrm{mmol})$ & $\begin{array}{l}\mathrm{NMSCN} \\
\mathbf{2} \text { (1.2 equiv) }\end{array}$ & $\begin{array}{l}\mathrm{d})_{2}(10 \mathrm{~mol} \%) \\
\mathrm{h}_{3}(20 \mathrm{~mol} \%) \\
\text { toluene } \\
110{ }^{\circ} \mathrm{C} \\
\text { time }\end{array}$ & $3 a$ \\
\hline Entry $^{a}$ & Time (h) & & Yield of $\mathbf{3 a}(\%)^{b}$ \\
\hline 1 & $0.17(=10 \mathrm{~min})$ & & 55 \\
\hline 2 & $0.5(=30 \mathrm{~min})$ & & 83 \\
\hline 3 & 1 & & 87 \\
\hline 4 & 3 & & 87 \\
\hline 5 & 6 & & 88 \\
\hline 6 & 12 & & 88 \\
\hline 7 & 24 & & 88 \\
\hline
\end{tabular}

${ }^{a}$ Standard condition: 1a $(0.2 \mathrm{mmol}), 2(0.24 \mathrm{mmol}), \mathrm{Ni}(\operatorname{cod})_{2}(0.02 \mathrm{mmol})$ and $\mathrm{PPh}_{3}(0.04 \mathrm{mmol})$ in toluene $(1 \mathrm{~mL})$ at $110^{\circ} \mathrm{C} . \quad{ }^{b}$ Determined by GC analysis of the crude mixture, using $n$-dodecane as an internal standard. 
Table S7. The Effect of Substituents and Temperature on the Yield of 3

\begin{tabular}{|c|c|c|c|c|}
\hline & $\mathrm{m}_{\mathrm{Cl}}^{\mathrm{O}}$ & $\begin{array}{c}\text { TMSCN } \\
\mathbf{2} \text { (1.2 equiv) }\end{array}$ & $\begin{array}{c}\mathrm{Ni}(\mathrm{cod})_{2}(10 \mathrm{~mol} \%) \\
\mathrm{PPh}_{3}(20 \mathrm{~mol} \%)\end{array}$ & 3 \\
\hline Entry $^{a}$ & $\mathrm{R}$ & Temp. & Time (h) & Yield of $3(\%)$ \\
\hline 1 & $\mathrm{CF}_{3}$ & 110 & 1 & $96(94)$ \\
\hline 2 & $\mathrm{CF}_{3}$ & 130 & $0.17(=10 \mathrm{~min})$ & 99 \\
\hline 3 & $\mathrm{OMe}$ & 110 & 1 & 70 \\
\hline 4 & $\mathrm{OMe}$ & 130 & 1 & 74 \\
\hline 5 & OMe & 150 & 1 & $88(84)$ \\
\hline 6 & I & 80 & 1 & $98(92)$ \\
\hline 7 & I & 100 & 1 & 75 \\
\hline 8 & I & 110 & 1 & 60 \\
\hline
\end{tabular}

${ }^{a}$ Standard condition: 1 (0.2 mmol), 2 (0.24 mmol), Ni(cod) $2(0.02 \mathrm{mmol}), \mathrm{PPh}_{3}(0.04 \mathrm{mmol})$ in toluene $(1 \mathrm{~mL})$. ${ }^{b}$ Determined by GC analysis of the crude mixture, using $n$-dodecane as an internal standard, isolated yields in parentheses. 
Table S8. Effect of Various Transition-Metal Catalysts

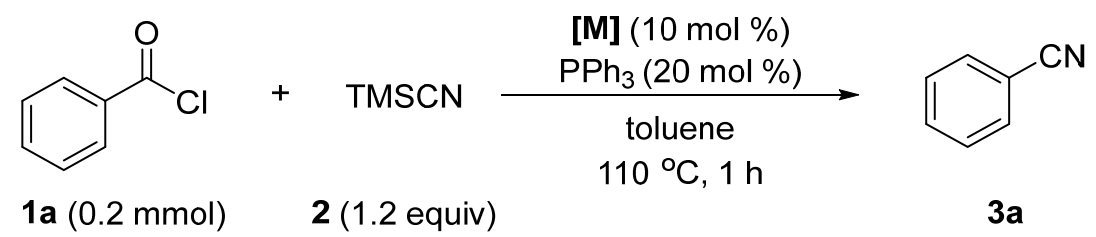

\begin{tabular}{|c|c|c|}
\hline Entry $^{a}$ & {$[\mathrm{M}]$} & Yield of $\mathbf{3 a}(\%)^{b}$ \\
\hline 1 & $\mathrm{Ni}(\operatorname{cod})_{2}$ & 87 \\
\hline 2 & $\mathrm{NiCl}_{2}$ & 0 \\
\hline 3 & $\mathrm{NiBr}_{2}$ & 0 \\
\hline 4 & $\mathrm{Ni}(\mathrm{OAc})_{2} \cdot 4 \mathrm{H}_{2} \mathrm{O}$ & 0 \\
\hline 5 & $\mathrm{Ni}(\mathrm{acac})_{2}$ & 0 \\
\hline 6 & $\mathrm{Pd}(\mathrm{OAc})_{2}$ & 0 \\
\hline $7^{c}$ & $\mathrm{Pd}\left(\mathrm{PPh}_{3}\right)_{4}$ & 4 \\
\hline
\end{tabular}

${ }^{a}$ Standard condition: 1a $(0.2 \mathrm{mmol}), 2(0.24 \mathrm{mmol})$ and $\mathrm{PPh}_{3}(0.04 \mathrm{mmol})$ in toluene $(1 \mathrm{~mL})$ at $110^{\circ} \mathrm{C}$ for $1 \mathrm{~h}$. $\quad{ }^{b}$ Determined by GC analysis of the crude mixture, using $n$-dodecane as an internal standard. ${ }^{c}$ Without $\mathrm{PPh}_{3}$.

Table S9. Screening of Solvents

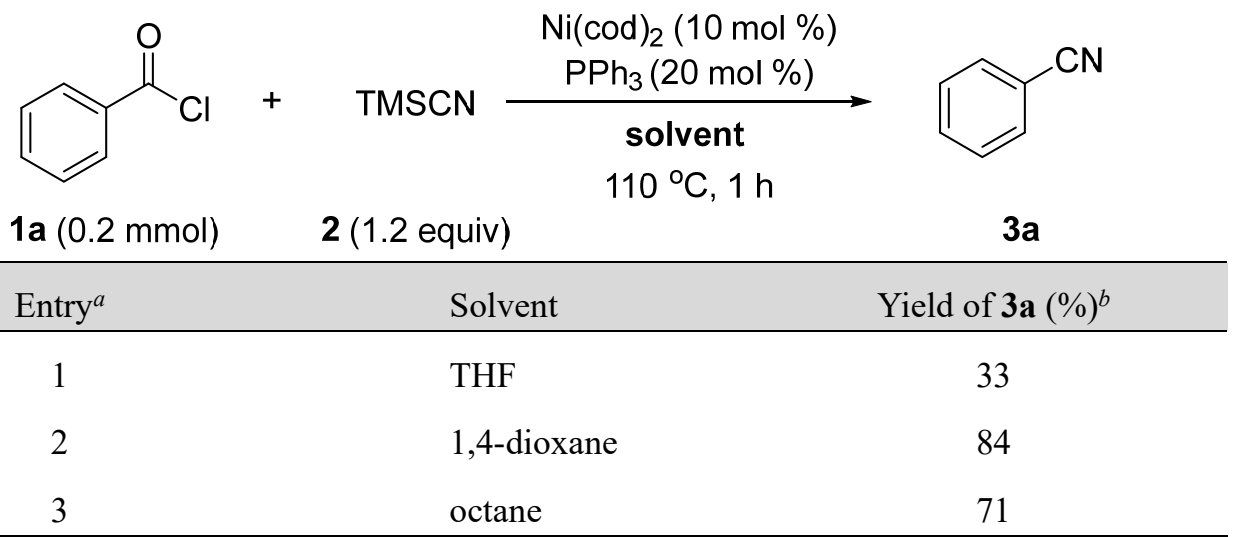

${ }^{a}$ Standard condition: 1a $(0.2 \mathrm{mmol}), 2(0.24 \mathrm{mmol}), \mathrm{Ni}(\mathrm{cod})_{2}(0.02 \mathrm{mmol})$ and $\mathrm{PPh}_{3}(0.04 \mathrm{mmol})$ at $110{ }^{\circ} \mathrm{C}$ for $1 \mathrm{~h} . \quad{ }^{b}$ Determined by GC analysis of the crude mixture, using $n$-dodecane as an internal standard. 
Table S10. Deviation from Standard Conditions

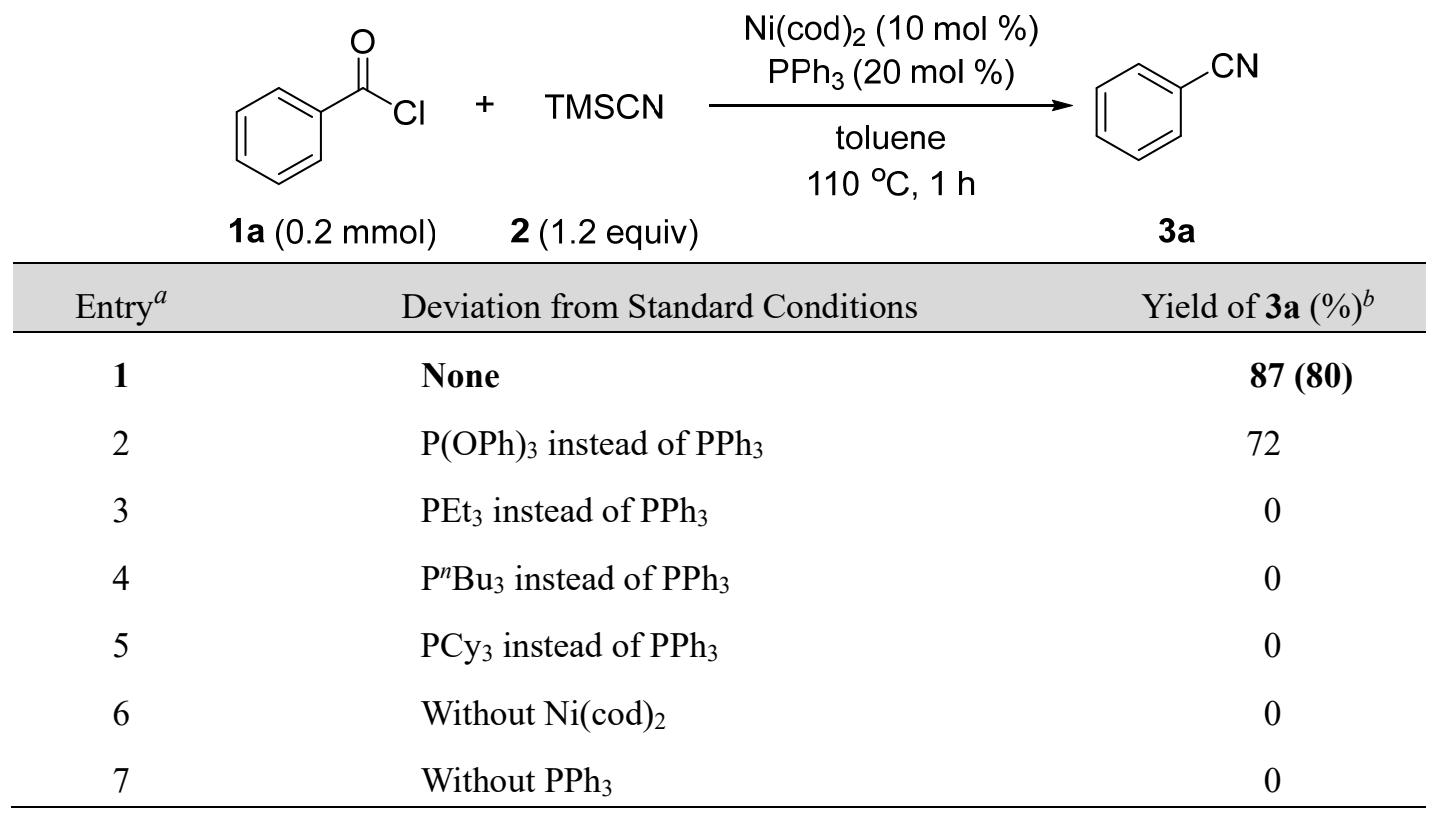

${ }^{a}$ Standard condition: 1a $(0.2 \mathrm{mmol}), \mathbf{2 a}(0.24 \mathrm{mmol}), \mathrm{Ni}(\operatorname{cod})_{2}(0.02 \mathrm{mmol}), \mathrm{PPh}_{3}(0.04 \mathrm{mmol})$, toluene $(1 \mathrm{~mL}), 110{ }^{\circ} \mathrm{C}, 1 \mathrm{~h} . \quad{ }^{b}$ Determined by GC analysis using $n$-dodecane as an internal standard. An isolated yield is given in parentheses. 


\section{Ni-Catalyzed Decarbonylative Cyanation of Commercially Available Acyl Chlorides}

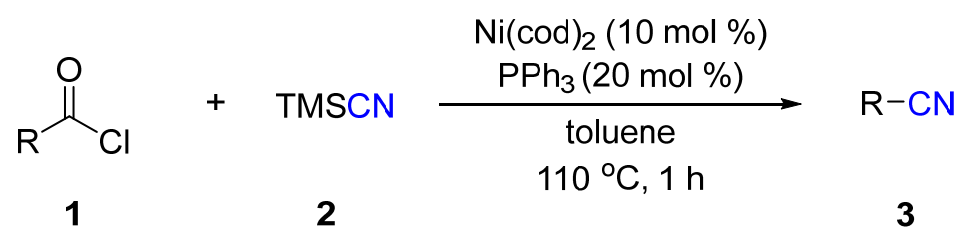

To a toluene $(1 \mathrm{~mL})$ solution of $\mathrm{Ni}(\mathrm{cod})_{2}(5.5 \mathrm{mg}, 0.02 \mathrm{mmol}, 10 \mathrm{~mol} \%)$ and $\mathrm{PPh}_{3}(10.5 \mathrm{mg}, 0.04$ mmol, $20 \mathrm{~mol} \%$ ) in an oven-dried Schlenk tube containing a stirring bar, were added acyl chlorides 1 $(0.2 \mathrm{mmol})$ and TMSCN (2) $\left(23.4 \mathrm{mg}, 0.24 \mathrm{mmol}, 1.2\right.$ equiv). The mixture was heated at $110^{\circ} \mathrm{C}$ with stirring for $1 \mathrm{~h}$. After cooling to room temperature, the crude product was purified by column chromatography on silica gel or bulb-to-bulb distillation to afford the corresponding nitriles 3 .

A representative procedure at 1-mmol scale: to a toluene $(5 \mathrm{~mL})$ solution of $\mathrm{Ni}(\mathrm{cod})_{2}(27.5 \mathrm{mg}, 0.1$ mmol, $10 \mathrm{~mol} \%$ ) and $\mathrm{PPh}_{3}(52.5 \mathrm{mg}, 0.20 \mathrm{mmol}, 20 \mathrm{~mol} \%$ ) in an oven-dried Schlenk tube containing a stirring bar, were added benzoyl chloride 1a (140.6 mg, $1.0 \mathrm{mmol})$ and TMSCN (2) (119.1 mg, 1.2 mmol, 1.2 equiv). The mixture was heated at $110^{\circ} \mathrm{C}$ with stirring for $1 \mathrm{~h}$. After cooling to room temperature, the crude product was purified by bulb-to-bulb distillation to afford the corresponding nitrile 3a in $65 \%$ yield $(67.0 \mathrm{mg})$.

\section{Benzonitrile (3a) ${ }^{1}$}<smiles>N#Cc1ccccc1</smiles>

Purification: bulb-to-bulb distillation $\left(60^{\circ} \mathrm{C} / 10 \mathrm{mmHg}\right)$. Colorless oil. Yield was $80 \%(16.6 \mathrm{mg})$. ${ }^{1} \mathrm{H}$ NMR $\left(600 \mathrm{MHz}, \mathrm{CDCl}_{3}\right): \delta$ 7.45-7.47 (m, 2H), 7.58-7.61 (m, 1H), $7.64(\mathrm{~d}, J=8.2 \mathrm{~Hz}, 2 \mathrm{H})$.

\section{4-Methylbenzonitrile (3b) ${ }^{1}$}<smiles>Cc1ccc(C#N)cc1</smiles>

Purification: bulb-to-bulb distillation $\left(80^{\circ} \mathrm{C} / 10 \mathrm{mmHg}\right)$. Colorless oil. Yield was $90 \%(21.1 \mathrm{mg})$. ${ }^{1} \mathrm{H}$ NMR $\left(600 \mathrm{MHz}, \mathrm{CDCl}_{3}\right): \delta 2.40(\mathrm{~s}, 3 \mathrm{H}), 7.25(\mathrm{~d}, J=8.2 \mathrm{~Hz}, 2 \mathrm{H}), 7.53(\mathrm{~d}, J=8.2 \mathrm{~Hz}, 2 \mathrm{H})$.

\section{4-Butylbenzonitrile (3c) ${ }^{2}$}


CN

Purification: column chromatography (hexane/EtOAc $=40: 1, R_{\mathrm{f}}=0.37$ ). Colorless oil. Yield was 94\% (29.9 mg). $\quad{ }^{1} \mathrm{H}$ NMR (600 MHz, $\left.\mathrm{CDCl}_{3}\right): \delta 0.93$ (t, $\left.J=7.4 \mathrm{~Hz}, 3 \mathrm{H}\right), 1.32-1.37$ (m, 2H), 1.58-1.61 (m, 2H), $2.66(\mathrm{t}, J=7.2 \mathrm{~Hz}, 2 \mathrm{H}), 7.27(\mathrm{~d}, J=8.2 \mathrm{~Hz}, 2 \mathrm{H}), 7.53-7.58(\mathrm{~d}, J=8.2 \mathrm{~Hz}, 2 \mathrm{H})$.

\section{4-(Tert-butyl)benzonitrile (3d) ${ }^{3}$}<smiles>N#Cc1ccc(Br)cc1</smiles>

Purification: bulb-to-bulb distillation $\left(105^{\circ} \mathrm{C} / 10 \mathrm{mmHg}\right)$. Colorless oil. Yield was $96 \%$ (30.6 mg). ${ }^{1} \mathrm{H}$ NMR (600 MHz, $\left.\mathrm{CDCl}_{3}\right): \delta 1.33$ (s, 9H), $7.48(\mathrm{~d}, J=8.4 \mathrm{~Hz}, 2 \mathrm{H}), 7.59(\mathrm{~d}, J=8.4 \mathrm{~Hz}, 2 \mathrm{H})$.

\section{$\left[1,1^{\prime}\right.$-Biphenyl]-4-carbonitrile $(3 \mathrm{e})^{1}$}<smiles>N#Cc1ccc(-c2ccccc2)cc1</smiles>

Purification: column chromatography (hexane/EtOAc $=40: 1, R_{\mathrm{f}}=0.32$ ). White solid. Yield was $80 \%$ (28.7 mg). ${ }^{1} \mathrm{H}$ NMR (600 MHz, $\left.\mathrm{CDCl}_{3}\right): \delta$ 7.41-7.44 (m, 1H), 7.47-7.50 (m, 2H), 7.58-7.60 (m, 2H), 7.68-7.70 (m, 2H), 7.72-7.74 (m, 2H).

\section{4-Methoxybenzonitrile (3f) ${ }^{1}$}<smiles>COc1ccc(C#N)cc1</smiles>

Purification: column chromatography (hexane/EtOAc $=20: 1, R_{\mathrm{f}}=0.39$ ). White solid. Yield was $84 \%$ (22.4 mg). $\quad{ }^{1} \mathrm{H}$ NMR (600 MHz, $\left.\mathrm{CDCl}_{3}\right): \delta 3.86(\mathrm{~s}, 3 \mathrm{H}), 6.95(\mathrm{~d}, J=9.0 \mathrm{~Hz}, 2 \mathrm{H}), 7.59$ (d, $J=9.0 \mathrm{~Hz}$, $2 \mathrm{H})$.

\section{4-Butoxybenzonitrile (3g) ${ }^{4}$}<smiles>CCCCOc1ccc(C#N)cc1</smiles>

Purification: column chromatography (hexane/EtOAc $=40: 1, R_{\mathrm{f}}=0.35$ ). Colorless oil. Yield was 
84\% (29.4 mg). $\quad{ }^{1} \mathrm{H}$ NMR (600 MHz, $\left.\mathrm{CDCl}_{3}\right): \delta 0.98(\mathrm{t}, J=7.4 \mathrm{~Hz}, 3 \mathrm{H}), 1.46-1.52(\mathrm{~m}, 2 \mathrm{H}), 1.76-1.81$ (m, 2H), $4.00(\mathrm{t}, J=6.5 \mathrm{~Hz}, 2 \mathrm{H}), 6.93(\mathrm{~d}, J=8.9 \mathrm{~Hz}, 2 \mathrm{H}), 7.56(\mathrm{~d}, J=8.9 \mathrm{~Hz}, 2 \mathrm{H})$.

\section{Methyl 4-cyanobenzoate (3k) ${ }^{1}$}<smiles>COC(=O)c1ccc(C#N)cc1</smiles>

Purification: column chromatography (hexane/EtOAc $=20: 1, R_{\mathrm{f}}=0.40$ ). White solid. Yield was $88 \%$ (28.4 mg). $\quad{ }^{1} \mathrm{H}$ NMR (400 MHz, $\left.\mathrm{CDCl}_{3}\right): \delta 3.95$ (s, 3H), 7.71-7.76 (d, $\left.J=8.4 \mathrm{~Hz}, 2 \mathrm{H}\right), 8.11-8.15$ (d, $J$ $=8.4 \mathrm{~Hz}, 2 \mathrm{H})$.

\section{4-(Trifluoromethyl)benzonitrile (3n) ${ }^{1}$}<smiles>N#Cc1ccc(C(F)(F)F)cc1</smiles>

Purification: bulb-to-bulb distillation $\left(60^{\circ} \mathrm{C} / 10 \mathrm{mmHg}\right)$. Colorless oil. Yield was $94 \%$ (32.2 $\left.\mathrm{mg}\right)$. ${ }^{1} \mathrm{H}$ NMR $\left(600 \mathrm{MHz}, \mathrm{CDCl}_{3}\right): \delta 7.76(\mathrm{~d}, J=7.8 \mathrm{~Hz}, 2 \mathrm{H}), 7.79-7.83(\mathrm{~d}, J=7.8 \mathrm{~Hz}, 2 \mathrm{H})$.

\section{Terephthalonitrile (3o) ${ }^{1}$}<smiles>N#Cc1ccc(C#N)cc1</smiles>

Purification: column chromatography (hexane/ $\mathrm{EtOAc}=10: 1, R_{\mathrm{f}}=0.32$ ). White solid. Yield was $82 \%$ (21.0 mg). $\quad{ }^{1} \mathrm{H}$ NMR (400 MHz, $\left.\mathrm{CDCl}_{3}\right): \delta 7.80(\mathrm{~s}, 4 \mathrm{H})$.

\section{4-Fluorobenzonitrile (3p) ${ }^{1}$}<smiles>N#Cc1ccc(F)cc1</smiles>

Purification: bulb-to-bulb distillation $\left(55^{\circ} \mathrm{C} / 10 \mathrm{mmHg}\right)$. Colorless oil. Yield was $85 \%$ (20.6 mg) ${ }^{1} \mathrm{H}$ NMR (600 MHz, $\left.\mathrm{CDCl}_{3}\right): \delta 7.16-7.20(\mathrm{~m}, 2 \mathrm{H}), 7.65-7.70(\mathrm{~m}, 2 \mathrm{H})$.

\section{4-Chlorobenzonitrile (3q) $)^{5}$}


$\overbrace{}^{C N}$

Purification: bulb-to-bulb distillation $\left(90{ }^{\circ} \mathrm{C} / 10 \mathrm{mmHg}\right.$ ). White solid. Yield was $96 \%$ (26.4 $\left.\mathrm{mg}\right)$. ${ }^{1} \mathrm{H}$ NMR (600 MHz, $\left.\mathrm{CDCl}_{3}\right): \delta$ 7.45-7.48 (m, 2H), 7.58-7.61 (m, 2H).

\section{4-Bromobenzonitrile $(3 \mathbf{r})^{5}$}<smiles>N#Cc1ccc(Br)cc1</smiles>

Purification: bulb-to-bulb distillation $\left(90^{\circ} \mathrm{C} / 5 \mathrm{mmHg}\right)$. White solid. Yield was $93 \%(33.9 \mathrm{mg}) . \quad{ }^{1} \mathrm{H}$ $\operatorname{NMR}\left(600 \mathrm{MHz}, \mathrm{CDCl}_{3}\right): \delta 7.50-7.55(\mathrm{~m}, 2 \mathrm{H}), 7.62-7.65(\mathrm{~m}, 2 \mathrm{H})$.

\section{4-Iodobenzonitrile (3s) $)^{5}$}<smiles>N#Cc1ccc(I)cc1</smiles>

Purification: bulb-to-bulb distillation $\left(115^{\circ} \mathrm{C} / 5 \mathrm{mmHg}\right)$. White solid. Yield was $92 \%(42.1 \mathrm{mg})$. ${ }^{1} \mathrm{H}$ NMR (600 MHz, $\left.\mathrm{CDCl}_{3}\right): \delta$ 7.35-7.39 (m, 2H), 7.83-7.87 (m, 2H).

\section{3-Methylbenzonitrile (3t) ${ }^{1}$}<smiles>Cc1cccc(C#N)c1</smiles>

Purification: bulb-to-bulb distillation $\left(75^{\circ} \mathrm{C} / 5 \mathrm{mmHg}\right)$. Colorless oil. Yield was $86 \%(20.1 \mathrm{mg})$. ${ }^{1} \mathrm{H}$ NMR (600 MHz, $\left.\mathrm{CDCl}_{3}\right): \delta 2.39$ (s, 3H), 7.35 (t, $\left.J=8.4 \mathrm{~Hz}, 1 \mathrm{H}\right), 7.39-7.41(\mathrm{~d}, J=8.4 \mathrm{~Hz}, 1 \mathrm{H})$, 7.44-7.47 (m, 2H).

\section{2-Methylbenzonitrile (3u) ${ }^{1}$}<smiles>Cc1ccccc1C#N</smiles>

Purification: bulb-to-bulb distillation $\left(70{ }^{\circ} \mathrm{C} / 5 \mathrm{mmHg}\right.$ ). Colorless oil. Yield was $88 \%$ (20.6 mg). ${ }^{1} \mathrm{H}$ NMR $\left(600 \mathrm{MHz}, \mathrm{CDCl}_{3}\right): \delta 2.55(\mathrm{~s}, 3 \mathrm{H}), 7.25-7.29(\mathrm{t}, J=7.8,0.6 \mathrm{~Hz}, 1 \mathrm{H}), 7.32(\mathrm{td}, J=7.8,0.6 \mathrm{~Hz}$, 
$1 \mathrm{H}), 7.48(\mathrm{td}, J=7.8,1.2 \mathrm{~Hz}, 1 \mathrm{H}), 7.60(\mathrm{dd}, J=7.8,1.2 \mathrm{~Hz}, 1 \mathrm{H})$.

\section{2-(Trifluoromethyl)benzonitrile (3v) ${ }^{6}$}<smiles>N#Cc1ccccc1C(F)(F)F</smiles>

Purification: bulb-to-bulb distillation $\left(70{ }^{\circ} \mathrm{C} / 5 \mathrm{mmHg}\right)$. Yellow liquid. Yield was $85 \%$ (29.1 $\left.\mathrm{mg}\right)$. ${ }^{1} \mathrm{H}$ NMR $\left(600 \mathrm{MHz}, \mathrm{CDCl}_{3}\right): \delta 7.70$ (td, $\left.J=7.6,0.6 \mathrm{~Hz}, 1 \mathrm{H}\right), 7.75$ (td, $\left.J=7.6,0.6 \mathrm{~Hz} 1 \mathrm{H}\right), 7.81$ (dd, $J$ $=7.8,0.6 \mathrm{~Hz}, 1 \mathrm{H}), 7.86(\mathrm{dd}, J=7.8,0.6 \mathrm{~Hz}, 1 \mathrm{H})$.

\section{2,4,6-Trimethylbenzonitrile $(3 w)^{3}$}<smiles>Cc1cc(C)c(C#N)c(C)c1</smiles>

Purification: column chromatography (hexane/EtOAc $=40: 1, R_{\mathrm{f}}=0.48$ ). White solid. Yield was $94 \%$ (27.3 mg). $\quad{ }^{1} \mathrm{H} \mathrm{NMR}\left(600 \mathrm{MHz}, \mathrm{CDCl}_{3}\right): \delta 2.32(\mathrm{~s}, 3 \mathrm{H}), 2.48(\mathrm{~s}, 6 \mathrm{H}), 6.93(\mathrm{~s}, 2 \mathrm{H})$.

\section{1-Naphthonitrile (3y) $)^{3}$}<smiles>N#Cc1cccc2ccccc12</smiles>

Purification: column chromatography (hexane/EtOAc $=40: 1, R_{\mathrm{f}}=0.35$ ). White solid. Yield was $82 \%$ (25.1 mg). $\quad{ }^{1} \mathrm{H} \mathrm{NMR}\left(600 \mathrm{MHz}, \mathrm{CDCl}_{3}\right): \delta 7.53(\mathrm{td}, J=7.5,1.2 \mathrm{~Hz}, 1 \mathrm{H}), 7.63(\mathrm{td}, J=7.8,1.2 \mathrm{~Hz}, 1 \mathrm{H})$, $7.71(\mathrm{td}, J=7.8,1.8 \mathrm{~Hz}, 1 \mathrm{H}), 7.93(\mathrm{td}, J=7.2,1.2 \mathrm{~Hz} 2 \mathrm{H}), 8.09$ (d, $J=8.3 \mathrm{~Hz}, 1 \mathrm{H}), 8.25$ (dd, $J=8.4$, $0.6 \mathrm{~Hz} 1 \mathrm{H})$.

\section{2-Naphthonitrile (3z) ${ }^{3}$}<smiles>N#Cc1ccc2ccccc2c1</smiles>

Purification: column chromatography (hexane/EtOAc $=40: 1, R_{\mathrm{f}}=0.34$ ). White solid. Yield was $80 \%$ (24.5 mg). ${ }^{1} \mathrm{H}$ NMR (600 MHz, $\left.\mathrm{CDCl}_{3}\right): \delta$ 7.60-7.67 (m, 3H), 7.89-7.93 (m, 3H), $8.24(\mathrm{~s}, 1 \mathrm{H})$. 


\section{Thiophene-2-carbonitrile (3aa) ${ }^{3}$}<smiles>N#Cc1cccs1</smiles>

Purification: bulb-to-bulb distillation $\left(50^{\circ} \mathrm{C} / 5 \mathrm{mmHg}\right)$. White solid. Yield was 99\% (21.6 mg). ${ }^{1} \mathrm{H}$ NMR (600 MHz, $\left.\mathrm{CDCl}_{3}\right): \delta$ 7.11-7.14 (m, 1H), 7.58-7.64 (m, 2H).

\section{Cyclohexanecarbonitrile (3ad) ${ }^{7}$}<smiles>N#CC1CCCCC1</smiles>

Purification: bulb-to-bulb distillation $\left(55^{\circ} \mathrm{C} / 5 \mathrm{mmHg}\right)$. Colorless oil. Yield was $86 \%$ (18.8 mg). ${ }^{1} \mathrm{H}$ NMR (600 MHz, $\left.\mathrm{CDCl}_{3}\right): \delta 1.41-1.53(\mathrm{~m}, 4 \mathrm{H}), 1.66-1.75(\mathrm{~m}, 4 \mathrm{H}), 1.83-1.85(\mathrm{~m}, 2 \mathrm{H}), 2.60-2.63(\mathrm{~m}$, $1 \mathrm{H})$.

\section{Adamantane-1-carbonitrile (3ae) ${ }^{8}$}

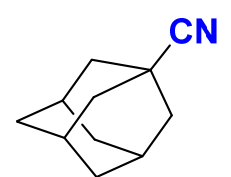

Purification: bulb-to-bulb distillation $\left(120^{\circ} \mathrm{C} / 5 \mathrm{mmHg}\right)$. White solid. Yield was $88 \%$ (28.4 $\left.\mathrm{mg}\right)$. ${ }^{1} \mathrm{H}$ NMR (600 MHz, $\left.\mathrm{CDCl}_{3}\right): \delta 1.69-1.74(\mathrm{~m}, 6 \mathrm{H}), 2.03(\mathrm{~s}, 9 \mathrm{H})$. 


\section{Ni-Catalyzed Decarbonylative Cyanation of Acyl Chlorides 1 in-situ Prepared from Carboxylic}

Acids

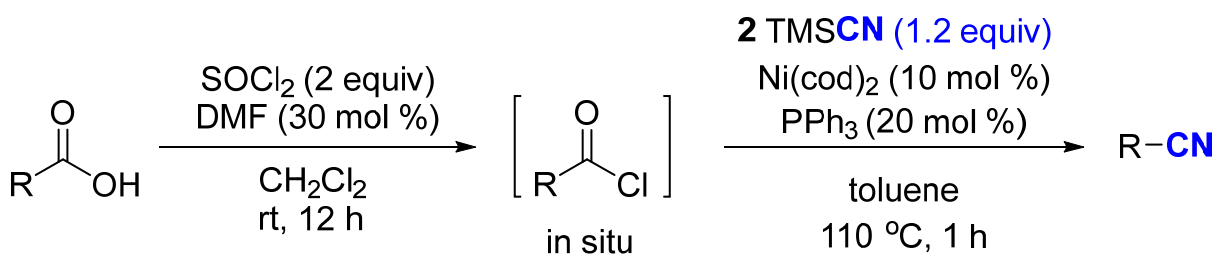

1

To the solution of anhydrous 1,2-dichloromethane $(0.8 \mathrm{~mL})$ and DMF $(5 \mu \mathrm{L})$ of carboxylic acid $(0.2$ mmol) in an oven-dried Schlenk tube containing a stirring bar, was added dropwise thionyl chloride (47.6 mg, $0.4 \mathrm{mmol}, 2$ equiv) using a syringe. The reaction mixture was stirred at room temperature for $12 \mathrm{~h}$. The solvent and unreacted thionyl chloride were removed under reduced pressure. The resulting acyl chloride was used without additional purification. ${ }^{9} \quad$ Then $\mathrm{Ni}(\operatorname{cod})_{2}(5.5 \mathrm{mg}, 0.02 \mathrm{mmol}$, $10 \mathrm{~mol} \%), \mathrm{PPh}_{3}(10.5 \mathrm{mg}, 0.04 \mathrm{mmol}, 20.0 \mathrm{~mol} \%)$, toluene (1 mL), and TMSCN (23.4 mg, $0.24 \mathrm{mmol}$, 1.2 equiv) were successively added. After the mixture was stirred at $110^{\circ} \mathrm{C}$ for $1 \mathrm{~h}$, the crude product was purified by column chromatography on silica gel to afford the corresponding nitriles 3 .

\section{4-(Benzyloxy)benzonitrile (3h) ${ }^{10}$}<smiles>N#Cc1ccc(OBr)cc1</smiles>

Purification: column chromatography (hexane/EtOAc $=20: 1, R_{\mathrm{f}}=0.39$ ). White solid. Yield was $90 \%$ (37.7 mg). $\quad{ }^{1} \mathrm{H}$ NMR (600 MHz, $\left.\mathrm{CDCl}_{3}\right): \delta 5.12(\mathrm{~s}, 2 \mathrm{H}), 7.01-7.03(\mathrm{~m}, 2 \mathrm{H}), 7.36-7.38(\mathrm{~m}, 1 \mathrm{H}), 7.39$ $7.43(\mathrm{~m}, 4 \mathrm{H}), 7.58-7.60(\mathrm{~m}, 2 \mathrm{H})$.

\section{Benzo[d][1,3]dioxole-5-carbonitrile (3i) ${ }^{2}$}<smiles>N#Cc1ccc2c(c1)OCO2</smiles>

Purification: column chromatography (hexane/EtOAc $\left.=20: 1, R_{\mathrm{f}}=0.40\right) . \quad$ White solid. $\quad$ Yield was 93\% (27.4 mg). $\left.\quad{ }^{1} \mathrm{H} \mathrm{NMR} \mathrm{(600} \mathrm{MHz,} \mathrm{CDCl}_{3}\right): \delta 6.07(\mathrm{~s}, 2 \mathrm{H}), 6.86(\mathrm{~d}, J=8.0 \mathrm{~Hz}, 1 \mathrm{H}), 7.03(\mathrm{~d}, J=1.6 \mathrm{~Hz}$, $1 \mathrm{H}), 7.21(\mathrm{dd}, J=8.0,1.6 \mathrm{~Hz}, 1 \mathrm{H})$.

\section{4-(Methylthio)benzonitrile (3j) ${ }^{11}$}


$\mathrm{MeS}^{\mathrm{CN}}$

Purification: column chromatography (hexane/EtOAc $=20: 1, R_{\mathrm{f}}=0.47$ ). Colorless oil. Yield was 98\% (29.3 mg). $\left.\quad{ }^{1} \mathrm{H} \mathrm{NMR} \mathrm{(600} \mathrm{MHz,} \mathrm{CDCl}_{3}\right): \delta 2.51(\mathrm{~s}, 3 \mathrm{H}), 7.26(\mathrm{~d}, J=8.6 \mathrm{~Hz}, 2 \mathrm{H}), 7.53(\mathrm{~d}, J=8.6$ $\mathrm{Hz}, 2 \mathrm{H})$.

\section{Phenyl 4-cyanobenzoate (3I) ${ }^{1}$}<smiles>N#Cc1ccc(C(=O)Oc2ccccc2)cc1</smiles>

Purification: column chromatography (hexane/EtOAc $=20: 1, R_{\mathrm{f}}=0.40$ ). White solid. Yield was $93 \%$ (41.5 mg). $\quad{ }^{1} \mathrm{H} \mathrm{NMR}\left(600 \mathrm{MHz}, \mathrm{CDCl}_{3}\right): \delta 7.21-7.23(\mathrm{~m}, 2 \mathrm{H}), 7.30-7.33(\mathrm{~m}, 1 \mathrm{H}), 7.44-7.47(\mathrm{~m}, 2 \mathrm{H})$, 7.81-7.83 (m, 2H), 8.30-8.32 (m, 2H).

\section{4-Benzoylbenzonitrile $(3 \mathrm{~m})^{3}$}<smiles>N#Cc1ccc(C(=O)c2ccccc2)cc1</smiles>

Purification: column chromatography (hexane/EtOAc $=20: 1, R_{\mathrm{f}}=0.33$ ). White solid. Yield was $85 \%$ (35.2 mg). $\quad{ }^{1} \mathrm{H}$ NMR (600 MHz, $\left.\mathrm{CDCl}_{3}\right): \delta 7.50-7.53(\mathrm{~m}, 2 \mathrm{H}), 7.62-7.65(\mathrm{~m}, 1 \mathrm{H}), 7.77-7.80(\mathrm{~m}, 4 \mathrm{H})$, 7.86-7.88 (m, 2H).

\section{H-fluorene-1-carbonitrile (3x) $)^{12}$}<smiles>N#Cc1cccc2c1Cc1ccccc1-2</smiles>

Purification: column chromatography (hexane/EtOAc $=20: 1, R_{\mathrm{f}}=0.45$ ). White solid. Yield was $87 \%$ (33.3 mg). $\quad{ }^{1} \mathrm{H}$ NMR (600 MHz, $\left.\mathrm{CDCl}_{3}\right): \delta 4.07$ (s, 2H), 7.39 (td, $\left.J=7.2,1.2 \mathrm{~Hz}, 1 \mathrm{H}\right), 7.43$ (t, $J=7.8$ $\mathrm{Hz}, 1 \mathrm{H}), 7.48(\mathrm{t}, J=7.8 \mathrm{~Hz}, 1 \mathrm{H}), 7.57(\mathrm{dd}, J=7.8,1.2 \mathrm{~Hz}, 1 \mathrm{H}), 7.61(\mathrm{~d}, J=7.2 \mathrm{~Hz}, 1 \mathrm{H}), 7.80(\mathrm{~d}, J=$ $7.8 \mathrm{~Hz}, 1 \mathrm{H}), 7.97(\mathrm{~d}, J=7.8 \mathrm{~Hz}, 1 \mathrm{H})$.

\section{Benzofuran-2-carbonitrile (3ab) ${ }^{8}$}


<smiles>N#Cc1cc2ccccc2o1</smiles>

Purification: column chromatography (hexane/EtOAc $=40: 1, R_{\mathrm{f}}=0.53$ ). Yellow oil. Yield was $96 \%$ (27.5 mg). ${ }^{1} \mathrm{H}$ NMR (600 MHz, $\left.\mathrm{CDCl}_{3}\right): \delta 7.37$ (ddd, $\left.J=8.4,7.1,1.2 \mathrm{~Hz}, 1 \mathrm{H}\right), 7.47$ (d, $J=0.9 \mathrm{~Hz}$, $1 \mathrm{H}), 7.51$ (ddd, $J=8.4,7.1,1.2 \mathrm{~Hz}, 1 \mathrm{H}), 7.56(\mathrm{dd}, J=8.4,1.2 \mathrm{~Hz}, 1 \mathrm{H}), 7.68(\mathrm{dd}, J=7.9,0.9 \mathrm{~Hz}, 1 \mathrm{H})$.

\section{(E)-2,3-Diphenylacrylonitrile (3ac) $)^{13}$}<smiles>N#C/C(=C/c1ccccc1)c1ccccc1</smiles>

Purification: column chromatography (hexane/EtOAc $=40: 1, R_{\mathrm{f}}=0.35$ ). Yellow solid. Yield was $80 \%(32.8 \mathrm{mg}) .(E / Z=96 / 4) \quad \boldsymbol{E}-3 \mathbf{a c}$ isomer: ${ }^{1} \mathrm{H}$ NMR $\left(600 \mathrm{MHz}, \mathrm{CDCl}_{3}\right): \delta 7.16-7.18(\mathrm{~m}, 2 \mathrm{H}), 7.22-$ $7.25(\mathrm{~m}, 2 \mathrm{H}), 7.28-7.31(\mathrm{~m}, 1 \mathrm{H}), 7.34-7.42(\mathrm{~m}, 6 \mathrm{H}) . \quad$ Z-3ac isomer: ${ }^{1} \mathrm{H}$ NMR $\left(600 \mathrm{MHz}, \mathrm{CDCl}_{3}\right): \delta$ 7.45-7.48 (m, 6H), 7.56 (s, 1H), 7.69-7.71 (m, 2H), 7.90-7.92 (m, 2H).

\section{4-Cyano- $N, N$-dipropylbenzenesulfonamide (3af) ${ }^{14}$}<smiles>CCCN(CCC)S(=O)(=O)c1ccc(C#N)cc1</smiles>

Purification: column chromatography (hexane/EtOAc $=10: 1, R_{\mathrm{f}}=0.32$ ). White solid. Yield was $92 \%$ (48.8 mg). $\quad{ }^{1} \mathrm{H}$ NMR (600 MHz, $\left.\mathrm{CDCl}_{3}\right): \delta 0.85$ (t, $\left.J=7.8 \mathrm{~Hz}, 6 \mathrm{H}\right), 1.50-1.56(\mathrm{~m}, 4 \mathrm{H}), 3.09$ (t, $J=7.8$ $\mathrm{Hz}, 4 \mathrm{H}), 7.78-7.80$ (m, 2H), 7.90-7.91 (m, 2H).

\section{4'-Methyl-[1,1'-biphenyl]-2-carbonitrile (3ag) $)^{15}$}<smiles>Cc1ccc(-c2ccccc2C#N)cc1</smiles>

Purification: column chromatography (hexane/EtOAc $=40: 1, R_{\mathrm{f}}=0.34$ ). Pale yellow solid. Yield was $98 \%(37.9 \mathrm{mg}) . \quad{ }^{1} \mathrm{H} \mathrm{NMR}\left(600 \mathrm{MHz}, \mathrm{CDCl}_{3}\right): \delta 2.43(\mathrm{~s}, 3 \mathrm{H}), 7.31$ (d, $\left.J=7.8 \mathrm{~Hz}, 2 \mathrm{H}\right), 7.42$ (td, $J$ 
$=7.8,1.2 \mathrm{~Hz}, 1 \mathrm{H}), 7.47(\mathrm{~d}, J=7.8 \mathrm{~Hz}, 2 \mathrm{H}), 7.51(\mathrm{dd}, J=7.8,1.2 \mathrm{~Hz}, 1 \mathrm{H}), 7.63(\mathrm{td}, J=7.8,1.2 \mathrm{~Hz}$, 1H), $7.76(\mathrm{dd}, J=7.8,1.2 \mathrm{~Hz}, 1 \mathrm{H})$. 


\section{Ni-Catalyzed Decarbonylative Cyanation of Estrone Derivatives}

Synthesis of Methyl 4-(((8R,9S,13S,14S)-13-Methyl-17-oxo-7,8,9,11,12,13,14,15,16,17decahydro-6H-cyclopenta[a]phenanthren-3-yl)oxy)methyl)benzoate (5)

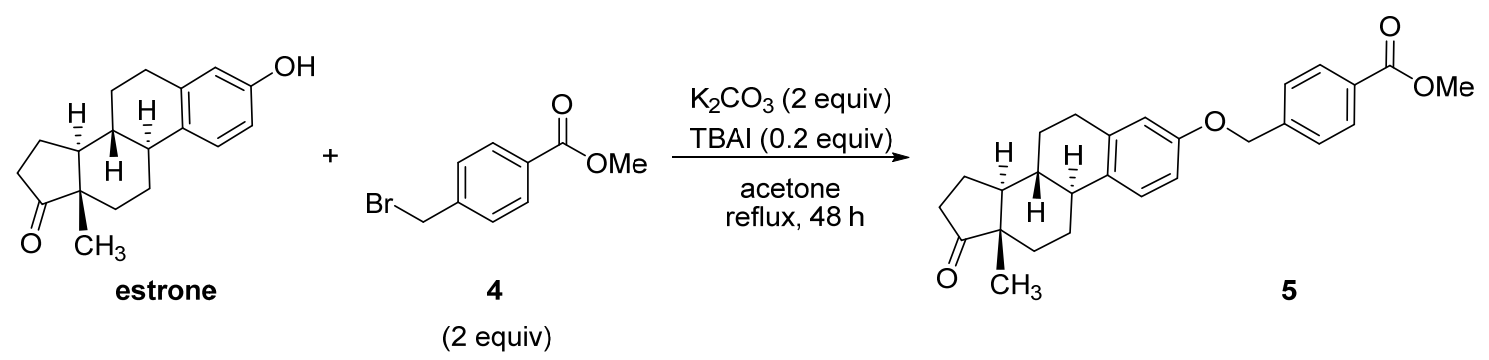

Compound 5 was synthesized according to a modified procedure. ${ }^{16}$ An oven-dried Schlenk tube containing a stirring bar was charged with estrone (270 mg, $1 \mathrm{mmol}$ ), compound 4 (458.2 mg, 2 mmol, 2 equiv), $\mathrm{K}_{2} \mathrm{CO}_{3}$ (276.4 mg, $2 \mathrm{mmol}, 2$ equiv), TBAI ( $73.9 \mathrm{mg}, 0.2 \mathrm{mmol}, 0.2$ equiv) and acetone (10 $\mathrm{mL}$ ). After the reaction mixture was heated to reflux for $48 \mathrm{~h}$, the solvent was removed under vacuum. The crude mixture was extracted with dichloromethane $(10 \mathrm{~mL} \times 3)$ and organic layers was combined, dried over $\mathrm{Na}_{2} \mathrm{SO}_{4}$, filtered, and the solvent removed under reduced pressure. The crude product was purified by column chromatography (hexane/EtOAc $=5: 1, R_{\mathrm{f}}=0.47$ ) to afford 5 quantitatively $(418 \mathrm{mg}$ ) as white solid. ${ }^{1} \mathrm{H}$ NMR (600 MHz, $\left.\mathrm{CDCl}_{3}\right): \delta 0.91(\mathrm{~s}, 3 \mathrm{H}), 1.39-1.65(\mathrm{~m}, 6 \mathrm{H}), 1.93-1.97(\mathrm{~m}, 1 \mathrm{H})$, $1.98-2.08(\mathrm{~m}, 2 \mathrm{H}), 2.14(\mathrm{dt}, J=19.0,8.8 \mathrm{~Hz}, 1 \mathrm{H}), 2.23-2.28(\mathrm{~m}, 1 \mathrm{H}), 2.37-2.41(\mathrm{~m}, 1 \mathrm{H}), 2.50(\mathrm{dd}, J=$ 19.0, $8.8 \mathrm{~Hz}, 1 \mathrm{H}), 2.88-2.91(\mathrm{~m}, 2 \mathrm{H}), 3.92(\mathrm{~s}, 3 \mathrm{H}), 5.10(\mathrm{~s}, 2 \mathrm{H}), 6.72(\mathrm{~d}, J=2.6 \mathrm{~Hz}, 1 \mathrm{H}), 6.77(\mathrm{dd}, J=$ 8.6, 3.0 Hz, 1H), 7.21 (d, $J=8.4 \mathrm{~Hz}, 1 \mathrm{H}), 7.50$ (d, $J=8.4 \mathrm{~Hz}, 2 \mathrm{H}), 8.05$ (dd, $J=7.2,1.8 \mathrm{~Hz}, 2 \mathrm{H})$; ${ }^{13} \mathrm{C}\left\{{ }^{1} \mathrm{H}\right\}$ NMR (151 MHz, $\left.\mathrm{CDCl}_{3}\right): \delta 14.0,21.7,26.0,26.6,29.8,31.7,36.0,38.4,44.1,48.1,50.5,52.3$, 69.4, 112.4, 115.0, 126.5, 127.0, 129.6, 130.0, 132.7, 138.0, 142.6, 156.6, 167.0, 221.1. FT-IR (KBr): 2934, 2910, 2859, 2830, 1734, 1719, 1605, 1499, 1454, 1437, 1414, 1279, 1254, 1236, 1175, 1109, 1036 $\mathrm{cm}^{-1}$. HRMS (FAB ${ }^{+}$): Calcd for $\mathrm{C}_{27} \mathrm{H}_{30} \mathrm{O}_{4}:$ 418.2144. Found: 418.2150. Anal. Calcd for $\mathrm{C}_{27} \mathrm{H}_{30} \mathrm{O}_{4}$ : C, 77.48; H, 7.23\%. Found: C, 77.41; H, 7.25\%.

Synthesis of 4-(((8R,9S,13S,14S)-13-Methyl-17-oxo-7,8,9,11,12,13,14,15,16,17-decahydro-6Hcyclopenta[a]phenanthren-3-yl)oxy)methyl)benzoic acid (6) ${ }^{17}$ 
(OMe

5
$\frac{\mathrm{LiOH} \text { (2 equiv) }}{\underset{\mathrm{THF} / \mathrm{H}_{2} \mathrm{O}}{\text { reflux }}}$

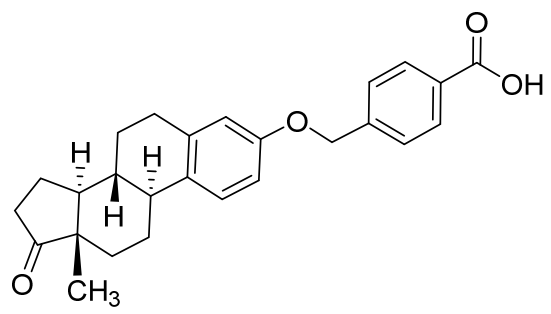

6

Compound 6 was subjected to hydrolysis according to the reported method. ${ }^{18}$ A solution of compound 5 (418 g, $1 \mathrm{mmol}$ ) and lithium hydroxide monohydrate ( $84 \mathrm{mg}, 2 \mathrm{mmol}$ ) in tetrahydrofuran $(2 \mathrm{~mL})$ and water $(2 \mathrm{~mL})$ was refluxed at $100{ }^{\circ} \mathrm{C}$. After $4 \mathrm{~h}$, the solvent was evaporated, and concentrated $\mathrm{HCl}$ was added to the residue. The precipitate was filtrated, washed with water, dried under vacuum to give a white solid (291 mg, 82\%). $\left.{ }^{1} \mathrm{H} \mathrm{NMR} \mathrm{(600} \mathrm{MHz,}\left(\mathrm{CD}_{3}\right)_{2} \mathrm{SO}\right): \delta 0.81(\mathrm{~s}, 3 \mathrm{H})$, $1.30-1.39$ (m, 3H), 1.44-1.57 (m, 3H), 1.72-1.77 (m, 1H), 1.89-1.96 (m, 2H), $2.05(\mathrm{dt}, J=18.5,8.5 \mathrm{~Hz}$, $1 \mathrm{H}), 2.14-2.18(\mathrm{~m}, 1 \mathrm{H}), 2.29-3.35(\mathrm{~m}, 1 \mathrm{H}), 2.43(\mathrm{dd}, J=18.5,8.5 \mathrm{~Hz}, 1 \mathrm{H}), 2.79-2.82(\mathrm{~m}, 2 \mathrm{H}), 3.36$ (brs, $1 \mathrm{H}), 5.14(\mathrm{~s}, 2 \mathrm{H}), 6.72(\mathrm{~d}, J=2.8 \mathrm{~Hz}, 1 \mathrm{H}), 6.76(\mathrm{dd}, J=8.6,2.8 \mathrm{~Hz}, 1 \mathrm{H}), 7.17(\mathrm{~d}, J=8.6 \mathrm{~Hz}, 1 \mathrm{H}), 7.52$ (d, $J=8.0 \mathrm{~Hz}, 2 \mathrm{H}), 7.95(\mathrm{~d}, J=8.0 \mathrm{~Hz}, 2 \mathrm{H}) ;{ }^{13} \mathrm{C}\left\{{ }^{1} \mathrm{H}\right\} \mathrm{NMR}\left(151 \mathrm{MHz},\left(\mathrm{CD}_{3}\right)_{2} \mathrm{SO}\right): \delta 13.6,21.2,25.5$, 26.1, 29.2, 31.4, 35.4, 37.8, 43.5, 47.4, 49.6, 68.4, 112.3, 114.6, 126.4, 127.2, 129.5, 130.3, 132.2, 137.6, 142.4, 156.0, 167.2, 219.8. FT-IR (KBr): 2932, 2876, 1734, 1678, 1611, 1497, 1425, 1254, 1159, 1057, $1005,860 \mathrm{~cm}^{-1}$. Anal. Calcd for $\mathrm{C}_{26} \mathrm{H}_{28} \mathrm{O}_{4}: \mathrm{C}, 77.20 ; \mathrm{H}, 6.98 \%$. Found: $\mathrm{C}, 77.03 ; \mathrm{H}, 6.80 \%$.

\section{4-((((8R,9S,13S,14S)-13-Methyl-17-ox0-7,8,9,11,12,13,14,15,16,17-decahydro-6H-cyclopenta[a]} phenanthren-3-yl)oxy)methyl)benzonitrile (3ah)

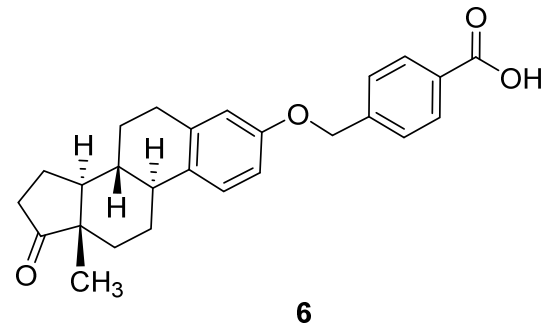

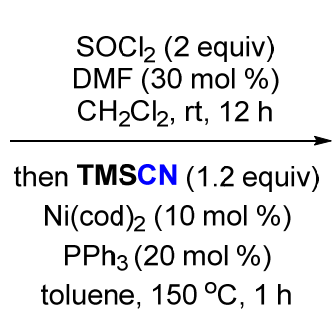

toluene, $150{ }^{\circ} \mathrm{C}, 1 \mathrm{~h}$

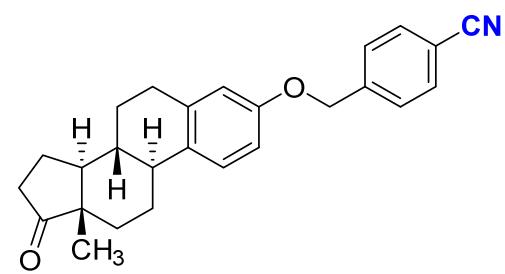

3ah

To the solution of anhydrous 1,2-dichloromethane $(0.8 \mathrm{~mL})$ and DMF $(5 \mu \mathrm{L})$ of carboxylic acid 6 (80.9 mg, $0.2 \mathrm{mmol}$ ) in an oven-dried Schlenk tube containing a stirring bar, thionyl chloride (47.6 mg, $0.4 \mathrm{mmol}, 2$ equiv) was added dropwise using a syringe. The reaction mixture was stirred at room temperature for $12 \mathrm{~h}$. The solvent and unreacted thionyl chloride were removed under reduced pressure. The resulting acyl chloride was used without additional purification. Then $\mathrm{Ni}(\operatorname{cod})_{2}(5.5$ 
$\mathrm{mg}, 0.02 \mathrm{mmol}, 10 \mathrm{~mol} \%), \mathrm{PPh}_{3}(10.5 \mathrm{mg}, 0.04 \mathrm{mmol}, 20.0 \mathrm{~mol} \%)$, toluene $(1 \mathrm{~mL})$, and TMSCN (23.4 $\mathrm{mg}, 0.24 \mathrm{mmol}, 1.2$ equiv) were successively added. The mixture was stirred at $150^{\circ} \mathrm{C}$ for $1 \mathrm{~h}$. After cooling to room temperature, the crude product was purified by column chromatography on silica gel (hexane/EtOAc $=5: 1, R_{\mathrm{f}}=0.39$ ) to afford the corresponding nitriles 3ah.

White solid. Yield was 74\% (57 mg). ${ }^{1} \mathrm{H}$ NMR (600 MHz, $\left.\mathrm{CDCl}_{3}\right): \delta 0.91(\mathrm{~s}, 3 \mathrm{H}), 1.40-1.65(\mathrm{~m}$, 6H), 1.94-1.97 (m, 1H), 1.99-2.08 (m, 2H), 2.15 (dt, $J=19.0,9.0 \mathrm{~Hz}, 1 \mathrm{H}), 2.23-2.29$ (m, 1H), 2.37-2.41 (m, 1H), $2.51(\mathrm{dd}, J=19.0,9.0 \mathrm{~Hz}, 1 \mathrm{H}), 2.88-2.91(\mathrm{~m}, 2 \mathrm{H}), 5.14(\mathrm{~s}, 2 \mathrm{H}), 6.71(\mathrm{~d}, J=3.0 \mathrm{~Hz}, 1 \mathrm{H}), 6.76$ $(\mathrm{dd}, J=8.4,3.0 \mathrm{~Hz}, 1 \mathrm{H}), 7.22(\mathrm{~d}, J=8.4 \mathrm{~Hz}, 1 \mathrm{H}), 7.57(\mathrm{~d}, J=8.4 \mathrm{~Hz}, 2 \mathrm{H}), 8.13(\mathrm{dt}, J=8.4,1.8 \mathrm{~Hz}$, $2 \mathrm{H}) ;{ }^{13} \mathrm{C}\left\{{ }^{1} \mathrm{H}\right\} \mathrm{NMR}\left(151 \mathrm{MHz}, \mathrm{CDCl}_{3}\right): \delta 14.0,21.7,26.0,26.6,29.8,31.7,36.0,38.4,44.1,48.1,50.5$, $68.9,112.4,115.0,126.7,127.3,131.9,132.7,133.0,138.2,145.7,156.3,168.2,221.1 . \quad$ FT-IR $(\mathrm{KBr})$ : 2991, 2926, 2378, 1734, 1606, 1219, 908, 793, $650 \mathrm{~cm}^{-1}$. Anal. Calcd for $\mathrm{C}_{26} \mathrm{H}_{27} \mathrm{NO}_{2}: \mathrm{C}, 81.01 ; \mathrm{H}$, 7.06; N, 3.63\%. Found: C, 81.10; H, 7.04; N, 3.58\%. 


\section{Mechanistic Studies}

6.1 A Stoichiometric Reaction of $\mathrm{Ni}(\mathrm{cod})_{2} / 2 \mathrm{PPh}_{3}$ with 1-Naphthoyl Chloride (1y)<smiles>O=C(Cl)c1cccc2ccccc12</smiles>

$1 y$

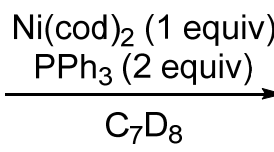

$\mathrm{rt}, 5 \mathrm{~min}$<smiles>O=C(c1cccc2ccccc12)[N+](P)(P)P(Cl)c1ccccc1</smiles>

7: $3 \%$<smiles>PP(Cl)(c1ccccc1)(c1ccccc1)c1cccc2ccccc12</smiles>

8: $64 \%$

$(0.05 \mathrm{mmol})$

An oven-dried $20 \mathrm{~mL}$ of Schlenk tube containing a stirring bar was charged with $\mathrm{Ni}(\mathrm{cod})_{2}(13.8 \mathrm{mg}$, $0.05 \mathrm{mmol}), \mathrm{PPh}_{3}(26.2 \mathrm{mg}, 0.1 \mathrm{mmol})$ in $\mathrm{C}_{7} \mathrm{D}_{8}(0.4 \mathrm{~mL})$ at room temperature. In a separate Schlenk tube, acyl chloride $1 \mathbf{y}(9.5 \mathrm{mg}, 0.05 \mathrm{mmol})$ was dissolved in $\mathrm{C}_{7} \mathrm{D}_{8}(0.2 \mathrm{~mL})$ at room temperature. The $\mathrm{Ni}(\operatorname{cod})_{2} / \mathrm{PPh}_{3}$ solution was transferred to the solution of $\mathbf{1 y}$ via a cannula. The resulting dark red solution was immediately subjected to the ${ }^{31} \mathrm{P}\left\{{ }^{1} \mathrm{H}\right\} \mathrm{NMR}$ measurements.

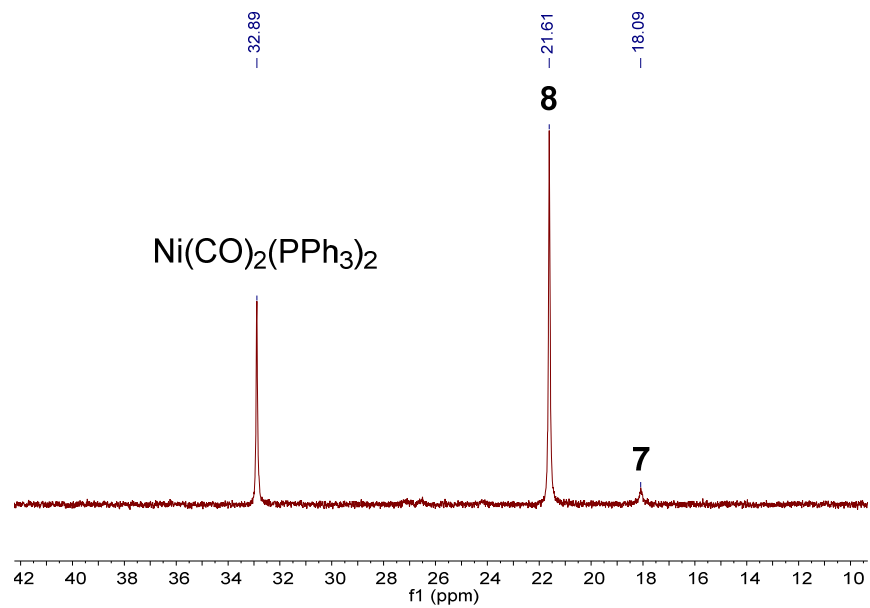

Figure S1. ${ }^{31} \mathrm{P}\left\{{ }^{1} \mathrm{H}\right\} \mathrm{NMR}$ spectrum of a stoichiometric reaction of $\mathrm{Ni}(\mathrm{cod})_{2}$ and $\mathrm{PPh}_{3}$ with $\mathbf{1 y}$ at $\mathrm{rt}$ in $\mathrm{C}_{7} \mathrm{D}_{8}$ after 5 min. 


\subsection{Reaction of $\mathrm{Ni}(\mathrm{cod})_{2} / 2 \mathrm{PPh}_{3}$ with $1 \mathrm{y}$ in the Presence of TMSCN}

Characterization of Ni complexes. To an oven-dried $20 \mathrm{~mL}$ of Schlenk tube with a stirring bar, were added $\mathrm{Ni}(\mathrm{cod})_{2}(13.8 \mathrm{mg}, 0.05 \mathrm{mmol}), \mathrm{PPh}_{3}(26.2 \mathrm{mg}, 0.1 \mathrm{mmol})$, and $\mathrm{C}_{7} \mathrm{D}_{8}(0.4 \mathrm{~mL})$ under an argon atmosphere. In the other Schlenk tube, 1-naphthoyl chloride (1y) (9.5 mg, $0.05 \mathrm{mmol})$ and TMSCN (6.0 mg, $0.06 \mathrm{mmol}, 1.2$ equiv) were dissolved in $\mathrm{C}_{7} \mathrm{D}_{8}(0.2 \mathrm{~mL})$. The $\mathrm{Ni}(\mathrm{cod})_{2} / \mathrm{PPh}_{3}$ solution was transferred to an NMR tube under Ar. After the solution of 1y and TMSCN was added to the NMR tube, the reaction was immediately monitored with ${ }^{31} \mathrm{P}\left\{{ }^{1} \mathrm{H}\right\}$ NMR spectroscopy at room temperature.<smiles>O=C(Cl)c1cccc2ccccc12</smiles>

$1 y$ $(0.05 \mathrm{mmol})$

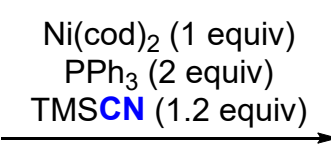

$\mathrm{C}_{7} \mathrm{D}_{8}, \mathrm{rt}$ unidentified complex

A

${ }^{31} \mathrm{P}\left\{{ }^{1} \mathrm{H}\right\}$ NMR: $33.6 \mathrm{ppm}$

${ }^{31} \mathrm{P}\left\{{ }^{1} \mathrm{H}\right\}$ NMR: 32.9 ppm

$\mathrm{Ni}(\mathrm{CO})_{2}\left(\mathrm{PPh}_{3}\right)_{2}$

B

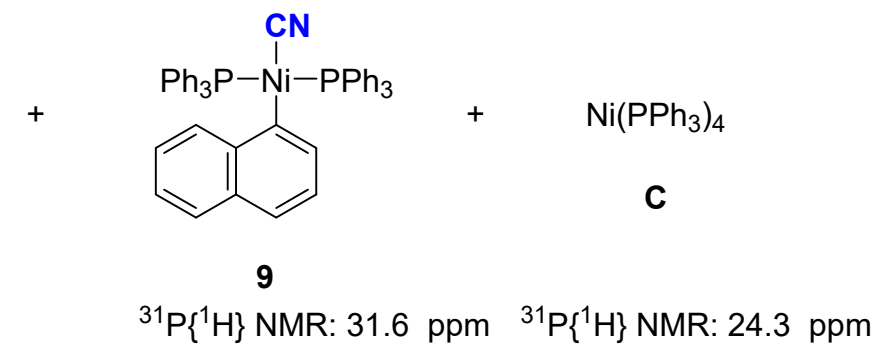

Table S11

\begin{tabular}{cccccc}
\hline Entry & Time $(\min )$ & $\mathbf{A}(\%)$ & $\mathbf{B}(\%)$ & $\mathbf{9}(\%)$ & $\mathbf{C}(\%)$ \\
\hline $\mathbf{1}$ & $\mathbf{5}$ & $\mathbf{2}$ & $\mathbf{2 8}$ & $\mathbf{3 6}$ & $\mathbf{4}$ \\
2 & 8 & 2 & 28 & 36 & 4 \\
3 & 12 & 3 & 39 & 35 & 4 \\
4 & 16 & 3 & 39 & 39 & 4 \\
5 & 23 & 3 & 38 & 34 & 4 \\
6 & 30 & 3 & 40 & 35 & 4 \\
7 & 60 & 3 & 38 & 33 & 0 \\
\hline
\end{tabular}


Determination of the ${ }^{31} \mathbf{P}\left\{{ }^{1} \mathbf{H}\right\}$ NMR yield. A sealed capillary containing a deuterium toluene solution of $\mathrm{PPh}_{3}(13.1 \mathrm{mg}, 0.05 \mathrm{mmol})$ was put into an oven dried NMR tube. The NMR tube was moved to a big Schlenk tube and vacuum, then argon refilled three times. To the NMR tube charged with $\mathrm{Ni}(\mathrm{cod})_{2}(13.8 \mathrm{mg}, 0.05 \mathrm{mmol}), \mathrm{PPh}_{3}(26.2 \mathrm{mg}, 0.1 \mathrm{mmol}, 2$ equiv), $3 \mathbf{y}(7.7 \mathrm{mg}, 0.05 \mathrm{mmol})$, TMSCN (6.0 mg, $0.06 \mathrm{mmol}, 1.2$ equiv) and $\mathrm{C}_{7} \mathrm{D}_{8}(0.5 \mathrm{~mL})$. The NMR tube was capped and the ${ }^{31} \mathrm{P}\left\{{ }^{1} \mathrm{H}\right\}$ NMR at room temperature was measured.

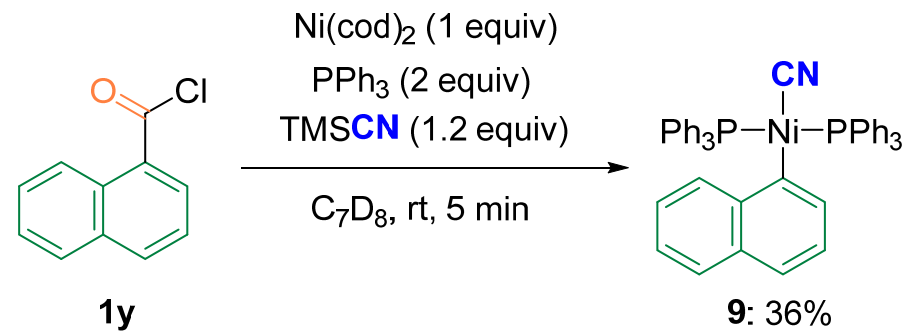

$(0.05 \mathrm{mmol})$

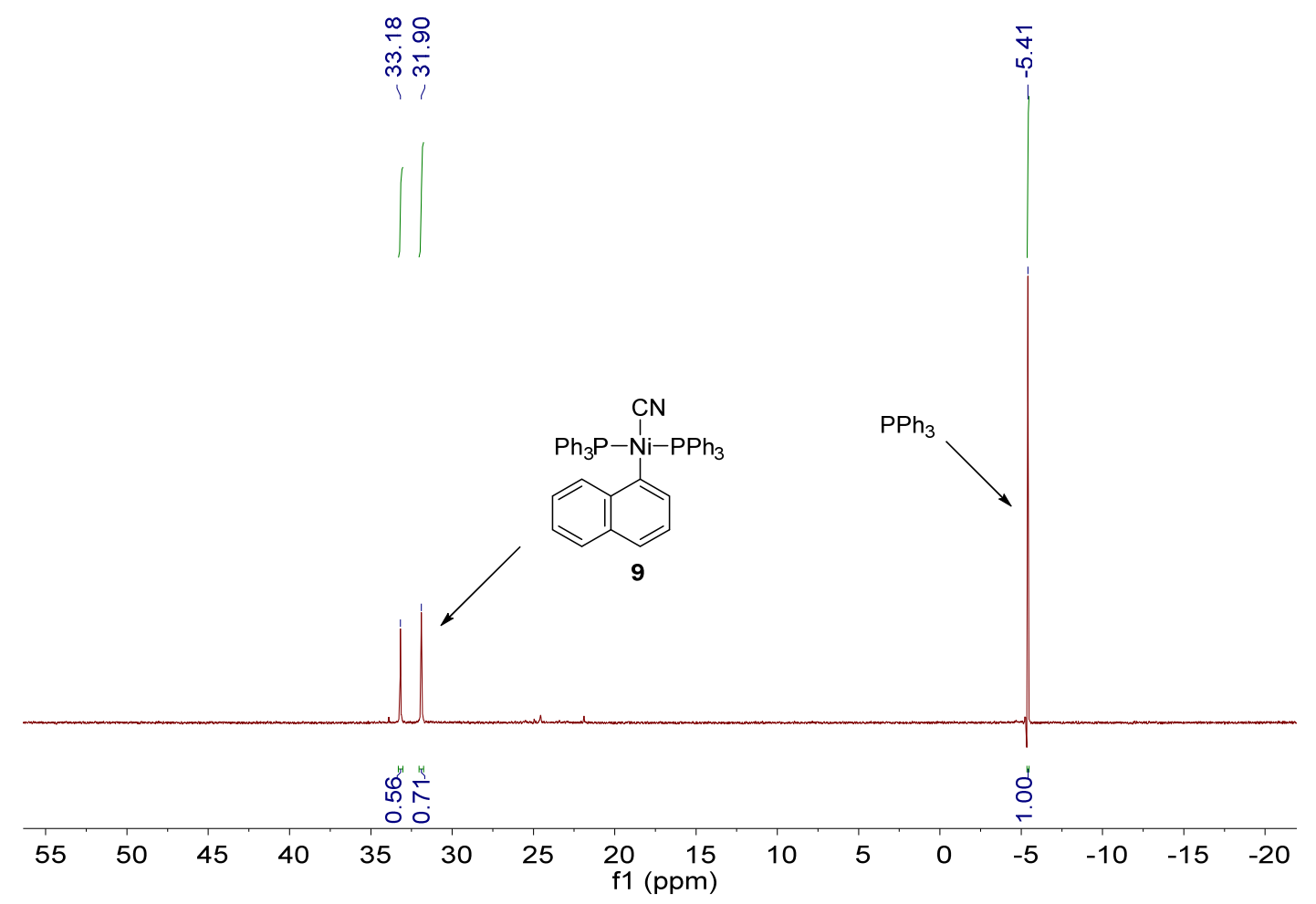


Characterization of Reductive Elimination Product 3y. To an oven-dried $20 \mathrm{~mL}$ of Schlenk tube with a stirring bar, were added $\mathrm{Ni}(\mathrm{cod})_{2}(13.8 \mathrm{mg}, 0.05 \mathrm{mmol}), \mathrm{PPh}_{3}(26.2 \mathrm{mg}, 0.1 \mathrm{mmol})$, and toluene $(0.4 \mathrm{~mL})$ under an argon atmosphere. In the other Schlenk tube, 1-naphthoyl chloride (1y) (9.5 mg, $0.05 \mathrm{mmol})$, TMSCN (6.0 mg, $0.06 \mathrm{mmol}, 1.2$ equiv) and $n$-dodecane ( $8.5 \mathrm{mg}, 0.05 \mathrm{mmol})$ was dissolved in toluene $(0.2 \mathrm{~mL})$. The $\mathrm{Ni}(\mathrm{cod})_{2} / \mathrm{PPh}_{3}$ solution was transferred into the solution containing 1y and TMSCN via a cannula. The reaction was monitored by GC.

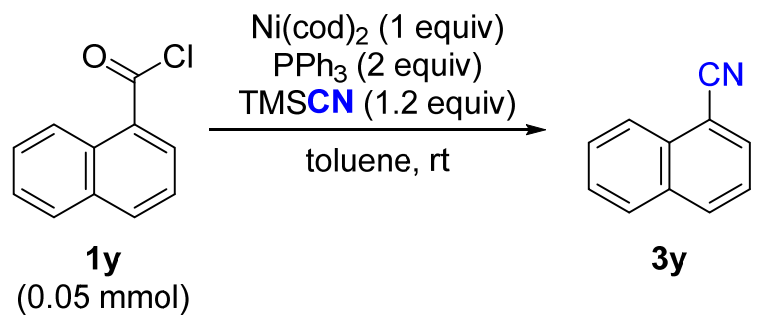

Table S12

\begin{tabular}{ccc}
\hline Entry & Time $(\mathrm{h})$ & GC yield of $\mathbf{3 y}(\%)$ \\
\hline $\mathbf{1}$ & $\mathbf{0 . 0 8}(\mathbf{=} \mathbf{5} \mathbf{~ m i n})$ & $\mathbf{4 7}$ \\
2 & 0.5 & 58 \\
4 & 1 & 60 \\
5 & 3 & 60 \\
6 & 6 & 60 \\
\hline
\end{tabular}




\subsection{Synthesis of trans-Ni(1-Np)Cl( $\left(\mathrm{PPh}_{3}\right)_{2}(8)$}

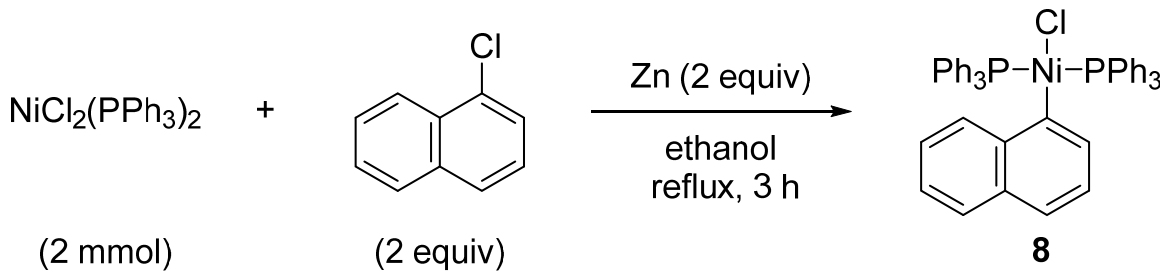

trans $-\mathrm{Ni}(1-\mathrm{Np}) \mathrm{Cl}\left(\mathrm{PPh}_{3}\right)_{2}(\mathbf{8})$ was prepared according to a modified literature procedure. ${ }^{19}$ To the ethanol $(12 \mathrm{~mL})$ solution of $\mathrm{NiCl}_{2}\left(\mathrm{PPh}_{3}\right)_{2}(1.3 \mathrm{~g}, 2 \mathrm{mmol})$ in an oven-dried Schlenk tube with a stirring bar were added 1-chloronaphthalene (650 mg, $0.54 \mathrm{~mL}, 4 \mathrm{mmol}), \mathrm{Zn}$ dust (262 mg, $4 \mathrm{mmol})$. The reaction mixture was stirred under reflux for $3 \mathrm{~h}$ and allowed to cool to room temperature. The yellow solid in green solution was filtrated, washed with ethanol $(20 \mathrm{~mL})$, dissolved in hot 1,2-dichloroethane $(10 \mathrm{~mL})$, and filtered through a pad of Celite. The Celite was then washed again with hot 1,2dichloroethane $(20 \mathrm{~mL})$. Evaporation of the combined filtrates and orange oil was washed with hexane $(60 \mathrm{~mL})$, which was kept at $-32{ }^{\circ} \mathrm{C}$ overnight. The resulting orange solid was separated by filtration, washed with hexane $(2 \times 20 \mathrm{~mL})$, and dried under vacuum to yield trans-Ni(1-Np)Cl( $\left.\mathrm{PPh}_{3}\right)_{2}(8)$ in $74 \%$ yield $(1.1 \mathrm{~g}, 1.48 \mathrm{mmol}) . \quad{ }^{1} \mathrm{H} \mathrm{NMR}\left(600 \mathrm{MHz}, \mathrm{CD}_{2} \mathrm{Cl}_{2}\right): \delta 6.45(\mathrm{~s}, 1 \mathrm{H}), 6.72(\mathrm{~d}, J=7.9 \mathrm{~Hz}, 1 \mathrm{H}), 6.95$ $(\mathrm{m}, J=7.7 \mathrm{~Hz}, 1 \mathrm{H}), 7.01-7.06(\mathrm{~m}, 2 \mathrm{H}), 7.12-7.18(\mathrm{~m}, J=7.8 \mathrm{~Hz}, 13 \mathrm{H}), 7.25(\mathrm{~s}, 6 \mathrm{H}), 7.40-7.53(\mathrm{~m}$, $12 \mathrm{H}), 9.14(\mathrm{~d}, J=8.1 \mathrm{~Hz}, 1 \mathrm{H}) ;{ }^{31} \mathrm{P}\left\{{ }^{1} \mathrm{H}\right\} \mathrm{NMR}\left(243 \mathrm{MHz}, \mathrm{C}_{6} \mathrm{D}_{6}\right): \delta 21.6$. 


\subsection{Reaction of Complex 8 with TMSCN}

To an NMR tube charged with 8 (37.3 mg, $0.05 \mathrm{mmol})$, were added $\mathrm{C}_{7} \mathrm{D}_{8}(0.6 \mathrm{~mL})$, TMSCN (6.0 mg, $0.06 \mathrm{mmol}, 1.2$ equiv), and $n$-dodecane $(8.5 \mathrm{mg}, 0.05 \mathrm{mmol})$ as an internal standard under an argon atmosphere. The mixture was monitored by the ${ }^{31} \mathrm{P}\left\{{ }^{1} \mathrm{H}\right\}$ NMR spectroscopy at room temperature, showing the formation of complex 9 in $78 \%$ yield. Next, the NMR tube was put in an oil bath at $80^{\circ} \mathrm{C}$ for $1 \mathrm{~h}$. The crude mixture was measured by GC, indicating the formation of $\mathbf{3 y}$ in $76 \% \mathrm{GC}$ yield.

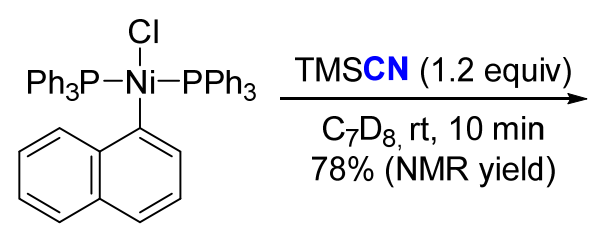

8<smiles>N#CC(P)(c1cccc2ccccc12)[P+]([PH])(C#N)c1ccccc1</smiles>

9 $80^{\circ} \mathrm{C}, 1 \mathrm{~h}$<smiles>N#Cc1cccc2ccccc12</smiles>

3y: $76 \%$ (GC yield) 


\subsection{Formation of $\mathrm{Ni}(1-\mathrm{NpCO}) \mathrm{Cl}\left(\mathrm{PEt}_{3}\right)_{2}(10)$ and Its Decarbonylation}

\subsubsection{Decarbonylation of 10 at Room Temperature}

To an oven-dried $20 \mathrm{~mL}$ of Schlenk tube with a stirring bar, were added $\mathrm{Ni}(\mathrm{cod})_{2}(13.8 \mathrm{mg}, 0.05$ mmol), $\mathrm{PEt}_{3}(11.8 \mathrm{mg}, 0.1 \mathrm{mmol})$, and $\mathrm{C}_{7} \mathrm{D}_{8}(0.4 \mathrm{~mL})$ under an argon atmosphere. In the other Schlenk tube, 1-naphthoyl chloride (1y) $(9.5 \mathrm{mg}, 0.05 \mathrm{mmol})$ was dissolved in $\mathrm{C}_{7} \mathrm{D}_{8}(0.2 \mathrm{~mL})$. The $\mathrm{Ni}(\mathrm{cod})_{2} / \mathrm{PEt}_{3}$ solution was transferred into the solution of $\mathbf{1 y}$ via a cannula. The resulting dark red solution was immediately transferred to an NMR tube under Ar. The reaction was monitored with ${ }^{31} \mathrm{P}\left\{{ }^{1} \mathrm{H}\right\}$ NMR spectroscopy at room temperature.

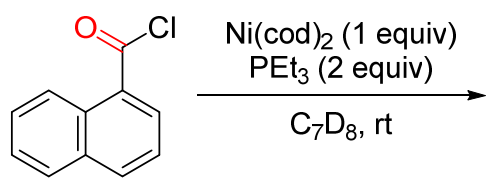

$1 y$ (0.05 mmol)

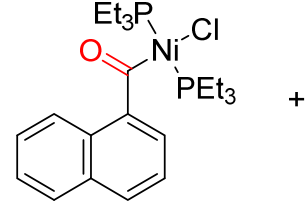

10<smiles></smiles>

11

${ }^{31} \mathrm{P}\left\{{ }^{1} \mathrm{H}\right\}$ NMR: $8.9 \mathrm{ppm} \quad{ }^{31} \mathrm{P}\left\{{ }^{1} \mathrm{H}\right\}$ NMR: $10.9 \mathrm{ppm}$

$5 \min \quad 91$




\subsubsection{Decarbonylation of 10 at $50{ }^{\circ} \mathrm{C}$}

To an oven-dried Schlenk tube with a stirring bar, were added Ni(cod) $2(13.8 \mathrm{mg}, 0.05 \mathrm{mmol}), \mathrm{PEt}_{3}$ (11.8 $\mathrm{mg}, 0.1 \mathrm{mmol})$, and $\mathrm{C}_{7} \mathrm{D}_{8}(0.4 \mathrm{~mL})$ under an argon atmosphere. In the other Schlenk tube, 1naphthoyl chloride (1y) $(9.5 \mathrm{mg}, 0.05 \mathrm{mmol})$ was dissolved in $\mathrm{C}_{7} \mathrm{D}_{8}(0.2 \mathrm{~mL})$. The Ni(cod) $2 / \mathrm{PEt}_{3}$ solution was transferred into the solution of $\mathbf{1 y}$ via a cannula. The resulting dark red solution was immediately transferred to the NMR tube under Ar. The NMR tube was monitored by ${ }^{31} \mathrm{P}\left\{{ }^{1} \mathrm{H}\right\} \mathrm{NMR}$ spectroscopy at $50{ }^{\circ} \mathrm{C}$.

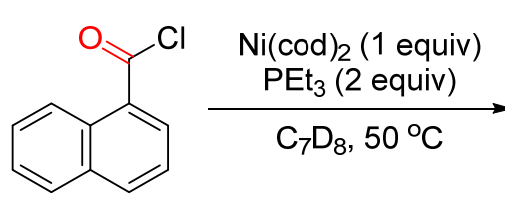

$1 y$ $(0.05 \mathrm{mmol})$

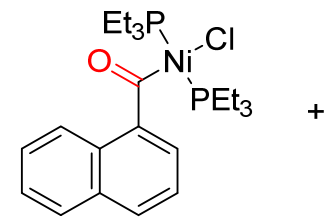

10<smiles>CCN(Cl)c1cccc2ccccc12</smiles>

11

${ }^{31} \mathrm{P}\left\{{ }^{1} \mathrm{H}\right\}$ NMR: $8.9 \mathrm{ppm} \quad{ }^{31} \mathrm{P}\left\{{ }^{1} \mathrm{H}\right\}$ NMR: $10.9 \mathrm{ppm}$

\section{Table S13}

\begin{tabular}{cccc}
\hline Entry & Time $(\mathrm{h})$ & $\mathbf{1 0}(\%)$ & $\mathbf{1 1}(\%)$ \\
\hline 1 & $0.08(=5 \mathrm{~min})$ & 75 & 19 \\
2 & $0.5(=30 \mathrm{~min})$ & 69 & 25 \\
3 & 3 & 63 & 30 \\
4 & 5 & 56 & 37 \\
5 & 24 & 56 & 38 \\
\hline
\end{tabular}




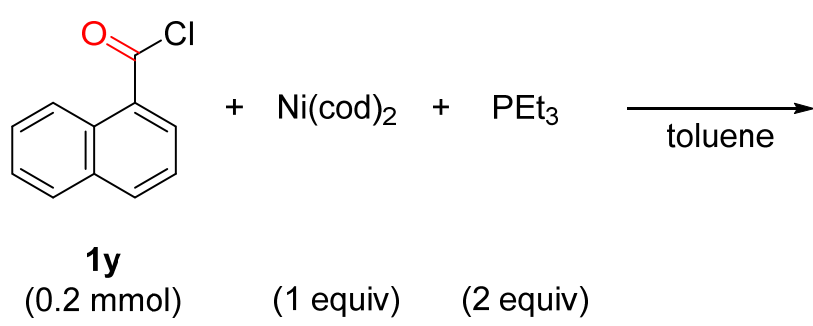<smiles>CCP[N+](CC)(C(=O)c1cccc2ccccc12)[PH](Cl)(Cl)CC</smiles>

10

To an oven-dried $20 \mathrm{~mL}$ of Schlenk tube containing a stirring bar, were added $\mathrm{Ni}(\mathrm{cod})_{2}(55 \mathrm{mg}, 0.2$ $\mathrm{mmol}), \mathrm{PEt}_{3}(47.3 \mathrm{mg}, 0.4 \mathrm{mmol})$, and toluene $(0.4 \mathrm{~mL})$ under an argon atmosphere. In the other Schlenk tube, 1-naphthoyl chloride $\mathbf{( 1 y )}(38.1 \mathrm{mg}, 0.2 \mathrm{mmol})$ was dissolved in toluene $(0.2 \mathrm{~mL})$. The $\mathrm{Ni}(\mathrm{cod})_{2} / \mathrm{PEt}_{3}$ solution was transferred to the solution of $\mathbf{1 y}$ via a cannula. The resulting dark red solution was stirred for $5 \mathrm{~min}$ and transferred to an NMR tube. Anhydrous hexane $(1 \mathrm{~mL})$ was added to the NMR tube, and the tube was sealed and placed into a $-20^{\circ} \mathrm{C}$ freezer overnight to afford red crystals of 10 in $45 \%$ yield (44 mg, $0.09 \mathrm{mmol}) . \quad{ }^{1} \mathrm{H} \mathrm{NMR}\left(600 \mathrm{MHz}, \mathrm{CD}_{2} \mathrm{Cl}_{2}\right): \delta 1.12\left(\mathrm{dt}, J_{\mathrm{P}-\mathrm{H}}=15.0 \mathrm{~Hz}, J=\right.$ $7.2 \mathrm{~Hz}, 18 \mathrm{H}), 1.51\left(\mathrm{~d}, J_{\mathrm{P}-\mathrm{H}}=114 \mathrm{~Hz}, 12 \mathrm{H}\right), 7.49(\mathrm{t}, J=7.5 \mathrm{~Hz}, 1 \mathrm{H}), 7.58(\mathrm{t}, J=7.5 \mathrm{~Hz}, 1 \mathrm{H}), 7.73(\mathrm{t}, J$ $=7.5 \mathrm{~Hz}, 1 \mathrm{H}), 7.85(\mathrm{~d}, J=8.1 \mathrm{~Hz}, 1 \mathrm{H}), 7.97(\mathrm{~d}, J=8.1 \mathrm{~Hz}, 1 \mathrm{H}), 9.27$ (brs, $1 \mathrm{H}), 9.97(\mathrm{brs}, 1 \mathrm{H}) ;{ }^{13} \mathrm{C}\left\{{ }^{1} \mathrm{H}\right\}$ NMR (151 MHz, $\mathrm{CD}_{2} \mathrm{Cl}_{2}$ ): $\delta$ 7.6, 14.0 (apparent triplet due to virtual coupling, ${ }^{1+3} \mathrm{JP}_{\mathrm{P}-\mathrm{C}}=13 \mathrm{~Hz}$ ), 123.2 , 124.6, 125.5, 126.0, 127.7, 127.9, 128.3, 131.8, 133.2, 133.5, $136.3\left(\mathrm{t}, J_{\mathrm{P}-\mathrm{C}}=5 \mathrm{~Hz}\right) ;{ }^{31} \mathrm{P}\left\{{ }^{1} \mathrm{H}\right\} \mathrm{NMR}(243$ $\mathrm{MHz}, \mathrm{CD}_{2} \mathrm{Cl}_{2}$ ): $\delta$ 8.7. FT-IR (KBr): 2964, 2931, 2904, 2873, 1634, 1601, 1504, 1454, 1416,1375, 1339, 1254, 1196, 1155, 885, 820, 787, 762, 745, 721, 698, 678, $658 \mathrm{~cm}^{-1}$. Anal. Calcd for $\mathrm{C}_{23} \mathrm{H}_{37} \mathrm{ClOP}_{2} \mathrm{Ni}: \mathrm{C}, 56.88 ; \mathrm{H}, 7.68 \%$. Found: C, 56.85; H, 7.81\%. 


\subsection{Synthesis of trans-Ni(1-Np)Cl( $\left.\mathrm{PEt}_{3}\right)_{2}(11)$}

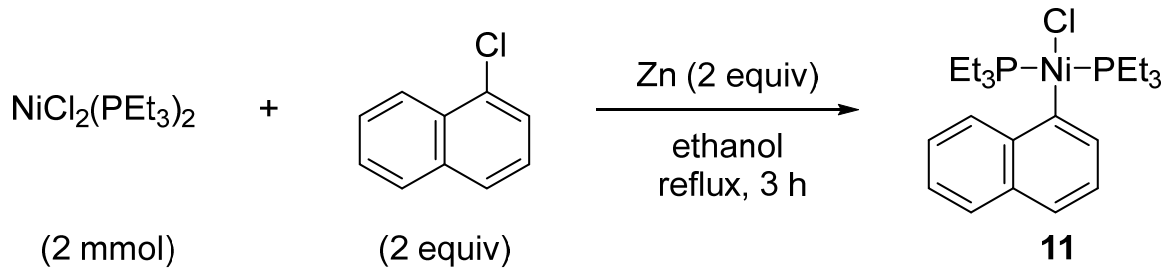

trans $-\mathrm{Ni}(1-\mathrm{Np}) \mathrm{Cl}\left(\mathrm{PEt}_{3}\right)_{2}(\mathbf{1 1})$ was prepared according to a modified procedure. ${ }^{18}$ To the ethanol (12 $\mathrm{mL}$ ) solution of $\mathrm{NiCl}_{2}\left(\mathrm{PEt}_{3}\right)_{2}$ (732 mg, $\left.2 \mathrm{mmol}\right)$, 1-chloronaphthalene (650 mg, $\left.0.54 \mathrm{~mL}, 4 \mathrm{mmol}\right)$ in an oven-dried $50 \mathrm{~mL}$ of Schlenk tube with a stirring bar, was added $\mathrm{Zn}$ dust (262 mg, $4 \mathrm{mmol}$ ). The reaction mixture was stirred under reflux for $3 \mathrm{~h}$ and allowed to cool to room temperature. The yellow solid was separated, washed with ethanol $(20 \mathrm{~mL})$, dissolved in hot 1,2-dichloroethane $(10 \mathrm{~mL})$, and filtered through a pad of Celite. The Celite was then washed again with additional 1,2-dichloroethane $(20 \mathrm{~mL})$. Evaporation of the combined filtrates and the oily orange solid was washed with hexane (60 $\mathrm{mL}$ ), which was kept at $-32{ }^{\circ} \mathrm{C}$ overnight. The resulting orange solid was separated by filtration, washed with hexane $(2 \times 20 \mathrm{~mL})$, and dried under vacuum to yield trans-Ni(1-Np)Cl( $\left.\mathrm{PEt}_{3}\right)_{2}(\mathbf{1 1})$ in $50 \%$ yield $(458 \mathrm{mg}, 1 \mathrm{mmol}) . \quad{ }^{1} \mathrm{H} \mathrm{NMR}\left(600 \mathrm{MHz},\left(\mathrm{CD}_{3}\right)_{2} \mathrm{CO}\right): \delta 1.06\left(\mathrm{dt}, J_{\mathrm{P}-\mathrm{H}}=15.6 \mathrm{~Hz}, J=7.2 \mathrm{~Hz}, 18 \mathrm{H}\right)$, $1.24\left(\mathrm{brd}, J_{\mathrm{P}-\mathrm{H}}=72.6 \mathrm{~Hz}, 12 \mathrm{H}\right), 7.06(\mathrm{t}, J=7.5 \mathrm{~Hz}, 1 \mathrm{H}), 7.26(\mathrm{~d}, J=8.2 \mathrm{~Hz}, 1 \mathrm{H}), 7.36(\mathrm{t}, J=7.5 \mathrm{~Hz}$, $1 \mathrm{H}), 7.46(\mathrm{t}, J=7.5 \mathrm{~Hz}, 1 \mathrm{H}), 7.52(\mathrm{~d}, J=7.0 \mathrm{~Hz}, 1 \mathrm{H}), 7.63(\mathrm{~d}, J=8.2 \mathrm{~Hz}, 1 \mathrm{H}), 9.23(\mathrm{~d}, J=8.2 \mathrm{~Hz}, 1 \mathrm{H})$; ${ }^{13} \mathrm{C}\left\{{ }^{1} \mathrm{H}\right\}$ NMR $\left(151 \mathrm{MHz},\left(\mathrm{CD}_{3}\right)_{2} \mathrm{CO}\right): \delta 8.3,14.3$ (apparent triplet due to virtual coupling, ${ }^{1+3} J_{\mathrm{P}-\mathrm{C}}=13$ $\mathrm{Hz}), 121.9,123.9,125.1,125.6,129.0,133.8,133.9,134.3,140.9,157.5\left(\mathrm{t}, J_{\mathrm{P}-\mathrm{C}}=34.2 \mathrm{~Hz}\right) ;{ }^{31} \mathrm{P}\left\{{ }^{1} \mathrm{H}\right\}$ NMR (243 MHz, $\left.\mathrm{C}_{6} \mathrm{D}_{6}\right) \delta$ 10.9. FT-IR (KBr): 3040, 2963, 2930, 2909, 2872, 1541, 1491, 1452, 1412, 1369, 1240, 1032, 1009, 1001, 953, 789, 760, $721 \mathrm{~cm}^{-1}$. Anal. Calcd for $\mathrm{C}_{22} \mathrm{H}_{37} \mathrm{ClP}_{2} \mathrm{Ni}$ : C, 57.74; $\mathrm{H}$, $8.15 \%$. Found: C, $57.35 ; \mathrm{H}, 8.40 \%$. 


\subsection{Reaction of $1 \mathrm{y}$ with $\mathrm{Ni}(\operatorname{cod})_{2} / 2 \mathrm{PEt}_{3}$ in the Presence of TMSCN}

To an oven-dried Schlenk tube with a stirring bar, were added Ni(cod) $2(13.8 \mathrm{mg}, 0.05 \mathrm{mmol}), \mathrm{PEt}_{3}$ (11.8 $\mathrm{mg}, 0.1 \mathrm{mmol})$, and $\mathrm{C}_{7} \mathrm{D}_{8}(0.4 \mathrm{~mL})$ under an argon atmosphere. In the other Schlenk tube, 1naphthoyl chloride $(\mathbf{1 y})(9.5 \mathrm{mg}, 0.05 \mathrm{mmol})$ was dissolved in $\mathrm{C}_{7} \mathrm{D}_{8}(0.2 \mathrm{~mL})$. The Ni(cod) $)_{2} / \mathrm{PEt}_{3}$ solution was transferred into the solution of $\mathbf{1 y}$ via a cannula. After $5 \mathrm{~min}$, TMSCN $(6.0 \mathrm{mg}, 0.06$ mmol, 1.2 equiv) was added to the mixture at room temperature. The resulting orange solution was immediately transferred to an NMR tube under Ar. The reaction was monitored by the ${ }^{31} \mathrm{P}\left\{{ }^{1} \mathrm{H}\right\} \mathrm{NMR}$ spectroscopy at room temperature.<smiles>O=C(Cl)c1cccc2ccccc12</smiles>

$1 \mathrm{y}$

$(0.05 \mathrm{mmol})$
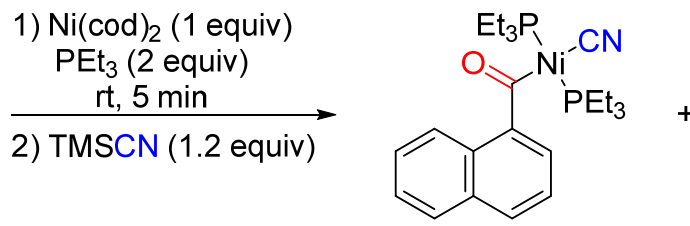

12

${ }^{31} \mathrm{P}\left\{{ }^{1} \mathrm{H}\right\}$ NMR: $15.1 \mathrm{ppm} \quad{ }^{31} \mathrm{P}\left\{{ }^{1} \mathrm{H}\right\}$ NMR: $18.6 \mathrm{ppm}$<smiles>CCP(C)(C)(CC)c1cccc2ccccc12</smiles>

13
Table S14

\begin{tabular}{cccc}
\hline Entry & Time $(\min )$ & $\mathbf{1 2}(\%)$ & $\mathbf{1 3}(\%)$ \\
\hline 1 & 2 & 66 & 11 \\
2 & 7 & 56 & 11 \\
3 & 30 & 41 & 12 \\
4 & 62 & 31 & 16 \\
5 & 92 & 27 & 22 \\
6 & 135 & 25 & 25 \\
7 & 150 & 21 & 26 \\
8 & 210 & 0 & 27 \\
\hline
\end{tabular}




\subsection{Synthesis of trans-Ni(1-Np)CN(PEt $)_{2}(13)$ by Oxidative Addition of $3 y$ to $\mathrm{Ni}(0)$ via C-CN Bond}

\section{Cleavage}<smiles>N#Cc1cccc2ccccc12</smiles>

$3 y$

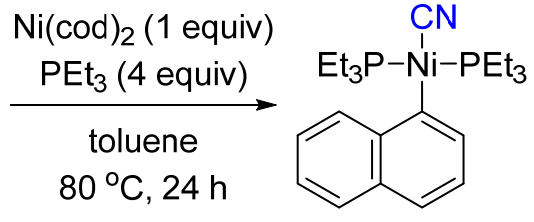

13

To the toluene $(1 \mathrm{~mL})$ solution of $\mathrm{Ni}(\operatorname{cod})_{2}(200 \mathrm{mg}, 0.72 \mathrm{mmol})$ in an oven-dried $50 \mathrm{~mL}$ of Schlenk tube with a stirring bar, was added $\mathrm{PEt}_{3}(340 \mathrm{mg}, 2.88 \mathrm{mmol}, 4$ equiv) under argon atmosphere. After the reaction mixture was stirred for $1 \mathrm{~min}, \mathbf{3 y}(110.2 \mathrm{mg}, 0.72 \mathrm{mmol})$ was added to the reaction mixture, which was stirred at $80{ }^{\circ} \mathrm{C}$ for $24 \mathrm{~h}$ and allowed to cool to room temperature. The reaction mixture was filtered through a pad of Celite. The Celite was then washed with 1,2-dichloroethane $(20 \mathrm{~mL})$. Evaporation of the filtrates and a crude product was washed with hexane $(20 \mathrm{~mL})$. Yellow precipitates were formed and separated through a membrane filter. The yellow solid was washed with hexane $(2$ $\times 20 \mathrm{~mL})$ and dried under vacuum to give trans $-\mathrm{Ni}(1-\mathrm{Np}) \mathrm{CN}\left(\mathrm{PEt}_{3}\right)_{2}(\mathbf{1 3})$ in $72 \%$ yield $(232.9 \mathrm{mg}, 0.52$ mmol). Single crystals of complex 13 were obtained by diffusion method of the $\mathrm{CH}_{2} \mathrm{Cl}_{2}$ solution containing 13 with hexane at room temperature. ${ }^{1} \mathrm{H}$ NMR $\left(600 \mathrm{MHz}, \mathrm{CD}_{2} \mathrm{Cl}_{2}\right): \delta 1.06\left(\mathrm{dt}, J_{\mathrm{P}-\mathrm{H}}=15.6\right.$ $\mathrm{Hz}, J=7.8 \mathrm{~Hz}, 18 \mathrm{H}), 1.37\left(\mathrm{dm}, J_{\mathrm{P}-\mathrm{H}}=52.2 \mathrm{~Hz}, 12 \mathrm{H}\right), 7.13(\mathrm{t}, J=7.4 \mathrm{~Hz}, 1 \mathrm{H}), 7.27-7.41(\mathrm{~m}, 4 \mathrm{H}), 7.65$ $(\mathrm{d}, J=7.2 \mathrm{~Hz}, 1 \mathrm{H}), 8.49(\mathrm{~d}, J=7.2 \mathrm{~Hz}, 1 \mathrm{H}) ;{ }^{13} \mathrm{C}\left\{{ }^{1} \mathrm{H}\right\} \mathrm{NMR}\left(151 \mathrm{MHz}, \mathrm{CD}_{2} \mathrm{Cl}_{2}\right): \delta 8.0,15.4$ (apparent triplet due to virtual coupling, $\left.{ }^{1+3} J_{\mathrm{P}-\mathrm{C}}=14 \mathrm{~Hz}\right), 121.4\left(\mathrm{t}, J_{\mathrm{P}-\mathrm{C}}=3.0 \mathrm{~Hz}\right), 123.2,124.6,124.8\left(\mathrm{t}, J_{\mathrm{P}-\mathrm{C}}=2.8\right.$ $\mathrm{Hz}), 128.2,132.7,132.8\left(\mathrm{t}, J_{\mathrm{P}-\mathrm{C}}=4.7 \mathrm{~Hz}\right), 133.3\left(\mathrm{t}, J_{\mathrm{P}-\mathrm{C}}=2.3 \mathrm{~Hz}\right), 140.7\left(\mathrm{~d}, J_{\mathrm{P}-\mathrm{C}}=2.3 \mathrm{~Hz}\right), 143.0\left(\mathrm{t}, J_{\mathrm{P}-}\right.$ $\mathrm{C}=26.4 \mathrm{~Hz}), 162.6\left(\mathrm{t}, J_{\mathrm{P}-\mathrm{C}}=30.4 \mathrm{~Hz}\right) ;{ }^{31} \mathrm{P}\left\{{ }^{1} \mathrm{H}\right\} \mathrm{NMR}\left(243 \mathrm{MHz}, \mathrm{CD}_{2} \mathrm{Cl}_{2}\right): \delta$ 18.4. FT-IR $(\mathrm{KBr}): 3044$, 2963, 2926, 2870, 2360, 2093, 1541, 1493, 1452, 1418, 1369, 1258, 1038, 955, 799, 768, 725, $637 \mathrm{~cm}^{-}$

1. Anal. Calcd for $\mathrm{C}_{23} \mathrm{H}_{37} \mathrm{NP}_{2} \mathrm{Ni}$ : C, 61.64; H, 8.32; N, 3.13\%. Found: C, 61.62; H, 8.61; N, 3.05\%. 


\subsection{Reversible Interconversion between 12 and 13}<smiles>CCN(CC)[N+](C#N)(CC)c1cccc2ccccc12</smiles>

13

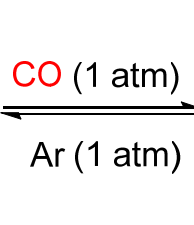<smiles>CCP[N+](C#N)(C(=O)c1cccc2ccccc12)P(CC)CC</smiles>

12

An oven-dried Schlenk tube charged with pure $13(92 \mathrm{mg}, 0.2 \mathrm{mmol})$ in $\mathrm{C}_{7} \mathrm{D}_{8}(0.6 \mathrm{~mL})$ was evacuated and refilled with $\mathrm{CO}$ from a balloon three times. After the reaction mixture was stirred at room temperature for $3 \mathrm{~h}$, the solution was transferred to an NMR tube to measure the ${ }^{31} \mathrm{P}\left\{{ }^{1} \mathrm{H}\right\} \mathrm{NMR}$ spectroscopy. The ${ }^{31} \mathrm{P}\left\{{ }^{1} \mathrm{H}\right\}$ NMR spectrum showed the formation of 12 in $41 \%$ yield, along with complex 13 unreacted in 36\% yield. Then, $\mathrm{CO}$ was replaced with argon again. After $3 \mathrm{~h}, 73 \%$ yield of 13 was observed with no trace of complex 12 and $27 \%$ of the unidentified product. 


\section{Crystallographic Data of Complexes 10 and 13}

\section{Table S15. Crystal Data and Structure Refinement for Complex 10}

Empirical formula

Formula weight

Temperature

Wavelength

Crystal system

Space group

Unit cell dimensions

Volume

Z

Density (calculated)

Absorption coefficient

$\mathrm{F}(000)$

Crystal size

Theta range for data collection

Index ranges

Reflections collected

Independent reflections

Completeness to theta $=27.50^{\circ}$

Max. and min. transmission

Refinement method

Data / restraints / parameters

Goodness-of-fit on $\mathrm{F}^{2}$

Final $\mathrm{R}$ indices [I $>2 \operatorname{sigma}(\mathrm{I})]$

$\mathrm{R}$ indices (all data)

Largest diff. peak and hole
C23 H37 Cl Ni O P2

485.63

153(2) K

$0.71075 \AA$

monoclinic

$P 2{ }_{1} / n$

$\mathrm{a}=12.148(4) \AA$

$\alpha=90^{\circ}$.

$\mathrm{b}=14.698(5) \AA$

$\beta=89.854(5)^{\circ}$.

$\mathrm{c}=13.823(5) \AA$

$\gamma=90^{\circ}$

2468.1(15) $\AA^{3}$

4

$1.307 \mathrm{Mg} / \mathrm{m}^{3}$

$1.035 \mathrm{~mm}^{-1}$

1032

$0.14 \times 0.13 \times 0.03 \mathrm{~mm}^{3}$

2.02 to $27.50^{\circ}$.

$-15<=\mathrm{h}<=11,-17<=\mathrm{k}<=18,-17<=\mathrm{l}<=17$

20488

$5630[\mathrm{R}(\mathrm{int})=0.0317]$

$99.5 \%$

0.9696 and 0.8686

Full-matrix least-squares on $\mathrm{F}^{2}$

$5630 / 30 / 249$

1.109

$\mathrm{R} 1=0.0405, \mathrm{wR} 2=0.0855$

$\mathrm{R} 1=0.0490, \mathrm{wR} 2=0.0952$

0.752 and -0.534 e. $\AA^{-3}$ 
Table S16. Atomic Coordinates ( $x \mathbf{1 0}^{4}$ ) and Equivalent Isotropic Displacement Parameters $\left(\AA^{2} \times 1^{3}\right)$ for Crystalclear. U(eq) is Defined as one Third of the Trace of the Orthogonalized $U^{i j}$ Tensor

\begin{tabular}{|c|c|c|c|c|}
\hline & $\mathrm{x}$ & y & $\mathrm{z}$ & $\mathrm{U}(\mathrm{eq})$ \\
\hline $\mathrm{Ni}(1)$ & $5895(1)$ & $2426(1)$ & $7452(1)$ & $25(1)$ \\
\hline $\mathrm{P}(1)$ & $5797(1)$ & $2535(1)$ & $9047(1)$ & $27(1)$ \\
\hline $\mathrm{P}(2)$ & $6025(1)$ & 2251(1) & $5860(1)$ & $35(1)$ \\
\hline $\mathrm{Cl}(1)$ & $4216(1)$ & $3068(1)$ & $7330(1)$ & $38(1)$ \\
\hline$C(1)$ & 7232(2) & $1804(2)$ & $7567(2)$ & $26(1)$ \\
\hline$C(2)$ & $8285(2)$ & $2348(2)$ & 7496(3) & $25(1)$ \\
\hline$C(3)$ & $8179(2)$ & $3279(2)$ & 7432(3) & $29(1)$ \\
\hline$C(4)$ & 9097(3) & $3862(2)$ & $7345(2)$ & $37(1)$ \\
\hline$C(5)$ & $10125(3)$ & 3499(3) & 7311(3) & $38(1)$ \\
\hline$C(6)$ & 10294(2) & 2551(2) & 7375(3) & $34(1)$ \\
\hline$C(7)$ & 11381(3) & 2181(3) & $7335(3)$ & $43(1)$ \\
\hline $\mathrm{C}(8)$ & $11545(3)$ & $1269(3)$ & 7401(3) & $48(1)$ \\
\hline$C(9)$ & 10644(3) & $676(3)$ & $7518(3)$ & $46(1)$ \\
\hline $\mathrm{C}(10)$ & $9587(3)$ & $1005(2)$ & 7564(3) & $38(1)$ \\
\hline $\mathrm{C}(11)$ & $9373(2)$ & 1949(2) & $7486(2)$ & $28(1)$ \\
\hline$C(12)$ & $4625(3)$ & $1858(3)$ & $9505(3)$ & $39(1)$ \\
\hline $\mathrm{C}(13)$ & 4503(4) & $931(3)$ & $9025(3)$ & $54(1)$ \\
\hline$C(14)$ & $5431(3)$ & $3671(3)$ & 9495(3) & $45(1)$ \\
\hline $\mathrm{C}(15)$ & $6317(4)$ & 4371(3) & 9351(3) & $60(1)$ \\
\hline$C(16)$ & 6976(3) & $2249(2)$ & 9824(2) & $36(1)$ \\
\hline $\mathrm{C}(17)$ & $7140(3)$ & 1251(3) & $10062(3)$ & $45(1)$ \\
\hline$C(18)$ & $6581(5)$ & $3246(3)$ & 5197(4) & $38(2)$ \\
\hline$C(19)$ & $5881(6)$ & 4098(4) & $5352(5)$ & $52(2)$ \\
\hline $\mathrm{C}(20)$ & 4791(4) & 1971(4) & 5191(4) & $36(1)$ \\
\hline $\mathrm{C}(21)$ & $4323(6)$ & 1115(4) & 5632(5) & $47(2)$ \\
\hline $\mathrm{C}(22)$ & 7032(6) & $1403(5)$ & 5422(4) & $34(2)$ \\
\hline$C(23)$ & $7128(5)$ & $1177(5)$ & $4337(4)$ & $33(1)$ \\
\hline $\mathrm{C}(24)$ & $5641(7)$ & $3277(4)$ & $5179(6)$ & $34(2)$ \\
\hline$C(25)$ & $6529(9)$ & $4004(7)$ & $5286(8)$ & $51(3)$ \\
\hline
\end{tabular}




$\begin{array}{llccc}\mathrm{C}(26) & 4758(5) & 1616(5) & 5546(7) & 32(2) \\ \mathrm{C}(27) & 4631(10) & 691(6) & 6074(8) & 51(3) \\ \mathrm{C}(28) & 7173(8) & 1597(10) & 5362(7) & 49(5) \\ \mathrm{C}(29) & 7054(9) & 1546(9) & 4266(7) & 43(3) \\ \mathrm{O}(1) & 7221(2) & 982(1) & 7677(2) & 33(1)\end{array}$

Table S17. Bond Lengths $[\AA ̊]$ and Angles $\left[^{\circ}\right]$ for Crystalclear

\begin{tabular}{ll}
\hline $\mathrm{Ni}(1)-\mathrm{C}(1)$ & $1.870(3)$ \\
$\mathrm{Ni}(1)-\mathrm{P}(1)$ & $2.2133(12)$ \\
$\mathrm{Ni}(1)-\mathrm{P}(2)$ & $2.2207(12)$ \\
$\mathrm{Ni}(1)-\mathrm{Cl}(1)$ & $2.2553(10)$ \\
$\mathrm{P}(1)-\mathrm{C}(14)$ & $1.835(4)$ \\
$\mathrm{P}(1)-\mathrm{C}(16)$ & $1.841(4)$ \\
$\mathrm{P}(1)-\mathrm{C}(12)$ & $1.847(4)$ \\
$\mathrm{P}(2)-\mathrm{C}(20)$ & $1.810(4)$ \\
$\mathrm{P}(2)-\mathrm{C}(28)$ & $1.827(4)$ \\
$\mathrm{P}(2)-\mathrm{C}(24)$ & $1.838(4)$ \\
$\mathrm{P}(2)-\mathrm{C}(22)$ & $1.848(4)$ \\
$\mathrm{P}(2)-\mathrm{C}(26)$ & $1.852(4)$ \\
$\mathrm{P}(2)-\mathrm{C}(18)$ & $1.853(4)$ \\
$\mathrm{C}(1)-\mathrm{O}(1)$ & $1.218(3)$ \\
$\mathrm{C}(1)-\mathrm{C}(2)$ & $1.511(4)$ \\
$\mathrm{C}(2)-\mathrm{C}(3)$ & $1.377(4)$ \\
$\mathrm{C}(2)-\mathrm{C}(11)$ & $1.446(4)$ \\
$\mathrm{C}(3)-\mathrm{C}(4)$ & $1.412(4)$ \\
$\mathrm{C}(4)-\mathrm{C}(5)$ & $1.359(5)$ \\
$\mathrm{C}(5)-\mathrm{C}(6)$ & $1.412(5)$ \\
$\mathrm{C}(6)-\mathrm{C}(7)$ & $1.429(5)$ \\
$\mathrm{C}(6)-\mathrm{C}(11)$ & $1.434(4)$ \\
$\mathrm{C}(7)-\mathrm{C}(8)$ & $1.358(5)$ \\
$\mathrm{C}(8)-\mathrm{C}(9)$ & $1.409(5)$ \\
$\mathrm{C}(9)-\mathrm{C}(10)$ & $1.372(4)$ \\
$\mathrm{C}(10)-\mathrm{C}(11)$ & $1.417(5)$ \\
$\mathrm{C}(12)-\mathrm{C}(13)$ & $1.524(6)$ \\
& \\
& \\
&
\end{tabular}




\begin{tabular}{|c|c|}
\hline $\mathrm{C}(14)-\mathrm{C}(15)$ & $1.502(6)$ \\
\hline$C(16)-C(17)$ & $1.516(5)$ \\
\hline$C(18)-C(19)$ & $1.528(6)$ \\
\hline$C(20)-C(21)$ & $1.508(6)$ \\
\hline$C(22)-C(23)$ & $1.540(6)$ \\
\hline$C(24)-C(25)$ & $1.527(8)$ \\
\hline$C(26)-C(27)$ & $1.550(8)$ \\
\hline C(28)-C(29) & $1.524(8)$ \\
\hline $\mathrm{C}(1)-\mathrm{Ni}(1)-\mathrm{P}(1)$ & $89.73(10)$ \\
\hline $\mathrm{C}(1)-\mathrm{Ni}(1)-\mathrm{P}(2)$ & $88.22(10)$ \\
\hline $\mathrm{P}(1)-\mathrm{Ni}(1)-\mathrm{P}(2)$ & $177.36(4)$ \\
\hline $\mathrm{C}(1)-\mathrm{Ni}(1)-\mathrm{Cl}(1)$ & $175.46(9)$ \\
\hline $\mathrm{P}(1)-\mathrm{Ni}(1)-\mathrm{Cl}(1)$ & $89.84(4)$ \\
\hline $\mathrm{P}(2)-\mathrm{Ni}(1)-\mathrm{Cl}(1)$ & $92.07(4)$ \\
\hline$C(14)-P(1)-C(16)$ & $101.43(18)$ \\
\hline $\mathrm{C}(14)-\mathrm{P}(1)-\mathrm{C}(12)$ & $100.84(18)$ \\
\hline $\mathrm{C}(16)-\mathrm{P}(1)-\mathrm{C}(12)$ & $106.09(17)$ \\
\hline $\mathrm{C}(14)-\mathrm{P}(1)-\mathrm{Ni}(1)$ & $114.53(13)$ \\
\hline $\mathrm{C}(16)-\mathrm{P}(1)-\mathrm{Ni}(1)$ & $121.65(12)$ \\
\hline $\mathrm{C}(12)-\mathrm{P}(1)-\mathrm{Ni}(1)$ & $109.99(14)$ \\
\hline $\mathrm{C}(20)-\mathrm{P}(2)-\mathrm{C}(28)$ & $108.7(5)$ \\
\hline $\mathrm{C}(20)-\mathrm{P}(2)-\mathrm{C}(24)$ & $73.3(3)$ \\
\hline $\mathrm{C}(28)-\mathrm{P}(2)-\mathrm{C}(24)$ & $115.7(5)$ \\
\hline$C(20)-P(2)-C(22)$ & $103.2(3)$ \\
\hline $\mathrm{C}(28)-\mathrm{P}(2)-\mathrm{C}(22)$ & $10.7(6)$ \\
\hline $\mathrm{C}(24)-\mathrm{P}(2)-\mathrm{C}(22)$ & $123.7(4)$ \\
\hline$C(20)-P(2)-C(26)$ & $22.5(3)$ \\
\hline $\mathrm{C}(28)-\mathrm{P}(2)-\mathrm{C}(26)$ & $106.3(6)$ \\
\hline $\mathrm{C}(24)-\mathrm{P}(2)-\mathrm{C}(26)$ & $94.7(4)$ \\
\hline $\mathrm{C}(22)-\mathrm{P}(2)-\mathrm{C}(26)$ & $97.7(4)$ \\
\hline $\mathrm{C}(20)-\mathrm{P}(2)-\mathrm{C}(18)$ & $103.2(3)$ \\
\hline $\mathrm{C}(28)-\mathrm{P}(2)-\mathrm{C}(18)$ & $87.2(5)$ \\
\hline $\mathrm{C}(24)-\mathrm{P}(2)-\mathrm{C}(18)$ & $36.1(3)$ \\
\hline $\mathrm{C}(22)-\mathrm{P}(2)-\mathrm{C}(18)$ & $97.5(3)$ \\
\hline
\end{tabular}




$\begin{array}{ll}\mathrm{C}(26)-\mathrm{P}(2)-\mathrm{C}(18) & 125.7(3) \\ \mathrm{C}(20)-\mathrm{P}(2)-\mathrm{Ni}(1) & 118.4(2) \\ \mathrm{C}(28)-\mathrm{P}(2)-\mathrm{Ni}(1) & 119.1(3) \\ \mathrm{C}(24)-\mathrm{P}(2)-\mathrm{Ni}(1) & 113.3(3) \\ \mathrm{C}(22)-\mathrm{P}(2)-\mathrm{Ni}(1) & 116.6(2) \\ \mathrm{C}(26)-\mathrm{P}(2)-\mathrm{Ni}(1) & 103.5(3) \\ \mathrm{C}(18)-\mathrm{P}(2)-\mathrm{Ni}(1) & 115.10(19) \\ \mathrm{O}(1)-\mathrm{C}(1)-\mathrm{C}(2) & 122.8(3) \\ \mathrm{O}(1)-\mathrm{C}(1)-\mathrm{Ni}(1) & 119.0(2) \\ \mathrm{C}(2)-\mathrm{C}(1)-\mathrm{Ni}(1) & 118.1(2) \\ \mathrm{C}(3)-\mathrm{C}(2)-\mathrm{C}(11) & 119.2(2) \\ \mathrm{C}(3)-\mathrm{C}(2)-\mathrm{C}(1) & 116.8(2) \\ \mathrm{C}(11)-\mathrm{C}(2)-\mathrm{C}(1) & 124.0(2) \\ \mathrm{C}(2)-\mathrm{C}(3)-\mathrm{C}(4) & 122.3(3) \\ \mathrm{C}(5)-\mathrm{C}(4)-\mathrm{C}(3) & 119.3(3) \\ \mathrm{C}(4)-\mathrm{C}(5)-\mathrm{C}(6) & 121.3(3) \\ \mathrm{C}(5)-\mathrm{C}(6)-\mathrm{C}(7) & 120.5(3) \\ \mathrm{C}(5)-\mathrm{C}(6)-\mathrm{C}(11) & 120.1(3) \\ \mathrm{C}(7)-\mathrm{C}(6)-\mathrm{C}(11) & 119.4(3) \\ \mathrm{C}(8)-\mathrm{C}(7)-\mathrm{C}(6) & 120.6(3) \\ \mathrm{C}(7)-\mathrm{C}(8)-\mathrm{C}(9) & 120.3(3) \\ \mathrm{C}(10)-\mathrm{C}(9)-\mathrm{C}(8) & 120.9(3) \\ \mathrm{C}(9)-\mathrm{C}(10)-\mathrm{C}(11) & 120.9(3) \\ \mathrm{C}(10)-\mathrm{C}(11)-\mathrm{C}(6) & 117.9(3) \\ \mathrm{C}(10)-\mathrm{C}(11)-\mathrm{C}(2) & 124.4(3) \\ \mathrm{C}(6)-\mathrm{C}(11)-\mathrm{C}(2) & 117.7(3) \\ \mathrm{C}(13)-\mathrm{C}(12)-\mathrm{P}(1) & 114.1(3) \\ \mathrm{C}(15)-\mathrm{C}(14)-\mathrm{P}(1) & 113.9(3) \\ \mathrm{C}(17)-\mathrm{C}(16)-\mathrm{P}(1) & 116.7(3) \\ \mathrm{C}(19)-\mathrm{C}(18)-\mathrm{P}(2) & 112.0(4) \\ \mathrm{C}(21)-\mathrm{C}(20)-\mathrm{P}(2) & 107.1(4) \\ \mathrm{C}(23)-\mathrm{C}(22)-\mathrm{P}(2) & 120.8(4) \\ \mathrm{C}(25)-\mathrm{C}(24)-\mathrm{P}(2) & 110.2(6) \\ \mathrm{C}(27)-\mathrm{C}(26)-\mathrm{P}(2) & 114.4(6) \\ \mathrm{C}(29)-\mathrm{C}(28)-\mathrm{P}(2) & 109.1(7)\end{array}$


Symmetry transformations used to generate equivalent atoms:

Table S18. Anisotropic Displacement Parameters $\left(\AA^{2} \times 10^{3}\right)$ for Crystalclear. The Anisotropic Displacement Factor Exponent Takes the Form: $-2 \square{ }^{2}\left[h^{\mathbf{2}} a^{* 2} U^{11}+\ldots+\right.$ 2 h k a* b* $\mathbf{U}^{12}$ ]

\begin{tabular}{|c|c|c|c|c|c|c|}
\hline & $\mathrm{U}^{11}$ & $\mathrm{U}^{22}$ & $\mathrm{U}^{33}$ & $\mathrm{U}^{23}$ & $\mathrm{U}^{13}$ & $\mathrm{U}^{12}$ \\
\hline $\mathrm{Ni}(1)$ & $21(1)$ & $27(1)$ & $27(1)$ & $1(1)$ & $-1(1)$ & $-2(1)$ \\
\hline $\mathrm{P}(1)$ & $27(1)$ & $29(1)$ & $25(1)$ & $1(1)$ & $4(1)$ & $0(1)$ \\
\hline $\mathrm{P}(2)$ & $49(1)$ & $29(1)$ & $27(1)$ & 1(1) & $-10(1)$ & $-3(1)$ \\
\hline $\mathrm{Cl}(1)$ & $25(1)$ & $45(1)$ & $44(1)$ & $2(1)$ & $-1(1)$ & $5(1)$ \\
\hline $\mathrm{C}(1)$ & $32(1)$ & $24(2)$ & $22(1)$ & $-1(1)$ & $-1(1)$ & $-4(1)$ \\
\hline$C(2)$ & $21(1)$ & $30(2)$ & $23(1)$ & $-2(1)$ & $1(1)$ & $-2(1)$ \\
\hline$C(3)$ & $27(1)$ & $30(2)$ & $32(2)$ & $2(1)$ & $-2(1)$ & $-4(1)$ \\
\hline$C(4)$ & $41(2)$ & $31(2)$ & $39(2)$ & $3(1)$ & $-7(2)$ & $-9(2)$ \\
\hline$C(5)$ & $33(2)$ & $46(2)$ & $35(2)$ & $6(2)$ & $-2(2)$ & $-18(2)$ \\
\hline$C(6)$ & $25(1)$ & $53(2)$ & $23(2)$ & $-1(2)$ & $0(1)$ & $-4(1)$ \\
\hline$C(7)$ & $28(2)$ & $68(3)$ & $33(2)$ & $3(2)$ & $3(2)$ & $-2(2)$ \\
\hline $\mathrm{C}(8)$ & $29(2)$ & $77(3)$ & $37(2)$ & $-4(2)$ & $1(2)$ & $12(2)$ \\
\hline$C(9)$ & $40(2)$ & $49(2)$ & $48(2)$ & $-2(2)$ & $-1(2)$ & $16(2)$ \\
\hline$C(10)$ & $33(2)$ & $42(2)$ & $40(2)$ & $-1(2)$ & $1(2)$ & $5(1)$ \\
\hline $\mathrm{C}(11)$ & $27(1)$ & $34(2)$ & $24(1)$ & $2(1)$ & 1(1) & $1(1)$ \\
\hline$C(12)$ & $30(2)$ & $46(2)$ & $43(2)$ & $13(2)$ & $11(2)$ & $1(2)$ \\
\hline$C(13)$ & $43(2)$ & $46(2)$ & $72(3)$ & $15(2)$ & $2(2)$ & $-17(2)$ \\
\hline$C(14)$ & $55(2)$ & $36(2)$ & $42(2)$ & $-5(2)$ & $6(2)$ & $8(2)$ \\
\hline$C(15)$ & $85(4)$ & $31(2)$ & $63(3)$ & $-7(2)$ & $13(3)$ & $-1(2)$ \\
\hline$C(16)$ & $33(2)$ & $46(2)$ & $28(2)$ & $3(2)$ & $-2(1)$ & $-3(2)$ \\
\hline$C(17)$ & $45(2)$ & $50(2)$ & $41(2)$ & $2(2)$ & $-4(2)$ & $7(2)$ \\
\hline $\mathrm{O}(1)$ & $34(1)$ & $25(1)$ & $41(1)$ & $2(1)$ & $2(1)$ & $-2(1)$ \\
\hline
\end{tabular}


Table S19. Hydrogen Coordinates ( $x \mathbf{1 0}^{4}$ ) and Isotropic Displacement Parameters $\left(\AA^{2} \times 10^{3}\right)$ for Crystalclear

\begin{tabular}{|c|c|c|c|c|}
\hline & $\mathrm{x}$ & $\mathrm{y}$ & $\mathrm{z}$ & $\mathrm{U}(\mathrm{eq})$ \\
\hline $\mathrm{H}(1)$ & 7462 & 3538 & 7446 & 35 \\
\hline $\mathrm{H}(2)$ & 8997 & 4503 & 7311 & 44 \\
\hline $\mathrm{H}(3)$ & 10742 & 3891 & 7242 & 45 \\
\hline $\mathrm{H}(4)$ & 11994 & 2576 & 7262 & 51 \\
\hline $\mathrm{H}(5)$ & 12271 & 1030 & 7369 & 58 \\
\hline $\mathrm{H}(6)$ & 10768 & 39 & 7565 & 55 \\
\hline $\mathrm{H}(7)$ & 8992 & 593 & 7649 & 46 \\
\hline $\mathrm{H}(8)$ & 4715 & 1769 & 10210 & 47 \\
\hline $\mathrm{H}(9)$ & 3938 & 2208 & 9404 & 47 \\
\hline $\mathrm{H}(10)$ & 3870 & 612 & 9305 & 80 \\
\hline $\mathrm{H}(11)$ & 5172 & 572 & 9133 & 80 \\
\hline $\mathrm{H}(12)$ & 4390 & 1011 & 8329 & 80 \\
\hline $\mathrm{H}(13)$ & 4756 & 3877 & 9161 & 53 \\
\hline $\mathrm{H}(14)$ & 5261 & 3628 & 10195 & 53 \\
\hline $\mathrm{H}(15)$ & 6068 & 4957 & 9610 & 89 \\
\hline $\mathrm{H}(16)$ & 6475 & 4434 & 8659 & 89 \\
\hline $\mathrm{H}(17)$ & 6985 & 4180 & 9690 & 89 \\
\hline $\mathrm{H}(18)$ & 7653 & 2475 & 9506 & 43 \\
\hline $\mathrm{H}(19)$ & 6893 & 2586 & 10440 & 43 \\
\hline $\mathrm{H}(20)$ & 7783 & 1183 & 10482 & 68 \\
\hline $\mathrm{H}(21)$ & 7258 & 909 & 9462 & 68 \\
\hline $\mathrm{H}(22)$ & 6485 & 1018 & 10394 & 68 \\
\hline $\mathrm{H}(23)$ & 7342 & 3367 & 5417 & 45 \\
\hline $\mathrm{H}(24)$ & 6611 & 3103 & 4497 & 45 \\
\hline $\mathrm{H}(25)$ & 6203 & 4607 & 4991 & 78 \\
\hline $\mathrm{H}(26)$ & 5860 & 4247 & 6042 & 78 \\
\hline $\mathrm{H}(27)$ & 5131 & 3985 & 5121 & 78 \\
\hline $\mathrm{H}(28)$ & 4250 & 2474 & 5238 & 43 \\
\hline $\mathrm{H}(29)$ & 4969 & 1871 & 4500 & 43 \\
\hline
\end{tabular}




\begin{tabular}{lrrrr}
$\mathrm{H}(30)$ & 3653 & 940 & 5285 & 70 \\
$\mathrm{H}(31)$ & 4146 & 1225 & 6314 & 70 \\
$\mathrm{H}(32)$ & 4867 & 624 & 5584 & 70 \\
$\mathrm{H}(33)$ & 7768 & 1607 & 5639 & 41 \\
$\mathrm{H}(34)$ & 6874 & 5764 & 41 \\
$\mathrm{H}(35)$ & 7708 & 723 & 4240 & 49 \\
$\mathrm{H}(36)$ & 7312 & 1731 & 3976 & 49 \\
$\mathrm{H}(37)$ & 9426 & 4106 & 49 \\
$\mathrm{H}(38)$ & 5548 & 3122 & 4487 & 41 \\
$\mathrm{H}(39)$ & 3514 & 5425 & 41 \\
$\mathrm{H}(40)$ & 4548 & 4922 & 76 \\
$\mathrm{H}(41)$ & 3930 & 5771 & 5033 & 76 \\
$\mathrm{H}(42)$ & 6315 & 4161 & 5971 & 76 \\
$\mathrm{H}(43)$ & 7229 & 2000 & 5702 & 39 \\
$\mathrm{H}(44)$ & 6614 & 1506 & 4840 & 39 \\
$\mathrm{H}(45)$ & 4112 & 401 & 5875 & 76 \\
$\mathrm{H}(46)$ & 4753 & 792 & 6775 & 76 \\
$\mathrm{H}(47)$ & 3942 & 5906 & 76 \\
$\mathrm{H}(48)$ & 4619 & 1197 & 393 & 59 \\
$\mathrm{H}(49)$ & 5253 & 5530 & 59 \\
$\mathrm{H}(50)$ & 7878 & 5639 & 65 \\
$\mathrm{H}(51)$ & 7173 & 3996 & 65 \\
$\mathrm{H}(52)$ & 7669 & 4104 & 65 \\
\hline & 7059 & &
\end{tabular}




\section{Table S20. Crystal Data and Structure Refinement for Complex 13}

Empirical formula

Formula weight

Temperature

Wavelength

Crystal system

Space group

Unit cell dimensions

Volume

Z

Density (calculated)

Absorption coefficient

$\mathrm{F}(000)$

Crystal size

Theta range for data collection

Index ranges

Reflections collected

Independent reflections

Completeness to theta $=26.00^{\circ}$

Max. and min. transmission

Refinement method

Data / restraints / parameters

Goodness-of-fit on $\mathrm{F}^{2}$

Final $\mathrm{R}$ indices [I $>2 \operatorname{sigma}(\mathrm{I})]$

$\mathrm{R}$ indices (all data)

Absolute structure parameter

Largest diff. peak and hole

\section{C23 H37 N Ni P2}

448.19

$153(2) \mathrm{K}$

$0.71075 \AA$

orthorhombic

$P_{n a 2}$

$\mathrm{a}=18.336(4) \AA$

$\square=90^{\circ}$.

$\mathrm{b}=10.341(2) \AA$

$\square=90^{\circ}$.

$\mathrm{c}=12.785(3) \AA$

$\square=90^{\circ}$.

2424.2(9) $\AA^{3}$

4

$1.228 \mathrm{Mg} / \mathrm{m}^{3}$

$0.940 \mathrm{~mm}^{-1}$

960

$0.22 \times 0.21 \times 0.18 \mathrm{~mm}^{3}$

2.26 to $26.00^{\circ}$.

$-22<=\mathrm{h}<=22,-12<=\mathrm{k}<=12,-15<=\mathrm{l}<=15$

17007

$4640[\mathrm{R}(\mathrm{int})=0.0257]$

$99.1 \%$

0.8490 and 0.8199

Full-matrix least-squares on $\mathrm{F}^{2}$

4640 / 133 / 235

1.077

$\mathrm{R} 1=0.0917, \mathrm{wR} 2=0.2381$

$\mathrm{R} 1=0.1064, \mathrm{wR} 2=0.2528$

$0.47(5)$

2.298 and -1.513 e. $\AA^{-3}$ 
Table S21. Atomic Coordinates ( $\left.x 1^{4}\right)$ and Equivalent Isotropic Displacement Parameters $\left(\AA^{2} \times 1^{3}\right)$ for Crystalclear. $U(e q)$ is Defined as one Third of the Trace of the Orthogonalized $U^{i j}$ Tensor

\begin{tabular}{|c|c|c|c|c|}
\hline & $\mathrm{x}$ & $\mathrm{y}$ & $\mathrm{z}$ & $\mathrm{U}(\mathrm{eq})$ \\
\hline $\mathrm{Ni}(1)$ & $9082(1)$ & $7850(1)$ & $5652(2)$ & $54(1)$ \\
\hline $\mathrm{C}(1)$ & 9719(4) & $9304(6)$ & $5653(14)$ & $56(2)$ \\
\hline $\mathrm{N}(1)$ & $10075(3)$ & $10211(6)$ & $5654(15)$ & $72(2)$ \\
\hline$C(2)$ & $8275(3)$ & $6586(7)$ & $5649(12)$ & $54(2)$ \\
\hline$C(3)$ & 7578(4) & 6997(7) & $5636(13)$ & $62(2)$ \\
\hline $\mathrm{C}(4)$ & 6970(3) & $6129(6)$ & $5662(12)$ & $52(1)$ \\
\hline$C(5)$ & 7063(4) & $4834(7)$ & $5651(13)$ & $59(2)$ \\
\hline$C(6)$ & 7831(4) & $4345(6)$ & $5645(12)$ & $56(2)$ \\
\hline$C(7)$ & $7946(5)$ & $3020(7)$ & $5642(16)$ & $73(2)$ \\
\hline $\mathrm{C}(8)$ & $8641(5)$ & 2591(9) & $5696(15)$ & $74(2)$ \\
\hline $\mathrm{C}(9)$ & $9256(4)$ & $3446(6)$ & $5659(12)$ & $53(2)$ \\
\hline$C(10)$ & 9131(4) & $4721(7)$ & $5638(13)$ & $54(2)$ \\
\hline$C(11)$ & $8386(3)$ & $5277(5)$ & $5647(10)$ & $42(1)$ \\
\hline $\mathrm{P}(1)$ & $9098(2)$ & 7848(3) & $3956(2)$ & $59(1)$ \\
\hline $\mathrm{P}(2)$ & $9120(2)$ & 7834(3) & $7354(2)$ & $64(1)$ \\
\hline$C(12)$ & $8860(40)$ & $6330(30)$ & $3274(19)$ & $80(30)$ \\
\hline$C(13)$ & $8978(13)$ & $6540(20)$ & $2093(15)$ & $20(5)$ \\
\hline$C(14)$ & $8391(16)$ & $8960(30)$ & $3370(20)$ & $52(8)$ \\
\hline$C(15)$ & $8290(20)$ & $10230(30)$ & $3960(30)$ & $67(10)$ \\
\hline$C(16)$ & $9770(20)$ & $8320(60)$ & $2990(40)$ & $120(30)$ \\
\hline$C(17)$ & $10356(19)$ & $7390(40)$ & $3390(30)$ & $44(8)$ \\
\hline$C(18)$ & $8719(14)$ & $6431(15)$ & $3282(11)$ & $53(5)$ \\
\hline C(19) & $8691(12)$ & $6391(18)$ & $2087(11)$ & $72(5)$ \\
\hline$C(20)$ & $8813(7)$ & $9432(12)$ & $3480(12)$ & $57(4)$ \\
\hline$C(21)$ & $7995(8)$ & $9675(18)$ & $3723(14)$ & $72(5)$ \\
\hline$C(22)$ & $10071(6)$ & 7934(14) & $3517(12)$ & $50(4)$ \\
\hline$C(23)$ & 10541(10) & $6798(18)$ & $3870(15)$ & $75(5)$ \\
\hline$C(24)$ & $8620(8)$ & $6646(13)$ & $8046(8)$ & $40(3)$ \\
\hline$C(25)$ & $8778(9)$ & $6638(18)$ & $9215(10)$ & $49(4)$ \\
\hline
\end{tabular}




\begin{tabular}{lrlll}
$\mathrm{C}(26)$ & $8717(8)$ & $9323(14)$ & $7890(14)$ & $59(5)$ \\
$\mathrm{C}(27)$ & $7898(9)$ & $9230(20)$ & $7789(19)$ & $99(7)$ \\
$\mathrm{C}(28)$ & $10054(8)$ & $8000(20)$ & $7830(20)$ & $71(6)$ \\
$\mathrm{C}(29)$ & $10526(11)$ & $6820(20)$ & $7622(18)$ & $69(5)$ \\
$\mathrm{C}(30)$ & $8808(16)$ & $6299(19)$ & $8028(14)$ & $34(6)$ \\
$\mathrm{C}(31)$ & $8825(12)$ & $6040(20)$ & $9223(13)$ & $35(5)$ \\
$\mathrm{C}(32)$ & $8298(15)$ & $8530(30)$ & $8000(30)$ & $89(11)$ \\
$\mathrm{C}(33)$ & $8168(17)$ & $9900(30)$ & $7620(30)$ & $64(8)$ \\
$\mathrm{C}(34)$ & $9873(12)$ & $8250(30)$ & $8140(20)$ & $45(6)$ \\
$\mathrm{C}(35)$ & $10493(17)$ & $7370(40)$ & $7760(30)$ & $67(10)$ \\
\hline
\end{tabular}

Table S22. Bond Lengths $[\AA ̊]$ and Angles $\left[^{\circ}\right]$ for Crystalclear

\begin{tabular}{ll}
\hline $\mathrm{Ni}(1)-\mathrm{C}(1)$ & $1.905(7)$ \\
$\mathrm{Ni}(1)-\mathrm{C}(2)$ & $1.975(6)$ \\
$\mathrm{Ni}(1)-\mathrm{P}(1)$ & $2.169(4)$ \\
$\mathrm{Ni}(1)-\mathrm{P}(2)$ & $2.177(3)$ \\
$\mathrm{C}(1)-\mathrm{N}(1)$ & $1.142(8)$ \\
$\mathrm{C}(2)-\mathrm{C}(3)$ & $1.346(10)$ \\
$\mathrm{C}(2)-\mathrm{C}(11)$ & $1.369(9)$ \\
$\mathrm{C}(3)-\mathrm{C}(4)$ & $1.432(9)$ \\
$\mathrm{C}(4)-\mathrm{C}(5)$ & $1.350(9)$ \\
$\mathrm{C}(5)-\mathrm{C}(6)$ & $1.496(9)$ \\
$\mathrm{C}(6)-\mathrm{C}(7)$ & $1.386(10)$ \\
$\mathrm{C}(6)-\mathrm{C}(11)$ & $1.401(8)$ \\
$\mathrm{C}(7)-\mathrm{C}(8)$ & $1.350(12)$ \\
$\mathrm{C}(8)-\mathrm{C}(9)$ & $1.435(11)$ \\
$\mathrm{C}(9)-\mathrm{C}(10)$ & $1.339(9)$ \\
$\mathrm{C}(10)-\mathrm{C}(11)$ & $1.484(8)$ \\
$\mathrm{P}(1)-\mathrm{C}(16)$ & $1.804(16)$ \\
$\mathrm{P}(1)-\mathrm{C}(20)$ & $1.824(10)$ \\
$\mathrm{P}(1)-\mathrm{C}(18)$ & $1.837(11)$ \\
$\mathrm{P}(1)-\mathrm{C}(12)$ & $1.847(16)$ \\
$\mathrm{P}(1)-\mathrm{C}(22)$ & $1.873(10)$ \\
$\mathrm{P}(1)-\mathrm{C}(14)$ & $1.888(14)$ \\
\end{tabular}




$\begin{array}{lc}\mathrm{P}(2)-\mathrm{C}(34) & 1.759(13) \\ \mathrm{P}(2)-\mathrm{C}(24) & 1.769(10) \\ \mathrm{P}(2)-\mathrm{C}(28) & 1.827(13) \\ \mathrm{P}(2)-\mathrm{C}(26) & 1.840(11) \\ \mathrm{P}(2)-\mathrm{C}(32) & 1.862(15) \\ \mathrm{P}(2)-\mathrm{C}(30) & 1.894(13) \\ \mathrm{C}(12)-\mathrm{C}(13) & 1.540(16) \\ \mathrm{C}(14)-\mathrm{C}(15) & 1.529(16) \\ \mathrm{C}(16)-\mathrm{C}(17) & 1.533(17) \\ \mathrm{C}(18)-\mathrm{C}(19) & 1.530(12) \\ \mathrm{C}(20)-\mathrm{C}(21) & 1.552(13) \\ \mathrm{C}(22)-\mathrm{C}(23) & 1.525(13) \\ \mathrm{C}(24)-\mathrm{C}(25) & 1.522(12) \\ \mathrm{C}(26)-\mathrm{C}(27) & 1.510(14) \\ \mathrm{C}(28)-\mathrm{C}(29) & 1.515(14) \\ \mathrm{C}(30)-\mathrm{C}(31) & 1.553(14) \\ \mathrm{C}(32)-\mathrm{C}(33) & 1.520(16) \\ \mathrm{C}(34)-\mathrm{C}(35) & 1.531(16) \\ \mathrm{C}(1)-\mathrm{Ni}(1)-\mathrm{C}(2) & \\ \mathrm{C}(1)-\mathrm{Ni}(1)-\mathrm{P}(1) & 169.3(3) \\ \mathrm{C}(2)-\mathrm{Ni}(1)-\mathrm{P}(1) & 89.6(5) \\ \mathrm{C}(1)-\mathrm{Ni}(1)-\mathrm{P}(2) & 90.4(4) \\ \mathrm{C}(2)-\mathrm{Ni}(1)-\mathrm{P}(2) & 89.2(5) \\ \mathrm{P}(1)-\mathrm{Ni}(1)-\mathrm{P}(2) & 91.2(4) \\ \mathrm{N}(1)-\mathrm{C}(1)-\mathrm{Ni}(1) & 177.35(9) \\ \mathrm{C}(3)-\mathrm{C}(2)-\mathrm{C}(11) & 177.0(6) \\ \mathrm{C}(3)-\mathrm{C}(2)-\mathrm{Ni}(1) & 116.9(6) \\ \mathrm{C}(11)-\mathrm{C}(2)-\mathrm{Ni}(1) & 120.2(5) \\ \mathrm{C}(2)-\mathrm{C}(3)-\mathrm{C}(4) & 122.9(5) \\ \mathrm{C}(5)-\mathrm{C}(4)-\mathrm{C}(3) & 122.7(7) \\ \mathrm{C}(4)-\mathrm{C}(5)-\mathrm{C}(6) & 121.6(6) \\ \mathrm{C}(7)-\mathrm{C}(6)-\mathrm{C}(11) & \\ \mathrm{C}(11)-\mathrm{C}(6)-\mathrm{C}(5)-\mathrm{C}(5) & \\ & 117.0(6) \\ & \end{array}$




\begin{tabular}{|c|c|}
\hline $\mathrm{C}(8)-\mathrm{C}(7)-\mathrm{C}(6)$ & $117.9(8)$ \\
\hline $\mathrm{C}(7)-\mathrm{C}(8)-\mathrm{C}(9)$ & $122.6(8)$ \\
\hline$C(10)-C(9)-C(8)$ & $118.2(6)$ \\
\hline$C(9)-C(10)-C(11)$ & $122.6(6)$ \\
\hline$C(2)-C(11)-C(6)$ & $124.9(6)$ \\
\hline$C(2)-C(11)-C(10)$ & $121.3(5)$ \\
\hline $\mathrm{C}(6)-\mathrm{C}(11)-\mathrm{C}(10)$ & $113.8(5)$ \\
\hline $\mathrm{C}(16)-\mathrm{P}(1)-\mathrm{C}(20)$ & $74.0(15)$ \\
\hline $\mathrm{C}(16)-\mathrm{P}(1)-\mathrm{C}(18)$ & $99(3)$ \\
\hline $\mathrm{C}(20)-\mathrm{P}(1)-\mathrm{C}(18)$ & $116.8(9)$ \\
\hline$C(16)-P(1)-C(12)$ & $94(3)$ \\
\hline $\mathrm{C}(20)-\mathrm{P}(1)-\mathrm{C}(12)$ & $123(2)$ \\
\hline $\mathrm{C}(18)-\mathrm{P}(1)-\mathrm{C}(12)$ & $9(3)$ \\
\hline$C(16)-P(1)-C(22)$ & $30.2(14)$ \\
\hline$C(20)-P(1)-C(22)$ & $97.5(6)$ \\
\hline $\mathrm{C}(18)-\mathrm{P}(1)-\mathrm{C}(22)$ & 104.9(9) \\
\hline $\mathrm{C}(12)-\mathrm{P}(1)-\mathrm{C}(22)$ & $97(2)$ \\
\hline $\mathrm{C}(16)-\mathrm{P}(1)-\mathrm{C}(14)$ & $91.8(17)$ \\
\hline $\mathrm{C}(20)-\mathrm{P}(1)-\mathrm{C}(14)$ & $28.8(11)$ \\
\hline $\mathrm{C}(18)-\mathrm{P}(1)-\mathrm{C}(14)$ & $92.3(12)$ \\
\hline $\mathrm{C}(12)-\mathrm{P}(1)-\mathrm{C}(14)$ & $100(3)$ \\
\hline $\mathrm{C}(22)-\mathrm{P}(1)-\mathrm{C}(14)$ & $120.5(12)$ \\
\hline $\mathrm{C}(16)-\mathrm{P}(1)-\mathrm{Ni}(1)$ & $134(2)$ \\
\hline $\mathrm{C}(20)-\mathrm{P}(1)-\mathrm{Ni}(1)$ & 109.2(5) \\
\hline $\mathrm{C}(18)-\mathrm{P}(1)-\mathrm{Ni}(1)$ & $117.7(5)$ \\
\hline $\mathrm{C}(12)-\mathrm{P}(1)-\mathrm{Ni}(1)$ & $118.0(9)$ \\
\hline $\mathrm{C}(22)-\mathrm{P}(1)-\mathrm{Ni}(1)$ & 108.3(5) \\
\hline $\mathrm{C}(14)-\mathrm{P}(1)-\mathrm{Ni}(1)$ & $112.7(10)$ \\
\hline $\mathrm{C}(34)-\mathrm{P}(2)-\mathrm{C}(24)$ & $106.9(10)$ \\
\hline $\mathrm{C}(34)-\mathrm{P}(2)-\mathrm{C}(28)$ & $18.3(10)$ \\
\hline $\mathrm{C}(24)-\mathrm{P}(2)-\mathrm{C}(28)$ & 112.4(9) \\
\hline $\mathrm{C}(34)-\mathrm{P}(2)-\mathrm{C}(26)$ & $84.3(11)$ \\
\hline $\mathrm{C}(24)-\mathrm{P}(2)-\mathrm{C}(26)$ & $100.8(8)$ \\
\hline $\mathrm{C}(28)-\mathrm{P}(2)-\mathrm{C}(26)$ & 100.1(9) \\
\hline $\mathrm{C}(34)-\mathrm{P}(2)-\mathrm{C}(32)$ & $106.8(16)$ \\
\hline
\end{tabular}




$\begin{array}{lc}\mathrm{C}(24)-\mathrm{P}(2)-\mathrm{C}(32) & 68.1(11) \\ \mathrm{C}(28)-\mathrm{P}(2)-\mathrm{C}(32) & 125.0(15) \\ \mathrm{C}(26)-\mathrm{P}(2)-\mathrm{C}(32) & 35.7(12) \\ \mathrm{C}(34)-\mathrm{P}(2)-\mathrm{C}(30) & 100.4(12) \\ \mathrm{C}(24)-\mathrm{P}(2)-\mathrm{C}(30) & 15.2(9) \\ \mathrm{C}(28)-\mathrm{P}(2)-\mathrm{C}(30) & 101.9(12) \\ \mathrm{C}(26)-\mathrm{P}(2)-\mathrm{C}(30) & 114.3(11) \\ \mathrm{C}(32)-\mathrm{P}(2)-\mathrm{C}(30) & 82.9(13) \\ \mathrm{C}(34)-\mathrm{P}(2)-\mathrm{Ni}(1) & 126.4(10) \\ \mathrm{C}(24)-\mathrm{P}(2)-\mathrm{Ni}(1) & 119.2(4) \\ \mathrm{C}(28)-\mathrm{P}(2)-\mathrm{Ni}(1) & 111.4(9) \\ \mathrm{C}(26)-\mathrm{P}(2)-\mathrm{Ni}(1) & 110.7(6) \\ \mathrm{C}(32)-\mathrm{P}(2)-\mathrm{Ni}(1) & 114.5(12) \\ \mathrm{C}(30)-\mathrm{P}(2)-\mathrm{Ni}(1) & 116.8(6) \\ \mathrm{C}(13)-\mathrm{C}(12)-\mathrm{P}(1) & 108.3(16) \\ \mathrm{C}(15)-\mathrm{C}(14)-\mathrm{P}(1) & 115(2) \\ \mathrm{C}(17)-\mathrm{C}(16)-\mathrm{P}(1) & 95(2) \\ \mathrm{C}(19)-\mathrm{C}(18)-\mathrm{P}(1) & 120.2(11) \\ \mathrm{C}(21)-\mathrm{C}(20)-\mathrm{P}(1) & 110.9(10) \\ \mathrm{C}(23)-\mathrm{C}(22)-\mathrm{P}(1) & 114.3(11) \\ \mathrm{C}(25)-\mathrm{C}(24)-\mathrm{P}(2) & 113.3(9) \\ \mathrm{C}(27)-\mathrm{C}(26)-\mathrm{P}(2) & 108.3(13) \\ \mathrm{C}(29)-\mathrm{C}(28)-\mathrm{P}(2) & 113.7(13) \\ \mathrm{C}(31)-\mathrm{C}(30)-\mathrm{P}(2) & 126.0(15) \\ \mathrm{C}(33)-\mathrm{C}(32)-\mathrm{P}(2) & 110.2(19) \\ \mathrm{C}(35)-\mathrm{C}(34)-\mathrm{P}(2) & 105.1(19) \\ & \end{array}$

Symmetry transformations used to generate equivalent atoms:

Table S23. Anisotropic Displacement Parameters $\left(\AA^{2} \times 1^{3}\right)$ for Crystalclear. The Anisotropic Displacement Factor Exponent Takes the Form: $-2 \pi^{2}\left[h^{2} a * 2 U^{11}+\ldots+\right.$ 2 h k a* b* U12 ]

$\begin{array}{llllll}\mathrm{U}^{11} & \mathrm{U}^{22} & \mathrm{U}^{33} & \mathrm{U}^{23} & \mathrm{U}^{13} & \mathrm{U}^{12}\end{array}$




\begin{tabular}{lllllll}
$\mathrm{Ni}(1)$ & $77(1)$ & $42(1)$ & $41(1)$ & $0(1)$ & $2(1)$ & $-13(1)$ \\
$\mathrm{C}(1)$ & $58(4)$ & $43(3)$ & $65(4)$ & $-1(8)$ & $13(7)$ & $5(3)$ \\
$\mathrm{N}(1)$ & $62(3)$ & $49(3)$ & $104(5)$ & $-6(8)$ & $-1(9)$ & $-4(3)$ \\
$\mathrm{C}(2)$ & $55(3)$ & $70(4)$ & $38(3)$ & $-7(7)$ & $7(6)$ & $-5(3)$ \\
$\mathrm{C}(3)$ & $74(5)$ & $71(4)$ & $41(3)$ & $0(7)$ & $18(7)$ & $-2(3)$ \\
$\mathrm{C}(4)$ & $43(3)$ & $72(4)$ & $41(3)$ & $6(7)$ & $6(6)$ & $0(3)$ \\
$\mathrm{C}(5)$ & $59(4)$ & $71(4)$ & $46(3)$ & $3(8)$ & $-2(7)$ & $-4(3)$ \\
$\mathrm{C}(6)$ & $70(4)$ & $63(4)$ & $33(3)$ & $-2(7)$ & $3(6)$ & $-14(3)$ \\
$\mathrm{C}(7)$ & $69(5)$ & $64(4)$ & $85(5)$ & $16(9)$ & $32(8)$ & $-6(3)$ \\
$\mathrm{C}(8)$ & $81(5)$ & $82(5)$ & $59(5)$ & $-1(9)$ & $2(9)$ & $7(4)$ \\
$\mathrm{C}(9)$ & $68(4)$ & $58(4)$ & $34(3)$ & $-15(6)$ & $-4(6)$ & $14(3)$ \\
$\mathrm{C}(10)$ & $63(4)$ & $62(4)$ & $36(3)$ & $10(7)$ & $9(6)$ & $9(3)$ \\
$\mathrm{C}(11)$ & $50(3)$ & $53(3)$ & $25(2)$ & $0(5)$ & $4(5)$ & $-12(2)$ \\
$\mathrm{P}(1)$ & $74(2)$ & $52(3)$ & $50(2)$ & $15(1)$ & $18(2)$ & $4(1)$ \\
$\mathrm{P}(2)$ & $101(3)$ & $58(4)$ & $32(2)$ & $-10(1)$ & $-9(2)$ & $2(2)$ \\
\hline
\end{tabular}

Table S24. Hydrogen Coordinates ( $x 1^{4}$ ) and Isotropic Displacement Parameters $\left(\AA^{2} \times 10^{3}\right)$ for Crystalclear

\begin{tabular}{lrrrl}
\hline & $x$ & $y$ & $U(e q)$ \\
& & & & \\
$H(1)$ & 7486 & 7900 & 5608 & 74 \\
$\mathrm{H}(2)$ & 6490 & 6472 & 5689 & 62 \\
$\mathrm{H}(3)$ & 6658 & 4259 & 5648 & 70 \\
$\mathrm{H}(4)$ & 7549 & 2433 & 5604 & 87 \\
$\mathrm{H}(5)$ & 8725 & 1689 & 5760 & 89 \\
$\mathrm{H}(6)$ & 9740 & 3116 & 5650 & 64 \\
$\mathrm{H}(7)$ & 9537 & 5293 & 5617 & 64 \\
$\mathrm{H}(8)$ & 9176 & 5617 & 3526 & 99 \\
$\mathrm{H}(9)$ & 8347 & 6100 & 3414 & 99 \\
$\mathrm{H}(10)$ & 8659 & 5950 & 1701 & 30 \\
$\mathrm{H}(11)$ & 9488 & 6359 & 1914 & 30 \\
$\mathrm{H}(12)$ & 8859 & 7433 & 1911 & 30 \\
$\mathrm{H}(13)$ & 8531 & 9153 & 2640 & 63 \\
& & & &
\end{tabular}




\begin{tabular}{|c|c|c|c|c|}
\hline $\mathrm{H}(14)$ & 7917 & 8499 & 3352 & 63 \\
\hline $\mathrm{H}(15)$ & 7883 & 10717 & 3646 & 100 \\
\hline $\mathrm{H}(16)$ & 8736 & 10749 & 3908 & 100 \\
\hline $\mathrm{H}(17)$ & 8181 & 10056 & 4694 & 100 \\
\hline $\mathrm{H}(18)$ & 9610 & 8118 & 2268 & 149 \\
\hline $\mathrm{H}(19)$ & 9908 & 9238 & 3049 & 149 \\
\hline $\mathrm{H}(20)$ & 10805 & 7519 & 2990 & 66 \\
\hline $\mathrm{H}(21)$ & 10189 & 6499 & 3317 & 66 \\
\hline $\mathrm{H}(22)$ & 10452 & 7574 & 4135 & 66 \\
\hline $\mathrm{H}(23)$ & 9002 & 5668 & 3515 & 63 \\
\hline $\mathrm{H}(24)$ & 8214 & 6312 & 3539 & 63 \\
\hline $\mathrm{H}(25)$ & 8879 & 5558 & 1840 & 107 \\
\hline $\mathrm{H}(26)$ & 8990 & 7093 & 1801 & 107 \\
\hline $\mathrm{H}(27)$ & 8185 & 6496 & 1853 & 107 \\
\hline $\mathrm{H}(28)$ & 9112 & 10112 & 3817 & 69 \\
\hline $\mathrm{H}(29)$ & 8893 & 9482 & 2715 & 69 \\
\hline $\mathrm{H}(30)$ & 7850 & 10520 & 3444 & 108 \\
\hline $\mathrm{H}(31)$ & 7919 & 9662 & 4482 & 108 \\
\hline $\mathrm{H}(32)$ & 7699 & 8997 & 3396 & 108 \\
\hline $\mathrm{H}(33)$ & 10082 & 7977 & 2743 & 60 \\
\hline H(34) & 10289 & 8744 & 3786 & 60 \\
\hline $\mathrm{H}(35)$ & 11040 & 6916 & 3615 & 112 \\
\hline $\mathrm{H}(36)$ & 10340 & 5993 & 3588 & 112 \\
\hline $\mathrm{H}(37)$ & 10544 & 6757 & 4636 & 112 \\
\hline $\mathrm{H}(38)$ & 8092 & 6801 & 7937 & 48 \\
\hline H(39) & 8737 & 5784 & 7753 & 48 \\
\hline $\mathrm{H}(40)$ & 8522 & 5911 & 9542 & 73 \\
\hline $\mathrm{H}(41)$ & 8609 & 7451 & 9526 & 73 \\
\hline $\mathrm{H}(42)$ & 9304 & 6546 & 9328 & 73 \\
\hline $\mathrm{H}(43)$ & 8855 & 9423 & 8634 & 70 \\
\hline $\mathrm{H}(44)$ & 8899 & 10084 & 7500 & 70 \\
\hline $\mathrm{H}(45)$ & 7674 & 10015 & 8074 & 148 \\
\hline $\mathrm{H}(46)$ & 7723 & 8476 & 8178 & 148 \\
\hline $\mathrm{H}(47)$ & 7766 & 9141 & 7050 & 148 \\
\hline $\mathrm{H}(48)$ & 10281 & 8761 & 7502 & 85 \\
\hline
\end{tabular}




\begin{tabular}{lrrrr}
$\mathrm{H}(49)$ & 10040 & 8152 & 8598 & 85 \\
$\mathrm{H}(50)$ & 11008 & 6951 & 7937 & 104 \\
$\mathrm{H}(51)$ & 10579 & 6705 & 6865 & 104 \\
$\mathrm{H}(52)$ & 10297 & 6054 & 7926 & 104 \\
$\mathrm{H}(53)$ & 8296 & 6168 & 7810 & 41 \\
$\mathrm{H}(54)$ & 9092 & 5589 & 7704 & 41 \\
$\mathrm{H}(55)$ & 8627 & 5174 & 9365 & 52 \\
$\mathrm{H}(56)$ & 8530 & 6688 & 9585 & 52 \\
$\mathrm{H}(57)$ & 9329 & 6082 & 9475 & 52 \\
$\mathrm{H}(58)$ & 7867 & 7986 & 7838 & 107 \\
$\mathrm{H}(59)$ & 8368 & 8528 & 8768 & 107 \\
$\mathrm{H}(60)$ & 7735 & 10256 & 7966 & 95 \\
$\mathrm{H}(61)$ & 8093 & 9896 & 6862 & 95 \\
$\mathrm{H}(62)$ & 8593 & 10436 & 7790 & 95 \\
$\mathrm{H}(63)$ & 10003 & 9168 & 8046 & 54 \\
$\mathrm{H}(64)$ & 9765 & 8089 & 8887 & 54 \\
$\mathrm{H}(65)$ & 10943 & 7596 & 8135 & 101 \\
$\mathrm{H}(66)$ & 10564 & 7488 & 7010 & 101 \\
$\mathrm{H}(67)$ & 10368 & 6466 & 7907 & 101 \\
\hline
\end{tabular}

Table S25. Torsion Angles $\left[^{\circ}\right]$ for Crystalclear

\begin{tabular}{lc}
\hline $\mathrm{C}(2)-\mathrm{Ni}(1)-\mathrm{C}(1)-\mathrm{N}(1)$ & $1(36)$ \\
$\mathrm{P}(1)-\mathrm{Ni}(1)-\mathrm{C}(1)-\mathrm{N}(1)$ & $91(34)$ \\
$\mathrm{P}(2)-\mathrm{Ni}(1)-\mathrm{C}(1)-\mathrm{N}(1)$ & $-91(34)$ \\
$\mathrm{C}(1)-\mathrm{Ni}(1)-\mathrm{C}(2)-\mathrm{C}(3)$ & $0(5)$ \\
$\mathrm{P}(1)-\mathrm{Ni}(1)-\mathrm{C}(2)-\mathrm{C}(3)$ & $-89.8(13)$ \\
$\mathrm{P}(2)-\mathrm{Ni}(1)-\mathrm{C}(2)-\mathrm{C}(3)$ & $92.3(13)$ \\
$\mathrm{C}(1)-\mathrm{Ni}(1)-\mathrm{C}(2)-\mathrm{C}(11)$ & $180(3)$ \\
$\mathrm{P}(1)-\mathrm{Ni}(1)-\mathrm{C}(2)-\mathrm{C}(11)$ & $89.4(13)$ \\
$\mathrm{P}(2)-\mathrm{Ni}(1)-\mathrm{C}(2)-\mathrm{C}(11)$ & $-88.5(12)$ \\
$\mathrm{C}(11)-\mathrm{C}(2)-\mathrm{C}(3)-\mathrm{C}(4)$ & $3(2)$ \\
$\mathrm{Ni}(1)-\mathrm{C}(2)-\mathrm{C}(3)-\mathrm{C}(4)$ & $-178.2(11)$ \\
$\mathrm{C}(2)-\mathrm{C}(3)-\mathrm{C}(4)-\mathrm{C}(5)$ & $-3(3)$ \\
$\mathrm{C}(3)-\mathrm{C}(4)-\mathrm{C}(5)-\mathrm{C}(6)$ & $2(2)$
\end{tabular}




$\begin{array}{lc}\mathrm{C}(4)-\mathrm{C}(5)-\mathrm{C}(6)-\mathrm{C}(7) & 179.5(17) \\ \mathrm{C}(4)-\mathrm{C}(5)-\mathrm{C}(6)-\mathrm{C}(11) & 0(2) \\ \mathrm{C}(11)-\mathrm{C}(6)-\mathrm{C}(7)-\mathrm{C}(8) & 3(3) \\ \mathrm{C}(5)-\mathrm{C}(6)-\mathrm{C}(7)-\mathrm{C}(8) & -176.3(16) \\ \mathrm{C}(6)-\mathrm{C}(7)-\mathrm{C}(8)-\mathrm{C}(9) & -6(3) \\ \mathrm{C}(7)-\mathrm{C}(8)-\mathrm{C}(9)-\mathrm{C}(10) & 4(3) \\ \mathrm{C}(8)-\mathrm{C}(9)-\mathrm{C}(10)-\mathrm{C}(11) & 0(3) \\ \mathrm{C}(3)-\mathrm{C}(2)-\mathrm{C}(11)-\mathrm{C}(6) & -1(2) \\ \mathrm{Ni}(1)-\mathrm{C}(2)-\mathrm{C}(11)-\mathrm{C}(6) & 180.0(10) \\ \mathrm{C}(3)-\mathrm{C}(2)-\mathrm{C}(11)-\mathrm{C}(10) & 178.7(13) \\ \mathrm{Ni}(1)-\mathrm{C}(2)-\mathrm{C}(11)-\mathrm{C}(10) & -1(2) \\ \mathrm{C}(7)-\mathrm{C}(6)-\mathrm{C}(11)-\mathrm{C}(2) & 179.9(16) \\ \mathrm{C}(5)-\mathrm{C}(6)-\mathrm{C}(11)-\mathrm{C}(2) & 0(2) \\ \mathrm{C}(7)-\mathrm{C}(6)-\mathrm{C}(11)-\mathrm{C}(10) & 0(2) \\ \mathrm{C}(5)-\mathrm{C}(6)-\mathrm{C}(11)-\mathrm{C}(10) & -179.9(12) \\ \mathrm{C}(9)-\mathrm{C}(10)-\mathrm{C}(11)-\mathrm{C}(2) & 178.5(15) \\ \mathrm{C}(9)-\mathrm{C}(10)-\mathrm{C}(11)-\mathrm{C}(6) & -2(2) \\ \mathrm{C}(1)-\mathrm{Ni}(1)-\mathrm{P}(1)-\mathrm{C}(16) & 30(2) \\ \mathrm{C}(2)-\mathrm{Ni}(1)-\mathrm{P}(1)-\mathrm{C}(16) & -161(2) \\ \mathrm{P}(2)-\mathrm{Ni}(1)-\mathrm{P}(1)-\mathrm{C}(16) & -33(3) \\ \mathrm{C}(1)-\mathrm{Ni}(1)-\mathrm{P}(1)-\mathrm{C}(20) & -55.8(5) \\ \mathrm{C}(2)-\mathrm{Ni}(1)-\mathrm{P}(1)-\mathrm{C}(20) & 113.4(5) \\ \mathrm{P}(2)-\mathrm{Ni}(1)-\mathrm{P}(1)-\mathrm{C}(20) & -149.3(19) \\ \mathrm{C}(1)-\mathrm{Ni}(1)-\mathrm{P}(1)-\mathrm{C}(18) & \\ \mathrm{C}(2)-\mathrm{Ni}(1)-\mathrm{P}(1)-\mathrm{C}(18) & 167.9(10) \\ \mathrm{P}(2)-\mathrm{Ni}(1)-\mathrm{P}(1)-\mathrm{C}(18) & -22.8(10) \\ \mathrm{C}(1)-\mathrm{Ni}(1)-\mathrm{P}(1)-\mathrm{C}(12) & 105.2(18) \\ \mathrm{C}(2)-\mathrm{Ni}(1)-\mathrm{P}(1)-\mathrm{C}(12) & 158(3) \\ \mathrm{P}(2)-\mathrm{Ni}(1)-\mathrm{P}(1)-\mathrm{C}(12) & -33(3) \\ \mathrm{C}(1)-\mathrm{Ni}(1)-\mathrm{P}(1)-\mathrm{C}(22) & 95(3) \\ \mathrm{C}(2)-\mathrm{Ni}(1)-\mathrm{P}(1)-\mathrm{C}(22) & -141.5(5) \\ \mathrm{P}(2)-\mathrm{Ni}(1)-\mathrm{P}(1)-\mathrm{C}(22) & \\ \mathrm{C}(1)-\mathrm{Ni}(1)-\mathrm{P}(1)-\mathrm{C}(14) & \\ \mathrm{C}(2)-\mathrm{Ni}(1)-\mathrm{P}(1)-\mathrm{C}(14) & \\ \mathrm{P}(2)-\mathrm{Ni}(1)-\mathrm{P}(1)-\mathrm{C}(14) & \\ & \\ & \end{array}$




$\begin{array}{lc}\mathrm{C}(1)-\mathrm{Ni}(1)-\mathrm{P}(2)-\mathrm{C}(34) & -34.3(12) \\ \mathrm{C}(2)-\mathrm{Ni}(1)-\mathrm{P}(2)-\mathrm{C}(34) & 156.4(12) \\ \mathrm{P}(1)-\mathrm{Ni}(1)-\mathrm{P}(2)-\mathrm{C}(34) & 28(2) \\ \mathrm{C}(1)-\mathrm{Ni}(1)-\mathrm{P}(2)-\mathrm{C}(24) & -179.9(7) \\ \mathrm{C}(2)-\mathrm{Ni}(1)-\mathrm{P}(2)-\mathrm{C}(24) & 10.8(7) \\ \mathrm{P}(1)-\mathrm{Ni}(1)-\mathrm{P}(2)-\mathrm{C}(24) & -117.1(17) \\ \mathrm{C}(1)-\mathrm{Ni}(1)-\mathrm{P}(2)-\mathrm{C}(28) & -46.4(7) \\ \mathrm{C}(2)-\mathrm{Ni}(1)-\mathrm{P}(2)-\mathrm{C}(28) & 144.4(7) \\ \mathrm{P}(1)-\mathrm{Ni}(1)-\mathrm{P}(2)-\mathrm{C}(28) & 16.4(18) \\ \mathrm{C}(1)-\mathrm{Ni}(1)-\mathrm{P}(2)-\mathrm{C}(26) & 64.0(6) \\ \mathrm{C}(2)-\mathrm{Ni}(1)-\mathrm{P}(2)-\mathrm{C}(26) & -105.3(6) \\ \mathrm{P}(1)-\mathrm{Ni}(1)-\mathrm{P}(2)-\mathrm{C}(26) & 126.8(16) \\ \mathrm{C}(1)-\mathrm{Ni}(1)-\mathrm{P}(2)-\mathrm{C}(32) & 102.6(12) \\ \mathrm{C}(2)-\mathrm{Ni}(1)-\mathrm{P}(2)-\mathrm{C}(32) & -66.7(12) \\ \mathrm{P}(1)-\mathrm{Ni}(1)-\mathrm{P}(2)-\mathrm{C}(32) & 165.3(19) \\ \mathrm{C}(1)-\mathrm{Ni}(1)-\mathrm{P}(2)-\mathrm{C}(30) & -162.9(11) \\ \mathrm{C}(2)-\mathrm{Ni}(1)-\mathrm{P}(2)-\mathrm{C}(30) & 27.8(11) \\ \mathrm{P}(1)-\mathrm{Ni}(1)-\mathrm{P}(2)-\mathrm{C}(30) & -100.2(19) \\ \mathrm{C}(16)-\mathrm{P}(1)-\mathrm{C}(12)-\mathrm{C}(13) & -30(4) \\ \mathrm{C}(20)-\mathrm{P}(1)-\mathrm{C}(12)-\mathrm{C}(13) & 44(5) \\ \mathrm{C}(18)-\mathrm{P}(1)-\mathrm{C}(12)-\mathrm{C}(13) & -178(3) \\ \mathrm{C}(22)-\mathrm{P}(1)-\mathrm{C}(12)-\mathrm{C}(13) & \\ \mathrm{C}(14)-\mathrm{P}(1)-\mathrm{C}(12)-\mathrm{C}(13) & -65(9) \\ \mathrm{Ni}(1)-\mathrm{P}(1)-\mathrm{C}(12)-\mathrm{C}(13) & -60(4) \\ \mathrm{C}(16)-\mathrm{P}(1)-\mathrm{C}(14)-\mathrm{C}(15) & 63(4) \\ \mathrm{C}(20)-\mathrm{P}(1)-\mathrm{C}(14)-\mathrm{C}(15) & -175(3) \\ \mathrm{C}(18)-\mathrm{P}(1)-\mathrm{C}(14)-\mathrm{C}(15) & -101(4) \\ \mathrm{C}(12)-\mathrm{P}(1)-\mathrm{C}(14)-\mathrm{C}(15) & -50(2) \\ \mathrm{C}(22)-\mathrm{P}(1)-\mathrm{C}(14)-\mathrm{C}(15) & 159(4) \\ \mathrm{Ni}(1)-\mathrm{P}(1)-\mathrm{C}(14)-\mathrm{C}(15) & 160(3) \\ \mathrm{C}(20)-\mathrm{P}(1)-\mathrm{C}(16)-\mathrm{C}(17) & 165(3) \\ \mathrm{C}(18)-\mathrm{P}(1)-\mathrm{C}(16)-\mathrm{C}(17) & -91(3) \\ \mathrm{C}(12)-\mathrm{P}(1)-\mathrm{C}(16)-\mathrm{C}(17) & \\ \mathrm{C}(22)-\mathrm{P}(1)-\mathrm{C}(16)-\mathrm{C}(17) & \\ \mathrm{C}(14)-\mathrm{P}(1)-\mathrm{C}(16)-\mathrm{C}(17) & \\ & \end{array}$




\begin{tabular}{|c|c|}
\hline Ni(1)-P(1)-C(16)-C(17) & $58(5)$ \\
\hline $\mathrm{C}(16)-\mathrm{P}(1)-\mathrm{C}(18)-\mathrm{C}(19)$ & $-31(2)$ \\
\hline $\mathrm{C}(20)-\mathrm{P}(1)-\mathrm{C}(18)-\mathrm{C}(19)$ & $45(2)$ \\
\hline $\mathrm{C}(12)-\mathrm{P}(1)-\mathrm{C}(18)-\mathrm{C}(19)$ & $-88(7)$ \\
\hline $\mathrm{C}(22)-\mathrm{P}(1)-\mathrm{C}(18)-\mathrm{C}(19)$ & $-62(2)$ \\
\hline $\mathrm{C}(14)-\mathrm{P}(1)-\mathrm{C}(18)-\mathrm{C}(19)$ & $61(2)$ \\
\hline $\mathrm{Ni}(1)-\mathrm{P}(1)-\mathrm{C}(18)-\mathrm{C}(19)$ & $177.9(14)$ \\
\hline $\mathrm{C}(16)-\mathrm{P}(1)-\mathrm{C}(20)-\mathrm{C}(21)$ & $160(3)$ \\
\hline $\mathrm{C}(18)-\mathrm{P}(1)-\mathrm{C}(20)-\mathrm{C}(21)$ & $68.7(14)$ \\
\hline $\mathrm{C}(12)-\mathrm{P}(1)-\mathrm{C}(20)-\mathrm{C}(21)$ & $76(3)$ \\
\hline $\mathrm{C}(22)-\mathrm{P}(1)-\mathrm{C}(20)-\mathrm{C}(21)$ & $179.7(11)$ \\
\hline $\mathrm{C}(14)-\mathrm{P}(1)-\mathrm{C}(20)-\mathrm{C}(21)$ & $34(2)$ \\
\hline $\mathrm{Ni}(1)-\mathrm{P}(1)-\mathrm{C}(20)-\mathrm{C}(21)$ & $-68.0(12)$ \\
\hline $\mathrm{C}(16)-\mathrm{P}(1)-\mathrm{C}(22)-\mathrm{C}(23)$ & $-147(5)$ \\
\hline $\mathrm{C}(20)-\mathrm{P}(1)-\mathrm{C}(22)-\mathrm{C}(23)$ & $174.3(13)$ \\
\hline $\mathrm{C}(18)-\mathrm{P}(1)-\mathrm{C}(22)-\mathrm{C}(23)$ & $-65.3(15)$ \\
\hline $\mathrm{C}(12)-\mathrm{P}(1)-\mathrm{C}(22)-\mathrm{C}(23)$ & $-61(2)$ \\
\hline $\mathrm{C}(14)-\mathrm{P}(1)-\mathrm{C}(22)-\mathrm{C}(23)$ & $-167.1(14)$ \\
\hline Ni(1)-P(1)-C(22)-C(23) & $61.2(13)$ \\
\hline $\mathrm{C}(34)-\mathrm{P}(2)-\mathrm{C}(24)-\mathrm{C}(25)$ & $20.6(15)$ \\
\hline $\mathrm{C}(28)-\mathrm{P}(2)-\mathrm{C}(24)-\mathrm{C}(25)$ & $39.1(14)$ \\
\hline $\mathrm{C}(26)-\mathrm{P}(2)-\mathrm{C}(24)-\mathrm{C}(25)$ & $-66.6(12)$ \\
\hline $\mathrm{C}(32)-\mathrm{P}(2)-\mathrm{C}(24)-\mathrm{C}(25)$ & $-81.1(17)$ \\
\hline $\mathrm{C}(30)-\mathrm{P}(2)-\mathrm{C}(24)-\mathrm{C}(25)$ & $87(3)$ \\
\hline $\mathrm{Ni}(1)-\mathrm{P}(2)-\mathrm{C}(24)-\mathrm{C}(25)$ & $172.2(9)$ \\
\hline $\mathrm{C}(34)-\mathrm{P}(2)-\mathrm{C}(26)-\mathrm{C}(27)$ & $-158.0(16)$ \\
\hline $\mathrm{C}(24)-\mathrm{P}(2)-\mathrm{C}(26)-\mathrm{C}(27)$ & $-51.9(15)$ \\
\hline $\mathrm{C}(28)-\mathrm{P}(2)-\mathrm{C}(26)-\mathrm{C}(27)$ & $-167.2(14)$ \\
\hline $\mathrm{C}(32)-\mathrm{P}(2)-\mathrm{C}(26)-\mathrm{C}(27)$ & $-28(2)$ \\
\hline $\mathrm{C}(30)-\mathrm{P}(2)-\mathrm{C}(26)-\mathrm{C}(27)$ & $-59.2(17)$ \\
\hline $\mathrm{Ni}(1)-\mathrm{P}(2)-\mathrm{C}(26)-\mathrm{C}(27)$ & $75.2(14)$ \\
\hline $\mathrm{C}(34)-\mathrm{P}(2)-\mathrm{C}(28)-\mathrm{C}(29)$ & $143(5)$ \\
\hline $\mathrm{C}(24)-\mathrm{P}(2)-\mathrm{C}(28)-\mathrm{C}(29)$ & $68(2)$ \\
\hline $\mathrm{C}(26)-\mathrm{P}(2)-\mathrm{C}(28)-\mathrm{C}(29)$ & $173.7(17)$ \\
\hline $\mathrm{C}(32)-\mathrm{P}(2)-\mathrm{C}(28)-\mathrm{C}(29)$ & $145.7(18)$ \\
\hline
\end{tabular}




$\begin{array}{lc}\mathrm{C}(30)-\mathrm{P}(2)-\mathrm{C}(28)-\mathrm{C}(29) & 56(2) \\ \mathrm{Ni}(1)-\mathrm{P}(2)-\mathrm{C}(28)-\mathrm{C}(29) & -69.3(18) \\ \mathrm{C}(34)-\mathrm{P}(2)-\mathrm{C}(30)-\mathrm{C}(31) & 36(3) \\ \mathrm{C}(24)-\mathrm{P}(2)-\mathrm{C}(30)-\mathrm{C}(31) & -80(3) \\ \mathrm{C}(28)-\mathrm{P}(2)-\mathrm{C}(30)-\mathrm{C}(31) & 55(3) \\ \mathrm{C}(26)-\mathrm{P}(2)-\mathrm{C}(30)-\mathrm{C}(31) & -52(3) \\ \mathrm{C}(32)-\mathrm{P}(2)-\mathrm{C}(30)-\mathrm{C}(31) & -69(3) \\ \mathrm{Ni}(1)-\mathrm{P}(2)-\mathrm{C}(30)-\mathrm{C}(31) & 176.6(19) \\ \mathrm{C}(34)-\mathrm{P}(2)-\mathrm{C}(32)-\mathrm{C}(33) & 89(3) \\ \mathrm{C}(24)-\mathrm{P}(2)-\mathrm{C}(32)-\mathrm{C}(33) & -170(3) \\ \mathrm{C}(28)-\mathrm{P}(2)-\mathrm{C}(32)-\mathrm{C}(33) & 88(3) \\ \mathrm{C}(26)-\mathrm{P}(2)-\mathrm{C}(32)-\mathrm{C}(33) & 35.4(18) \\ \mathrm{C}(30)-\mathrm{P}(2)-\mathrm{C}(32)-\mathrm{C}(33) & -173(3) \\ \mathrm{Ni}(1)-\mathrm{P}(2)-\mathrm{C}(32)-\mathrm{C}(33) & -56(3) \\ \mathrm{C}(24)-\mathrm{P}(2)-\mathrm{C}(34)-\mathrm{C}(35) & 93(2) \\ \mathrm{C}(28)-\mathrm{P}(2)-\mathrm{C}(34)-\mathrm{C}(35) & -17(4) \\ \mathrm{C}(26)-\mathrm{P}(2)-\mathrm{C}(34)-\mathrm{C}(35) & -167(2) \\ \mathrm{C}(32)-\mathrm{P}(2)-\mathrm{C}(34)-\mathrm{C}(35) & 165(2) \\ \mathrm{C}(30)-\mathrm{P}(2)-\mathrm{C}(34)-\mathrm{C}(35) & 79(2) \\ \mathrm{Ni}(1)-\mathrm{P}(2)-\mathrm{C}(34)-\mathrm{C}(35) & -56(2)\end{array}$

Symmetry transformations used to generate equivalent atoms: 


\section{Copies of NMR Spectra}

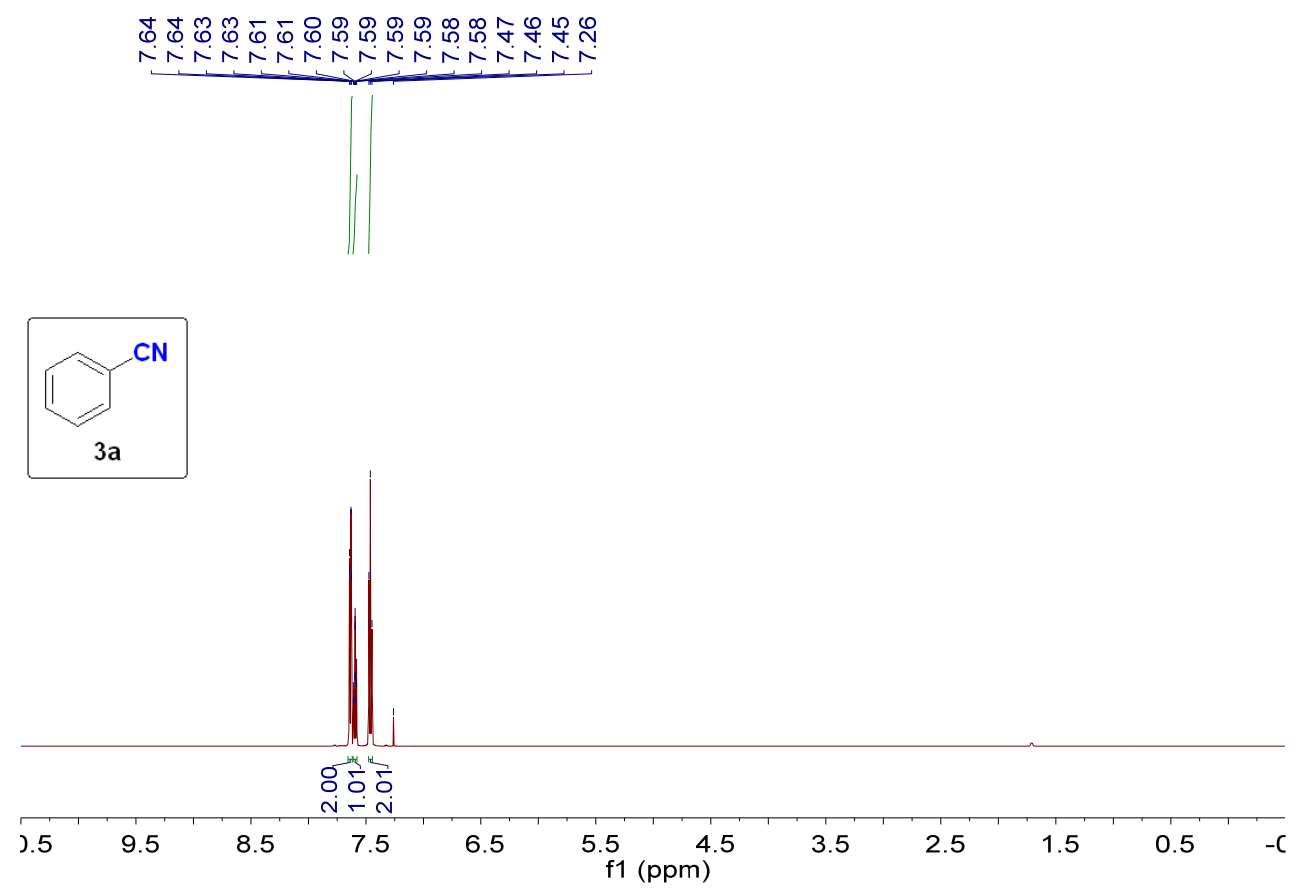

${ }^{1} \mathrm{H}$ NMR (600 MHz) spectrum of $\mathbf{3 a}\left(\mathrm{CDCl}_{3}, \mathrm{rt}\right)$.

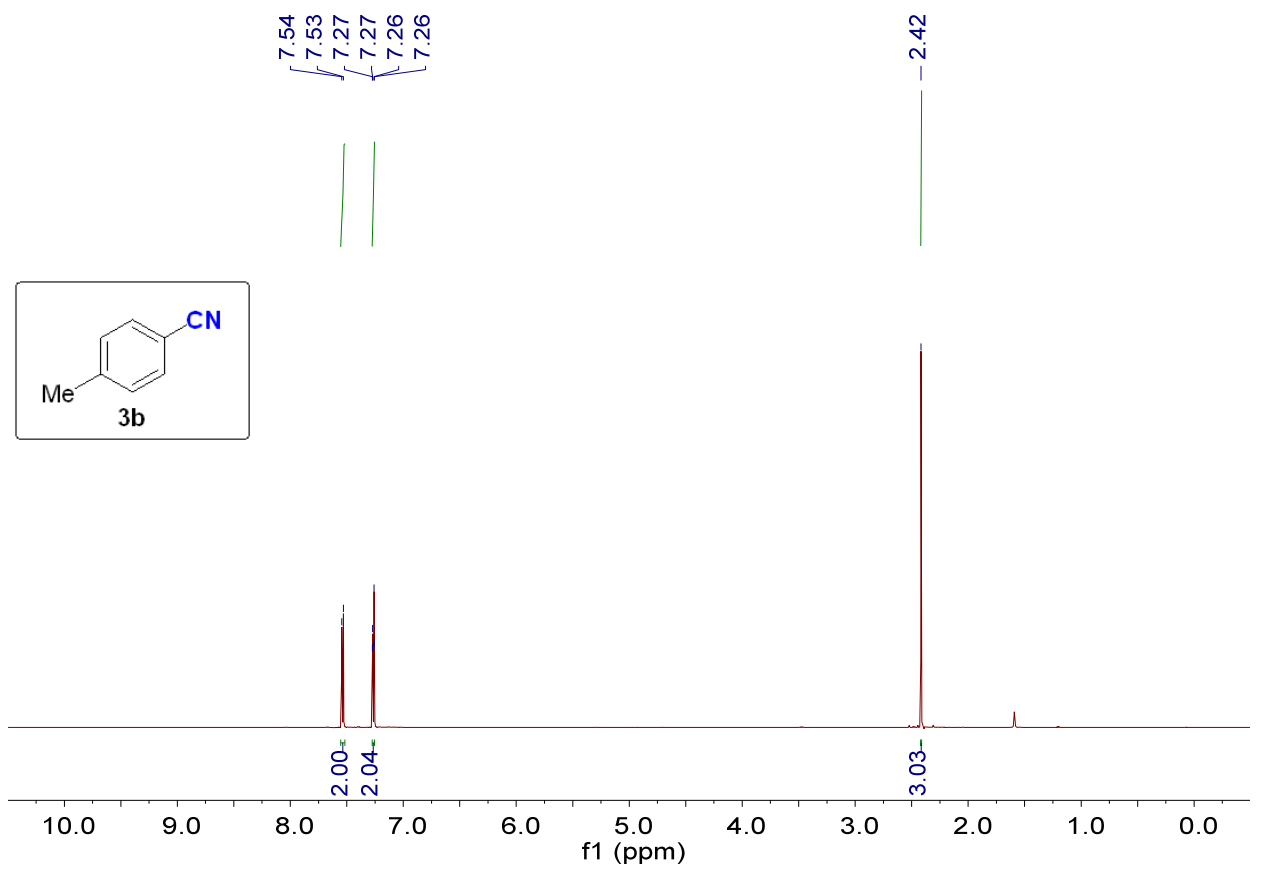

${ }^{1} \mathrm{H} \mathrm{NMR}(600 \mathrm{MHz})$ spectrum of $\mathbf{3 b}\left(\mathrm{CDCl}_{3}, \mathrm{rt}\right)$. 

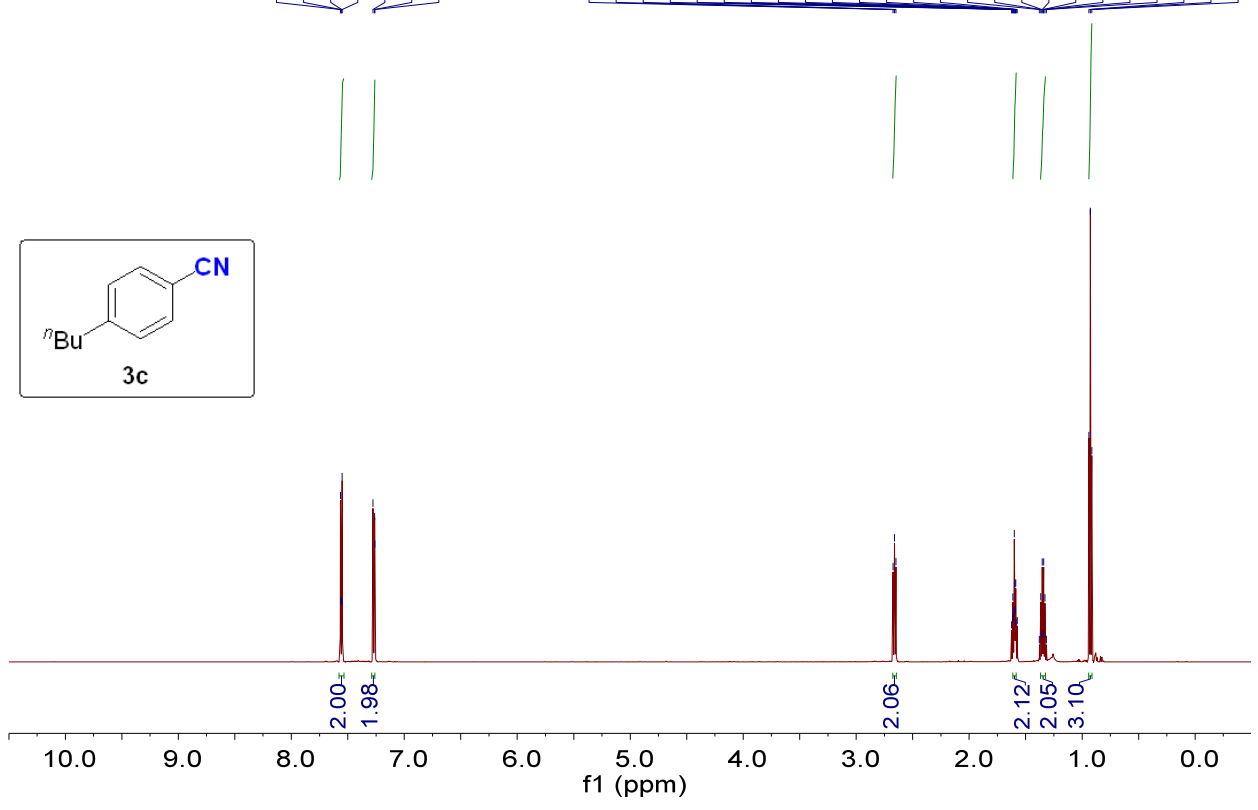

${ }^{1} \mathrm{H}$ NMR $(600 \mathrm{MHz})$ spectrum of $\mathbf{3 c}\left(\mathrm{CDCl}_{3}, \mathrm{rt}\right)$.

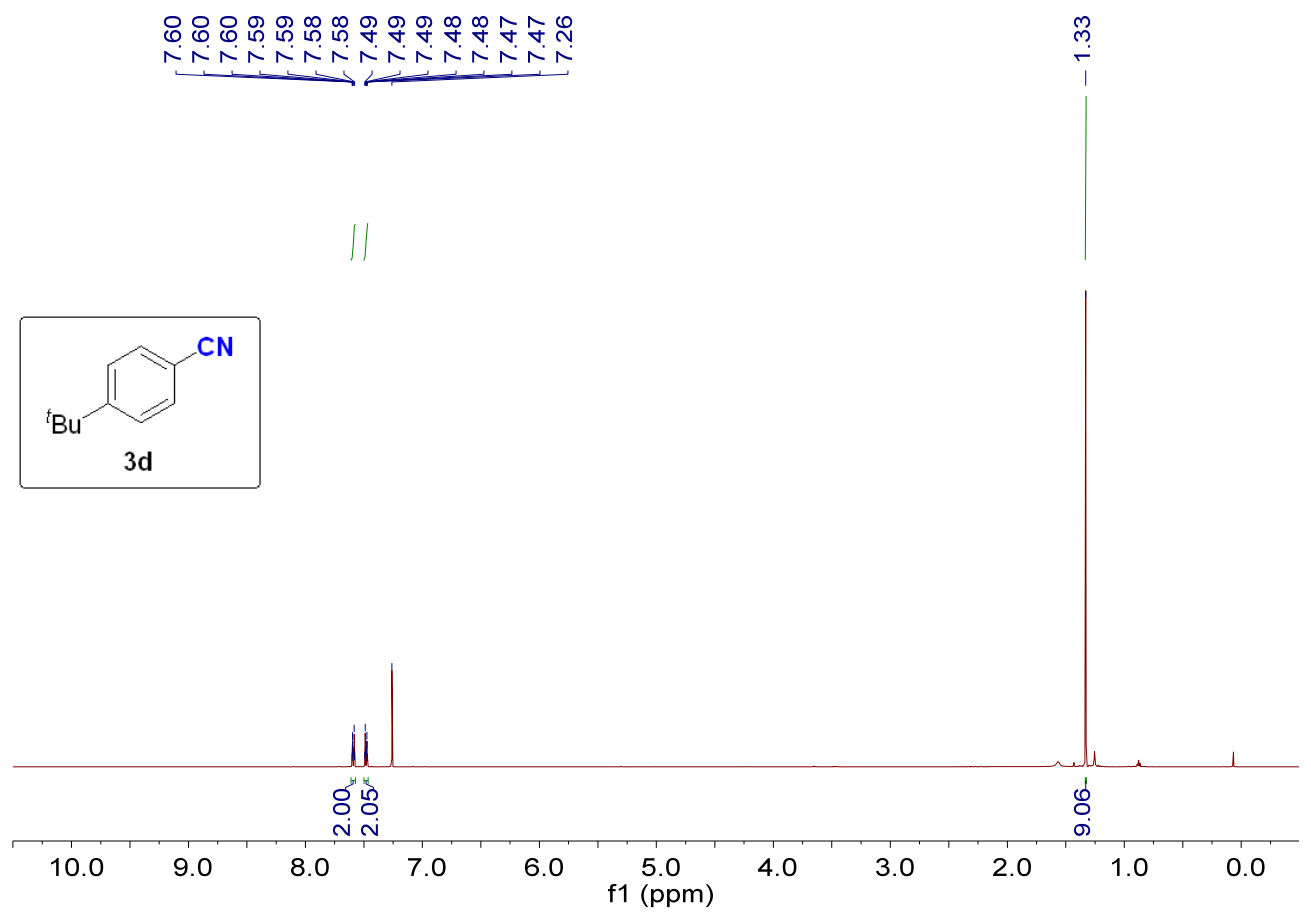

${ }^{1} \mathrm{H}$ NMR $(600 \mathrm{MHz})$ spectrum of $\mathbf{3 d}\left(\mathrm{CDCl}_{3}, \mathrm{rt}\right)$. 


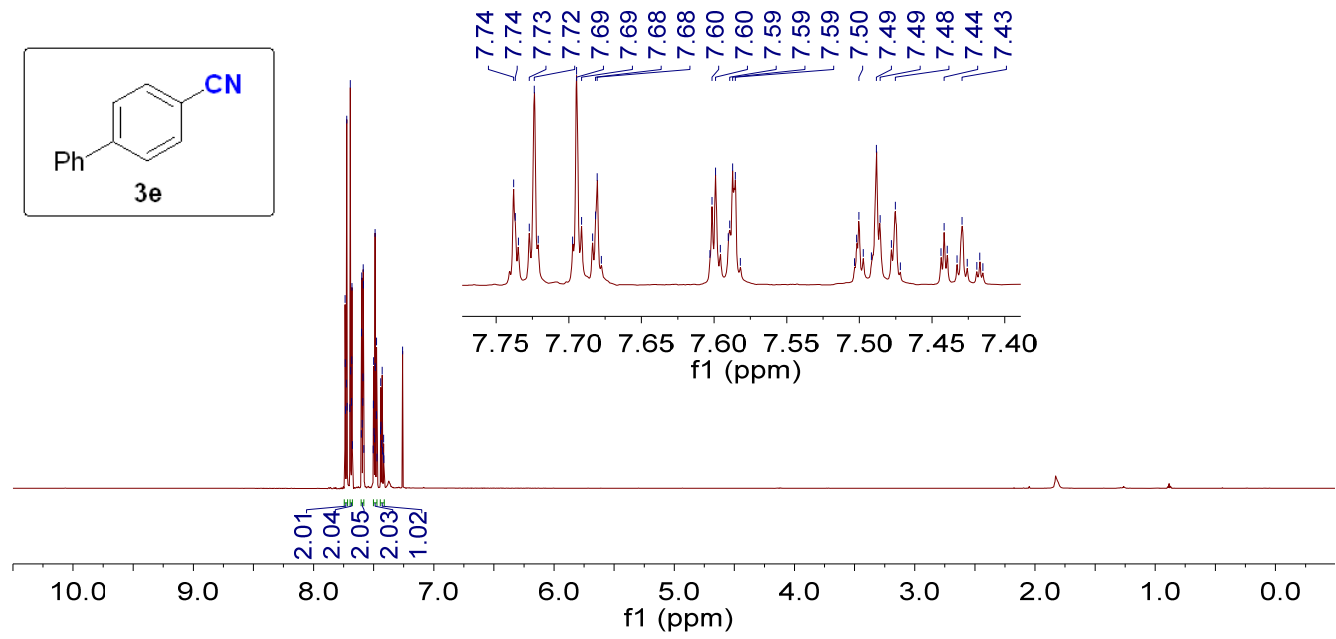

${ }^{1} \mathrm{H}$ NMR $(600 \mathrm{MHz})$ spectrum of $\mathbf{3 e}\left(\mathrm{CDCl}_{3}, \mathrm{rt}\right)$.

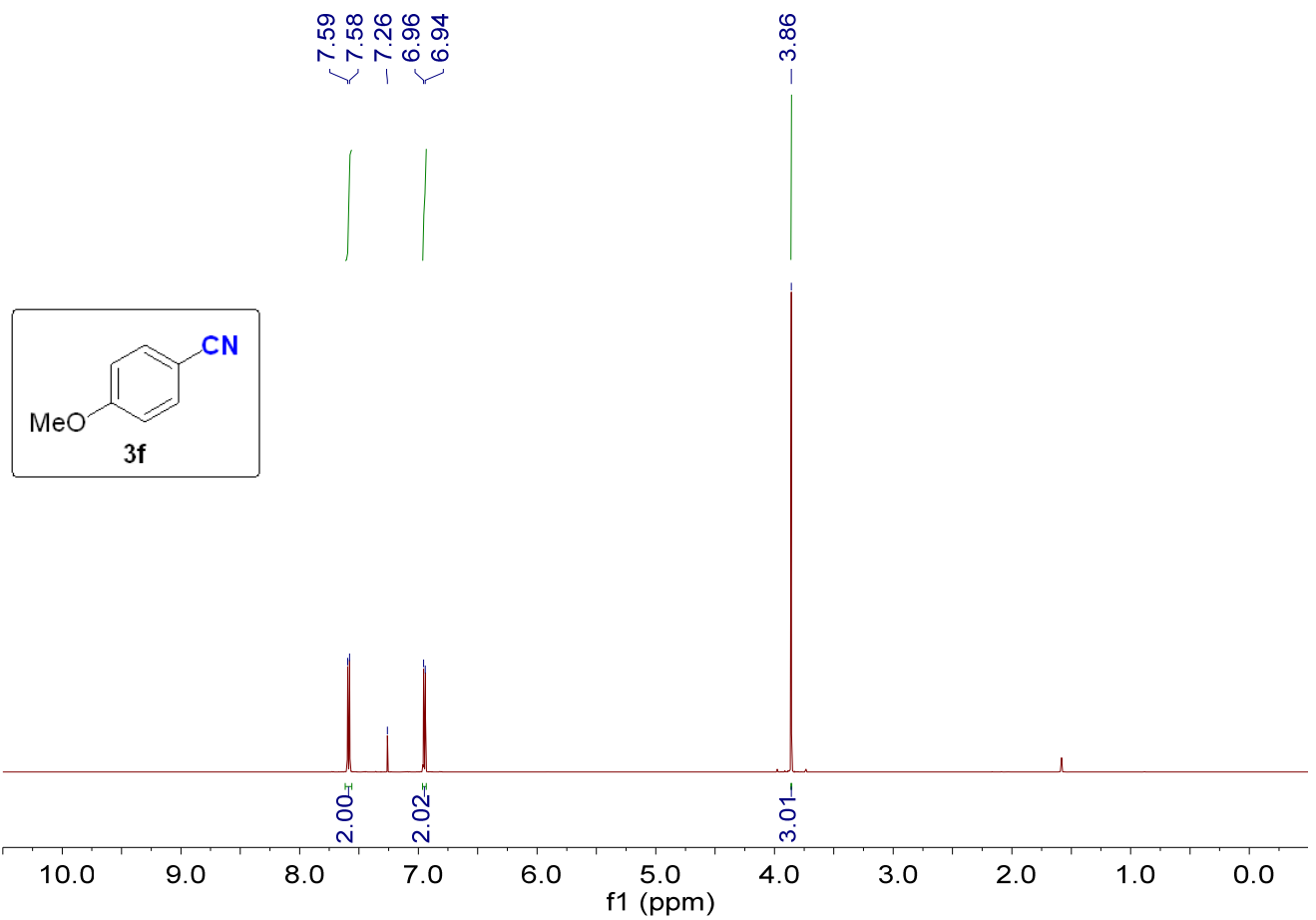

${ }^{1} \mathrm{H}$ NMR $(600 \mathrm{MHz})$ spectrum of $\mathbf{3 f}\left(\mathrm{CDCl}_{3}, \mathrm{rt}\right)$. 


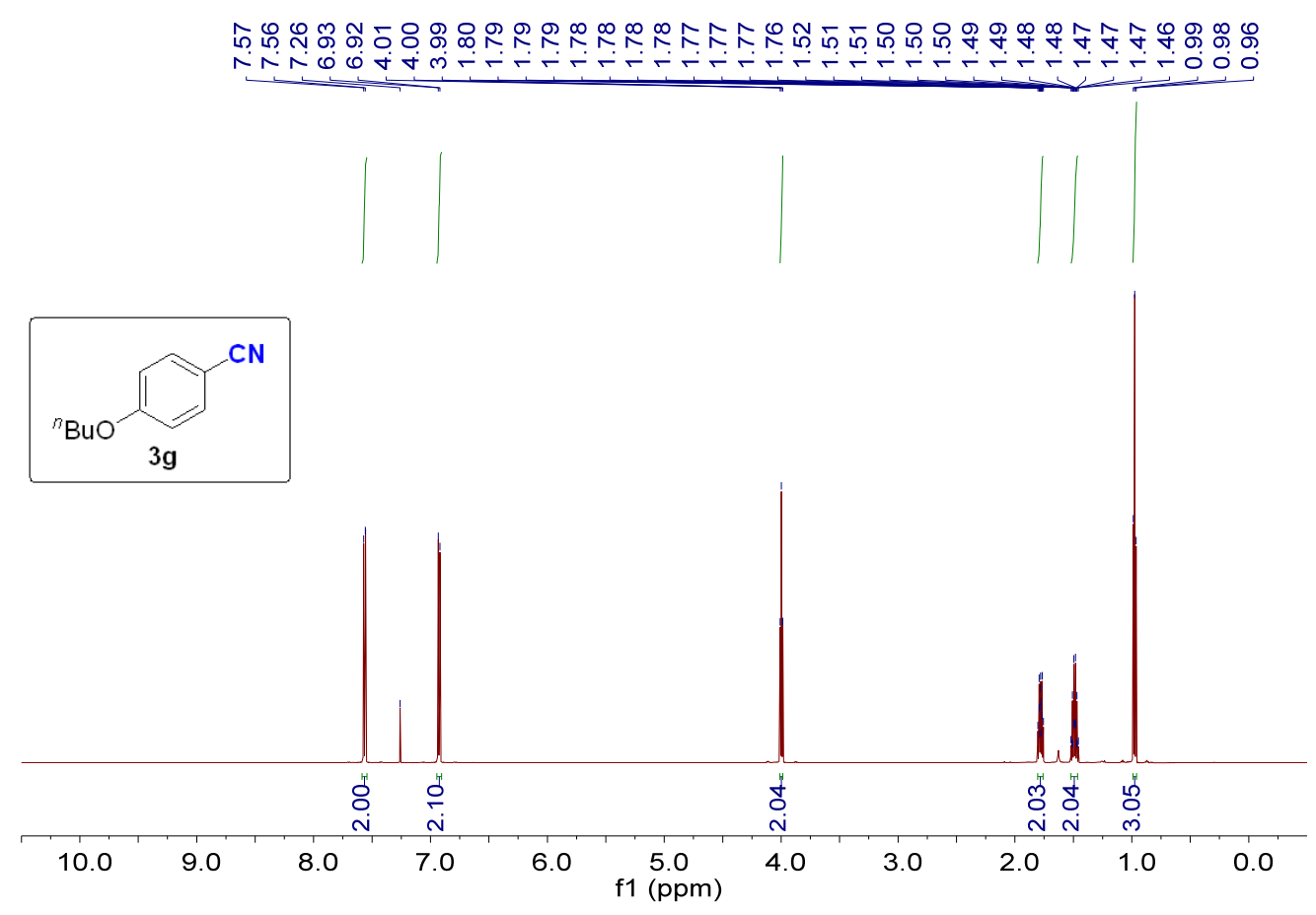

${ }^{1} \mathrm{H}$ NMR $(600 \mathrm{MHz})$ spectrum of $\mathbf{3 g}\left(\mathrm{CDCl}_{3}, \mathrm{rt}\right)$.

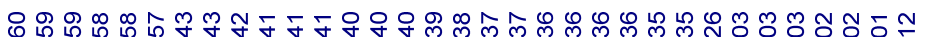

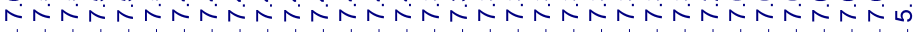
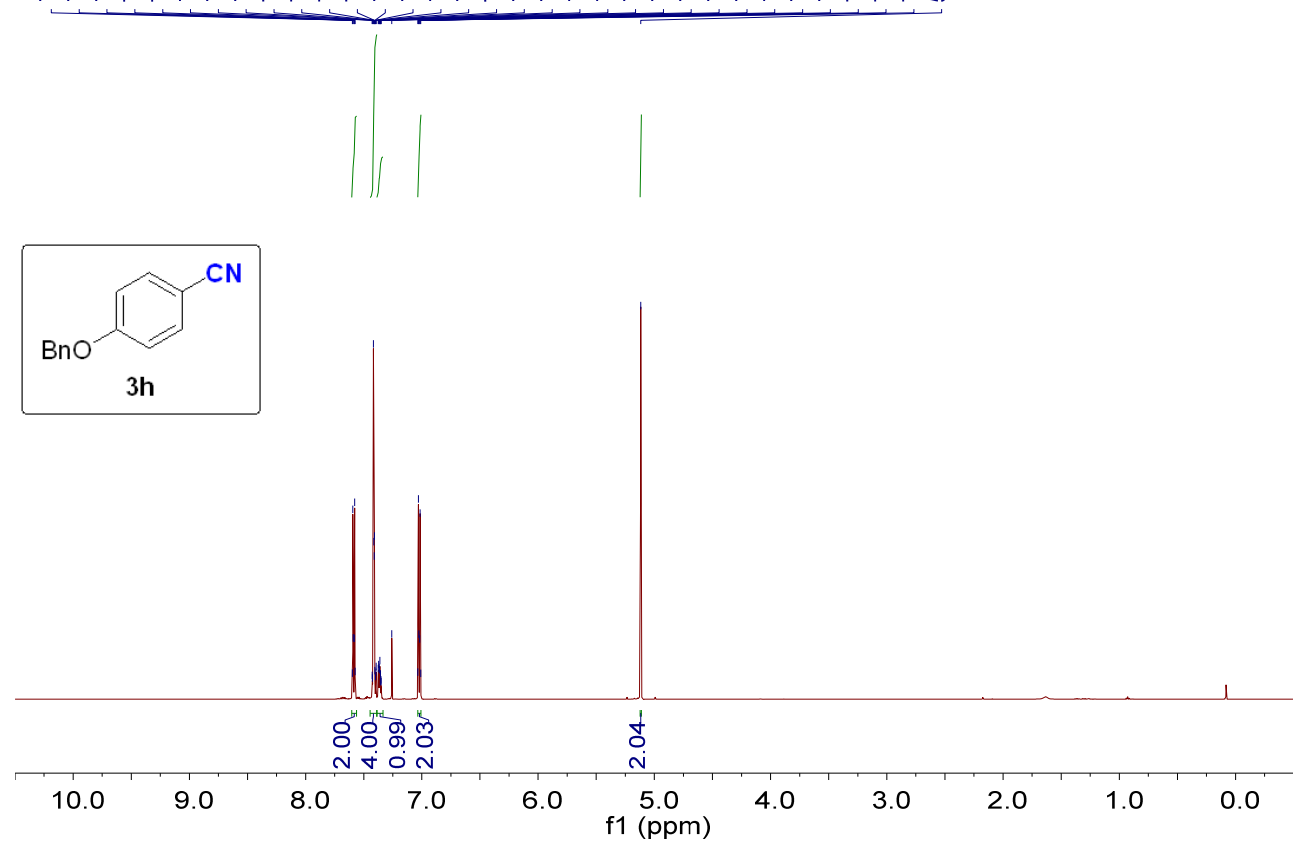

${ }^{1} \mathrm{H}$ NMR $(600 \mathrm{MHz})$ spectrum of $\mathbf{3 h}\left(\mathrm{CDCl}_{3}, \mathrm{rt}\right)$. 


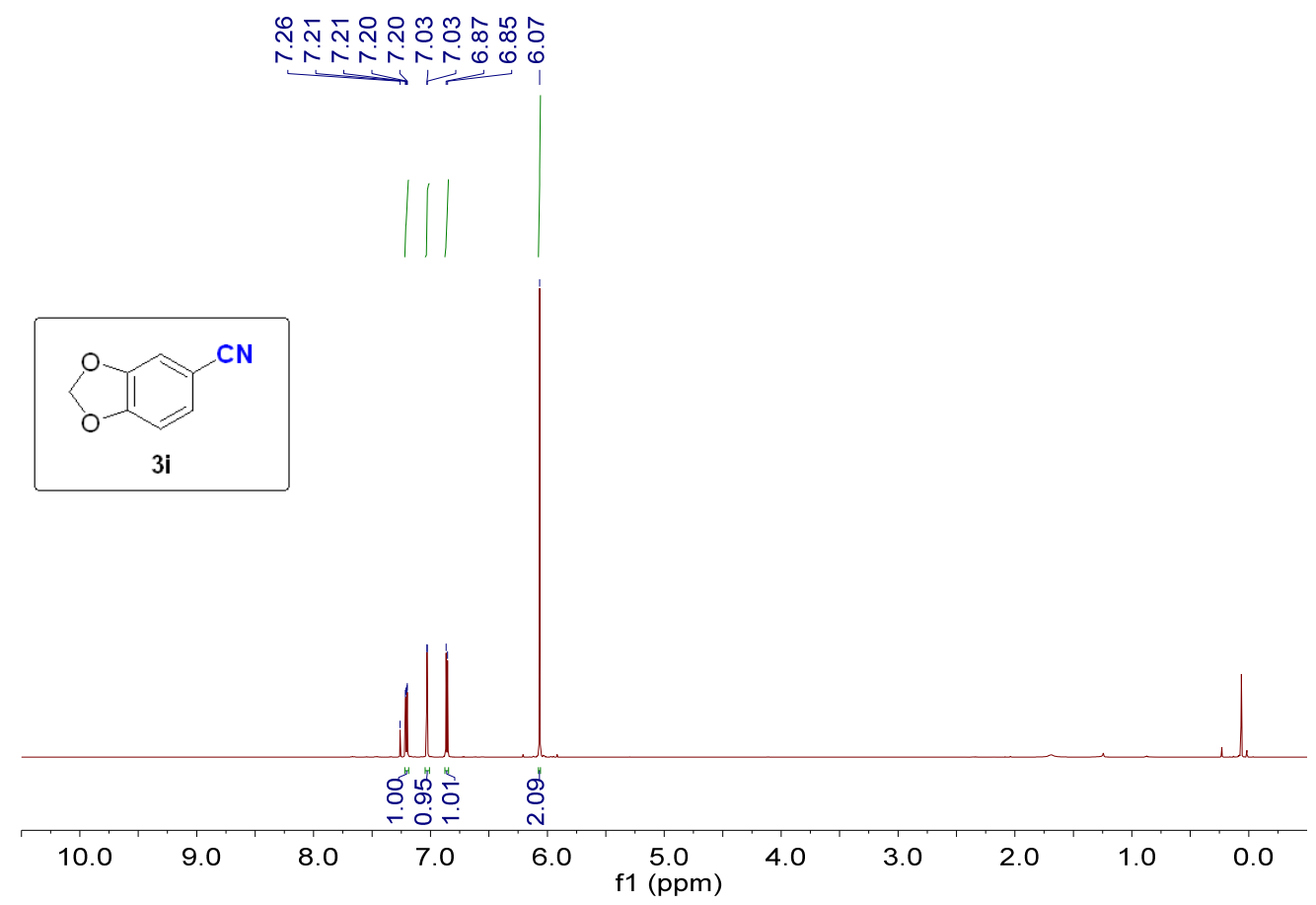

${ }^{1} \mathrm{H}$ NMR (600 MHz) spectrum of $\mathbf{3 i}\left(\mathrm{CDCl}_{3}, \mathrm{rt}\right)$.

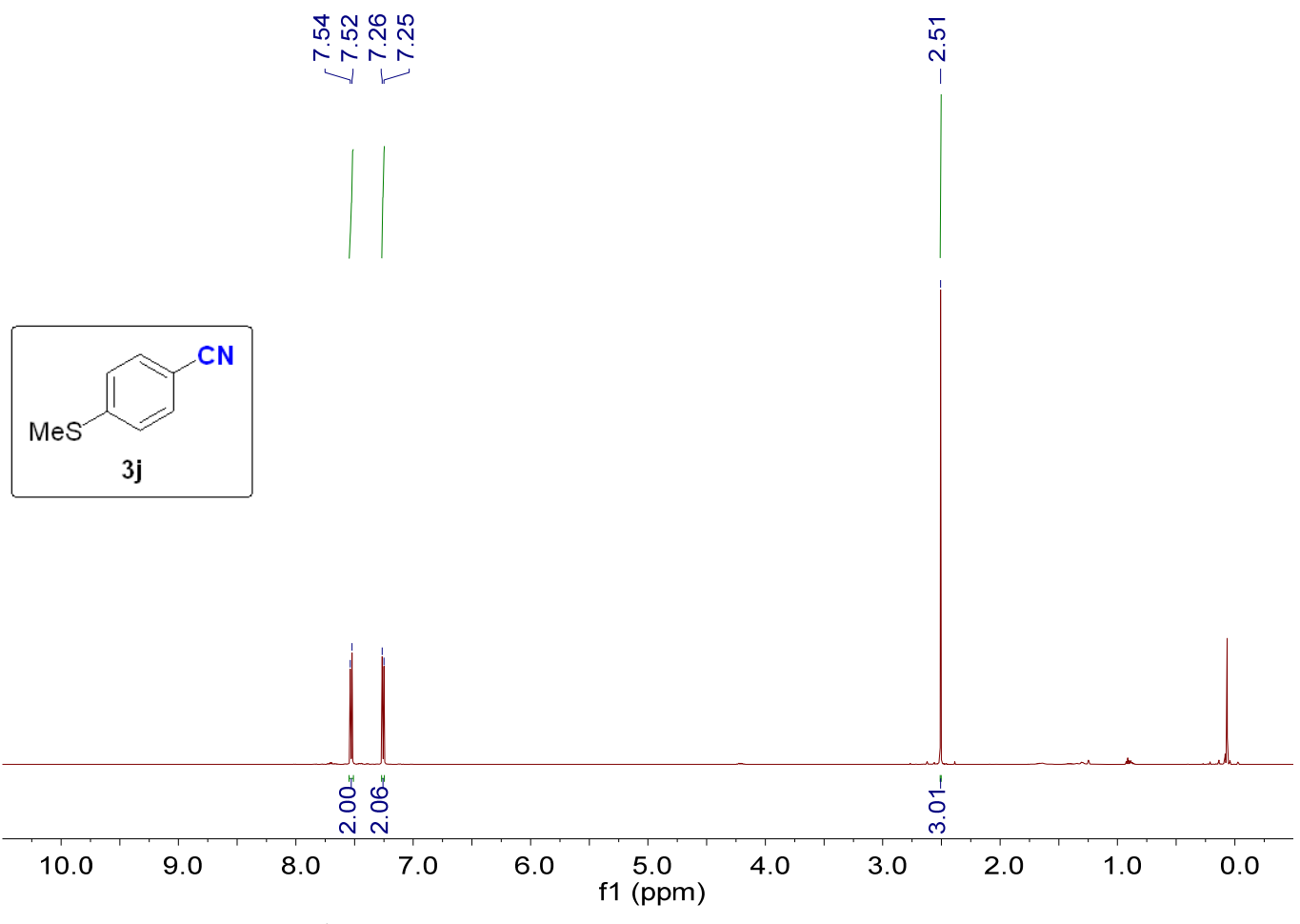

${ }^{1} \mathrm{H}$ NMR (600 MHz) spectrum of $\mathbf{3 j}\left(\mathrm{CDCl}_{3}\right.$, rt). 


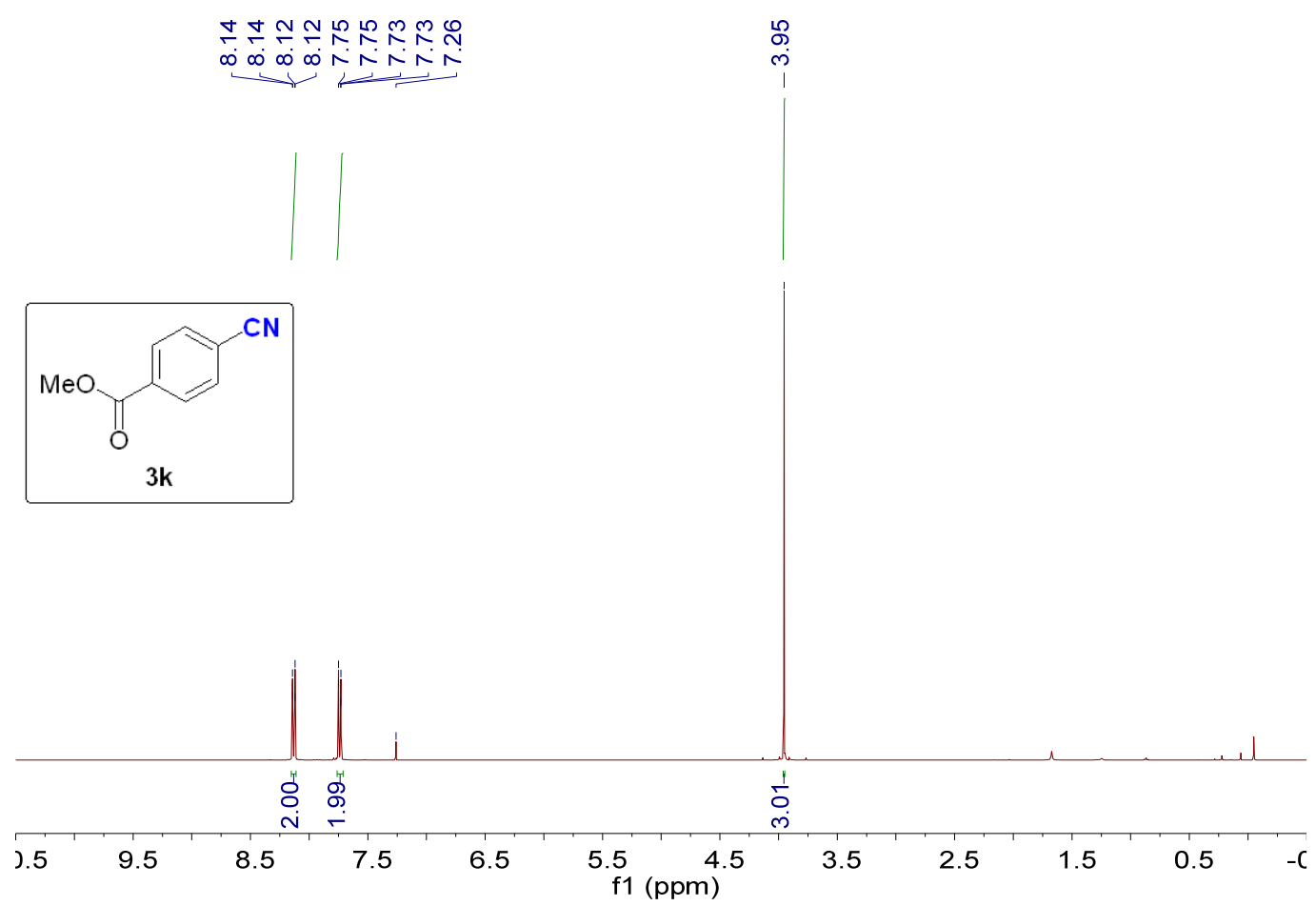

${ }^{1} \mathrm{H}$ NMR (400 MHz) spectrum of $\mathbf{3 k}\left(\mathrm{CDCl}_{3}, \mathrm{rt}\right)$.

బ్లై 실.

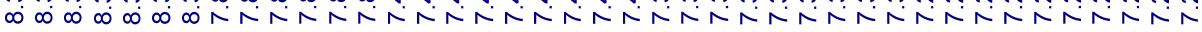

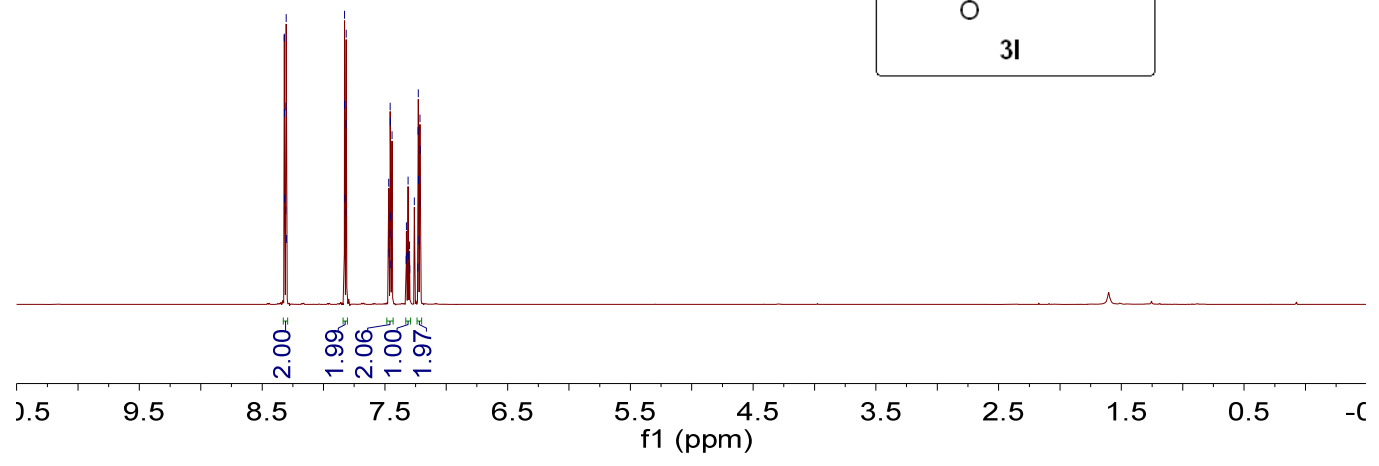

${ }^{1} \mathrm{H}$ NMR (600 MHz) spectrum of $\mathbf{3 I}\left(\mathrm{CDCl}_{3}, \mathrm{rt}\right)$. 


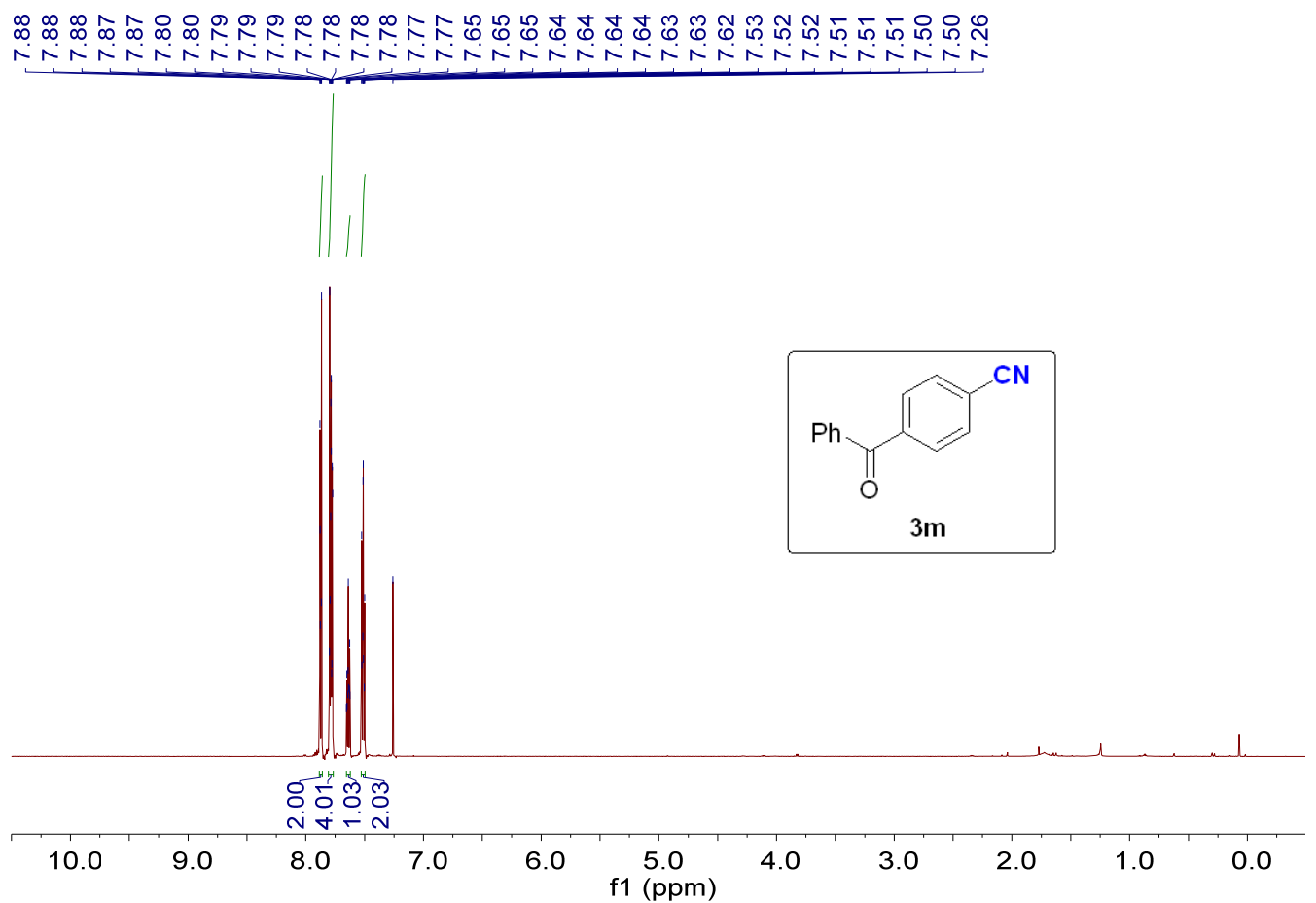

${ }^{1} \mathrm{H}$ NMR (600 MHz) spectrum of $\mathbf{3 m}\left(\mathrm{CDCl}_{3}\right.$, rt).

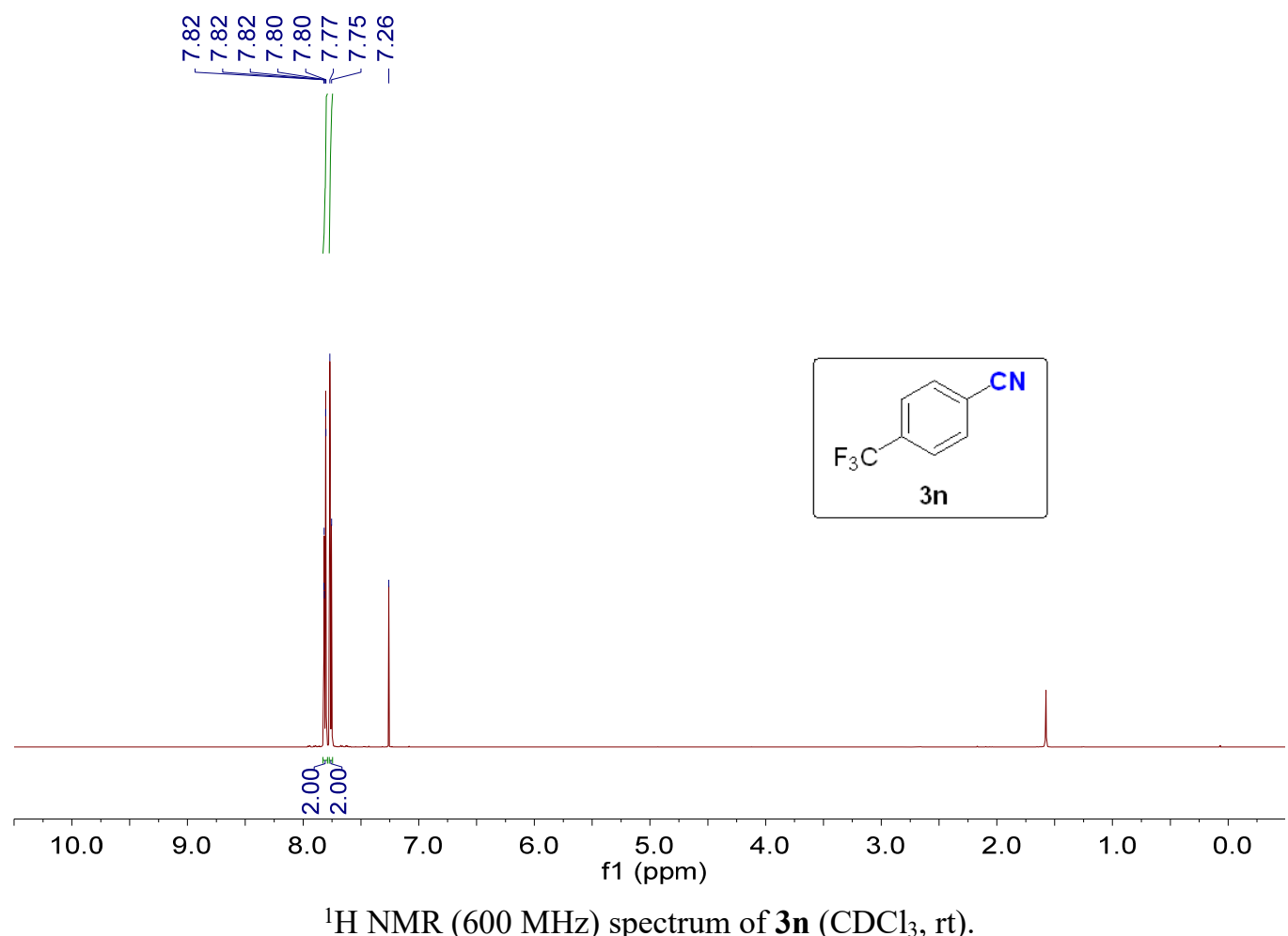




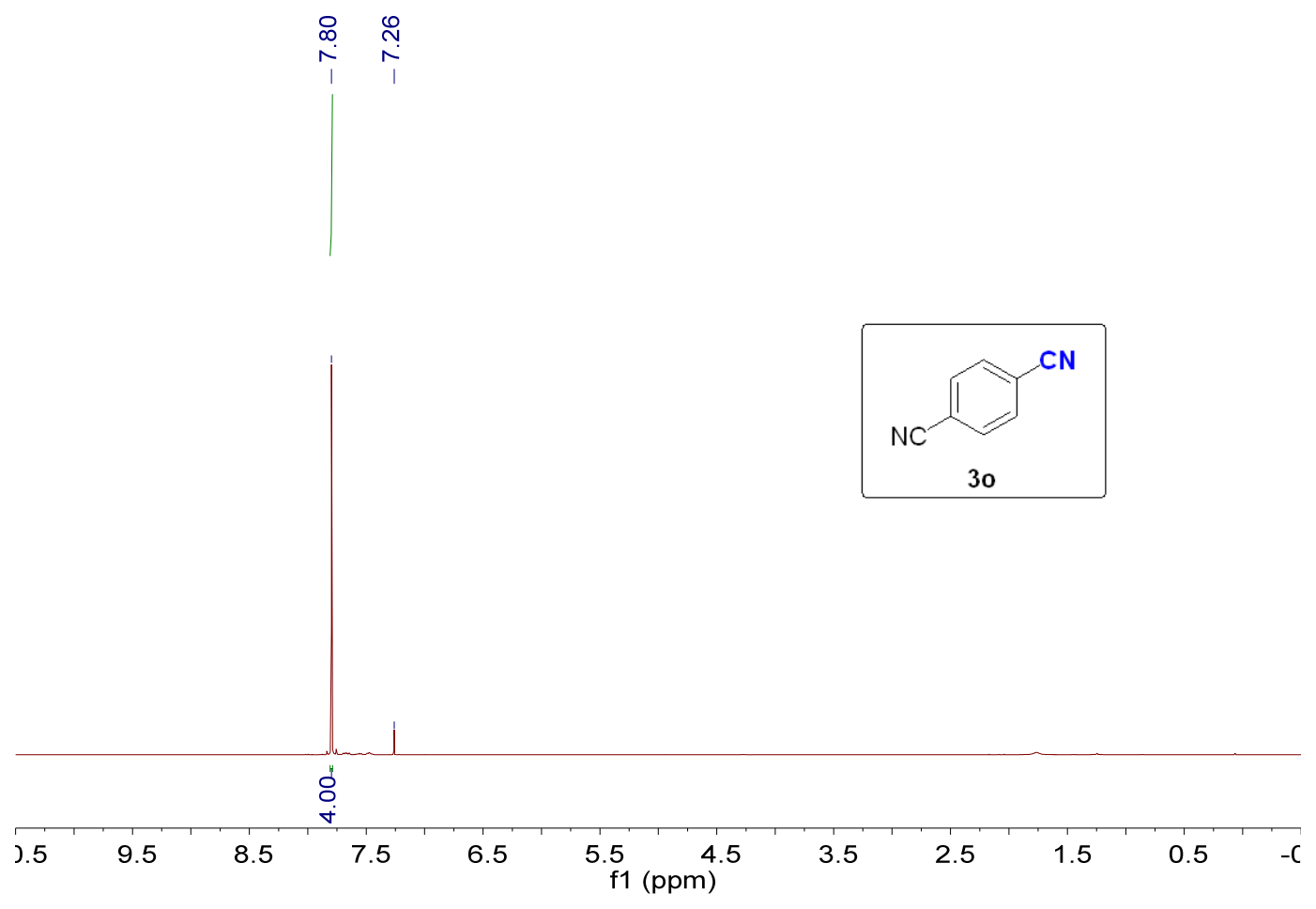

${ }^{1} \mathrm{H}$ NMR $(400 \mathrm{MHz})$ spectrum of $\mathbf{3 o}\left(\mathrm{CDCl}_{3}, \mathrm{rt}\right)$.

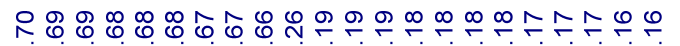

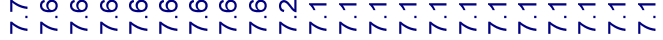

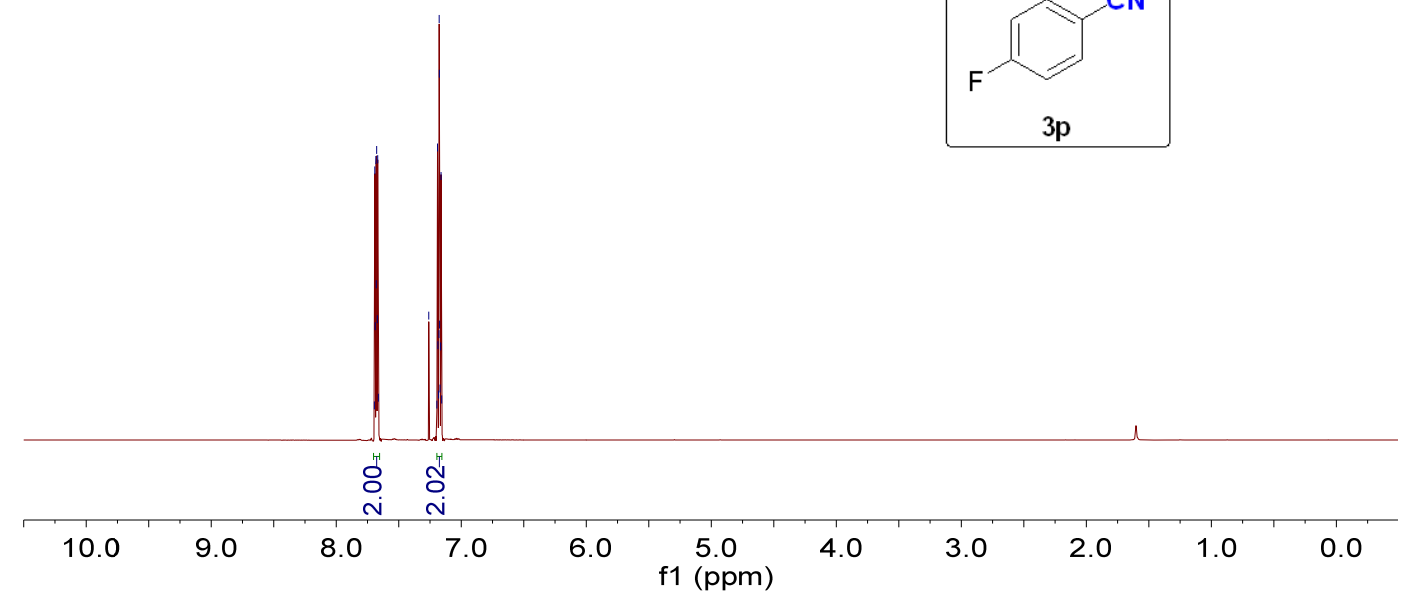

${ }^{1} \mathrm{H}$ NMR $(600 \mathrm{MHz})$ spectrum of $\mathbf{3 p}\left(\mathrm{CDCl}_{3}, \mathrm{rt}\right)$. 


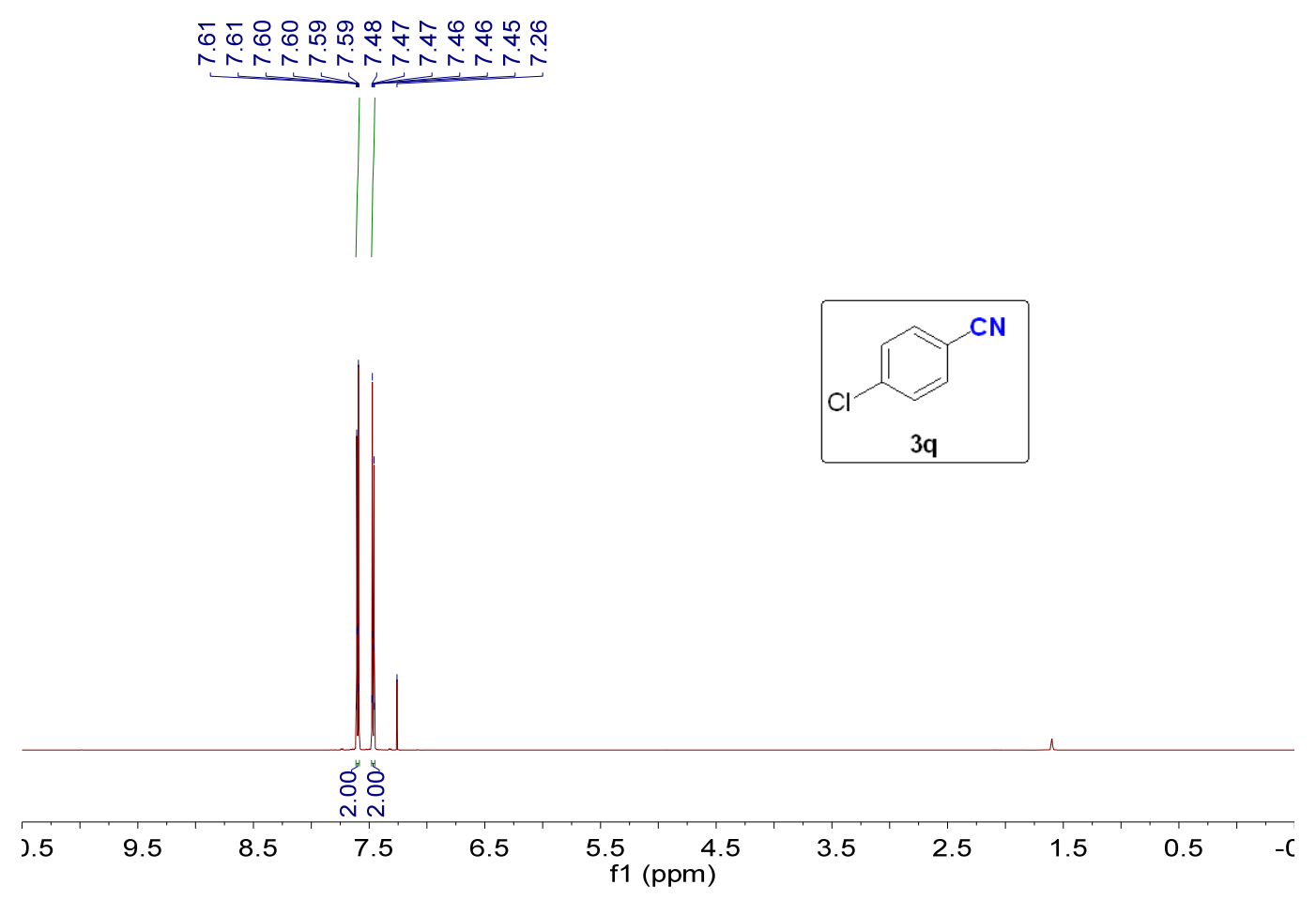

${ }^{1} \mathrm{H}$ NMR (600 MHz) spectrum of $\mathbf{3 q}\left(\mathrm{CDCl}_{3}, \mathrm{rt}\right)$.

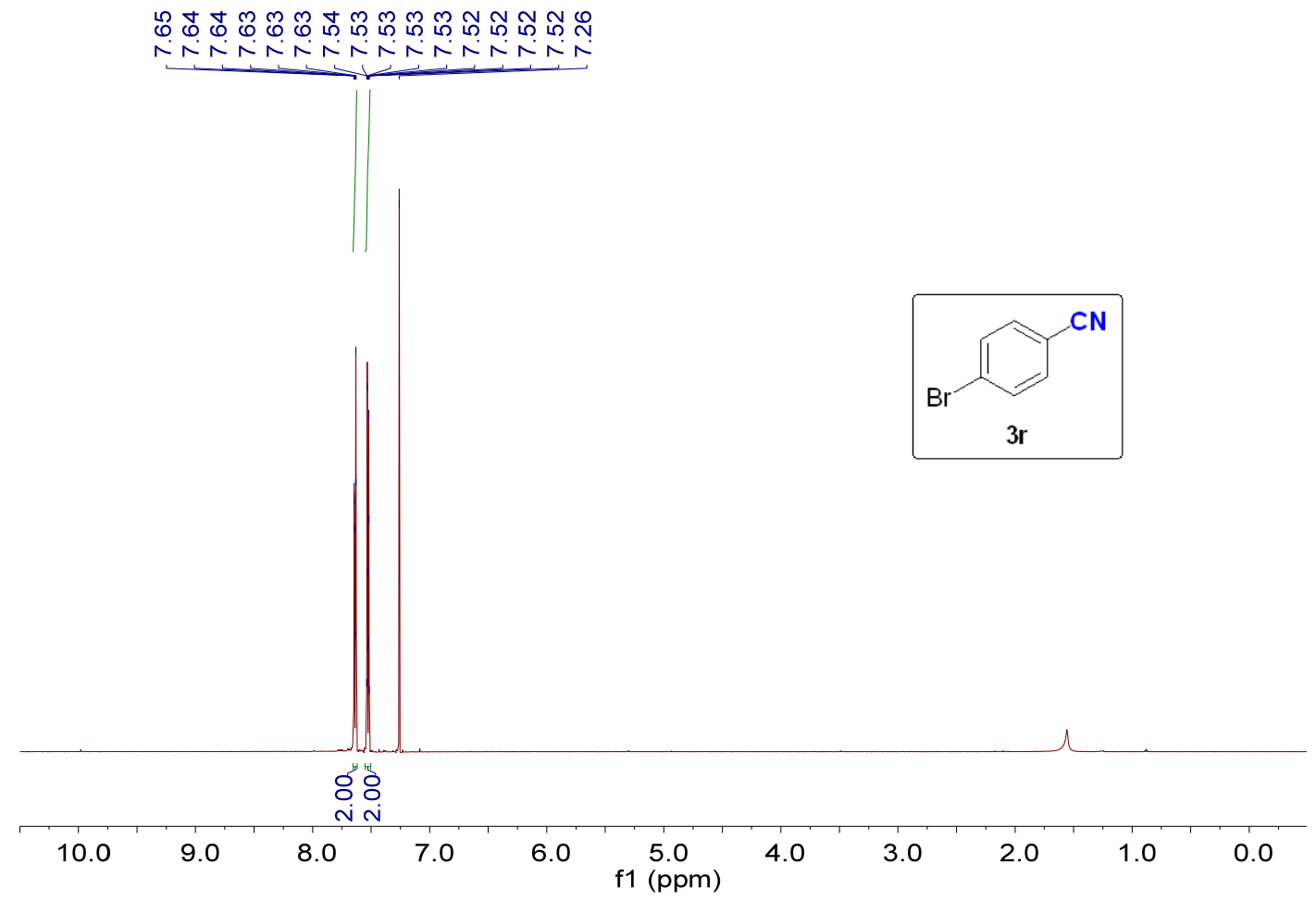

${ }^{1} \mathrm{H}$ NMR $(600 \mathrm{MHz})$ spectrum of $3 \mathbf{r}\left(\mathrm{CDCl}_{3}, \mathrm{rt}\right)$. 


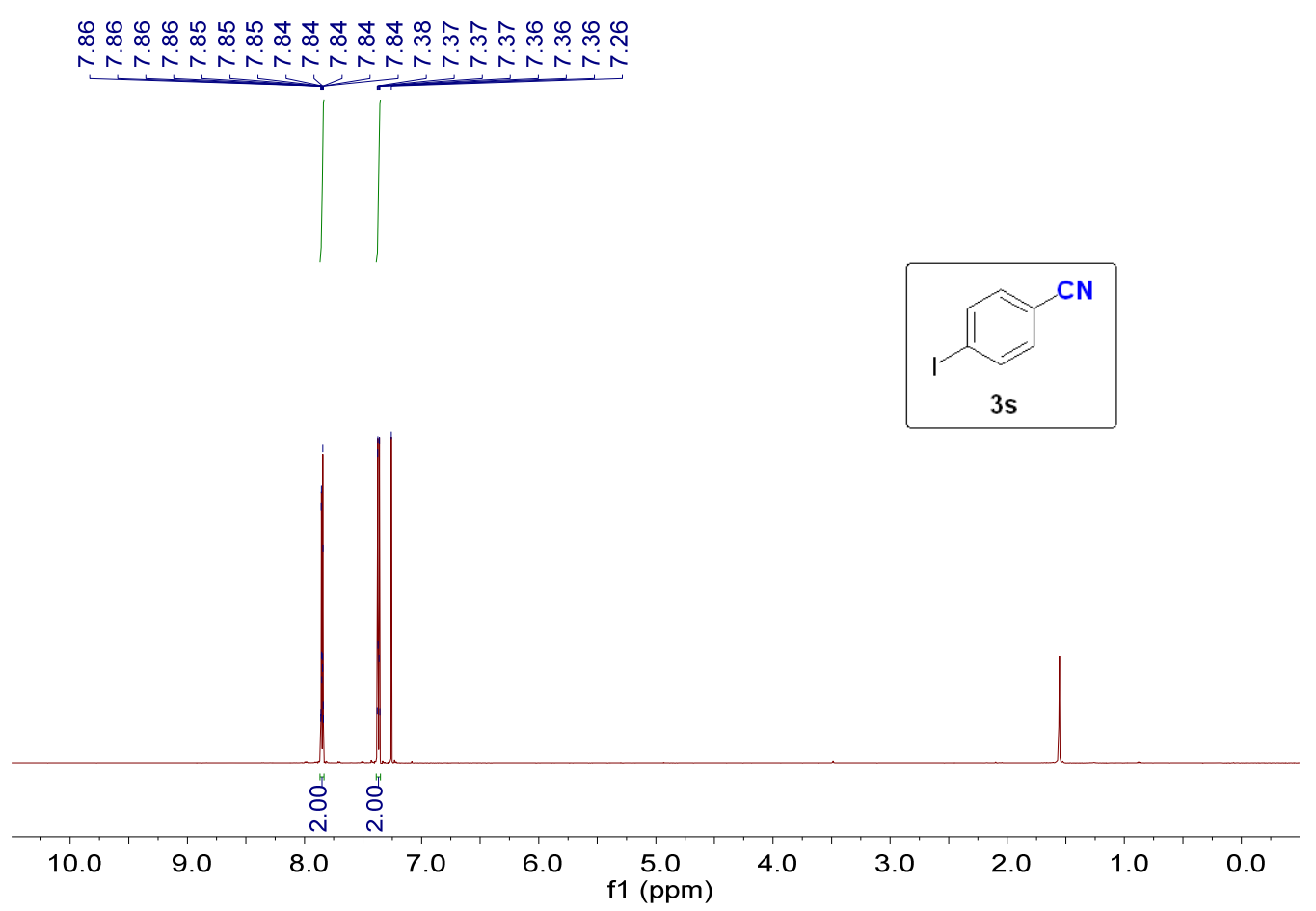

${ }^{1} \mathrm{H}$ NMR $(600 \mathrm{MHz})$ spectrum of $\mathbf{3 s}\left(\mathrm{CDCl}_{3}, \mathrm{rt}\right)$.

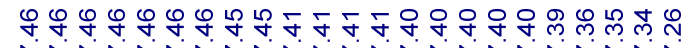

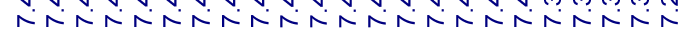

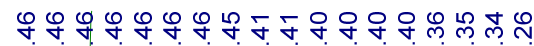

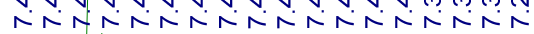
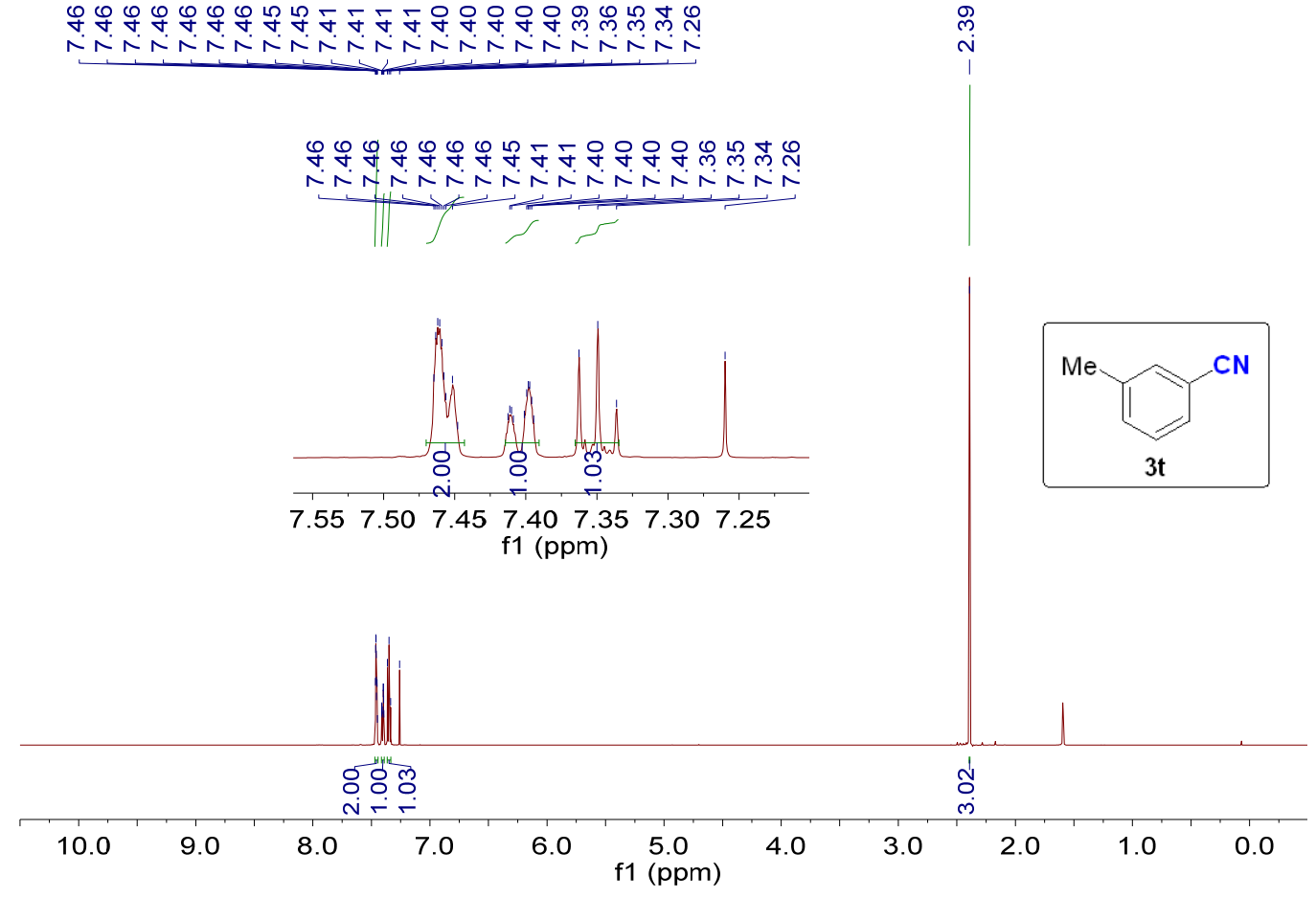

${ }^{1} \mathrm{H}$ NMR $(600 \mathrm{MHz})$ spectrum of $\mathbf{3 t}\left(\mathrm{CDCl}_{3}, \mathrm{rt}\right)$. 

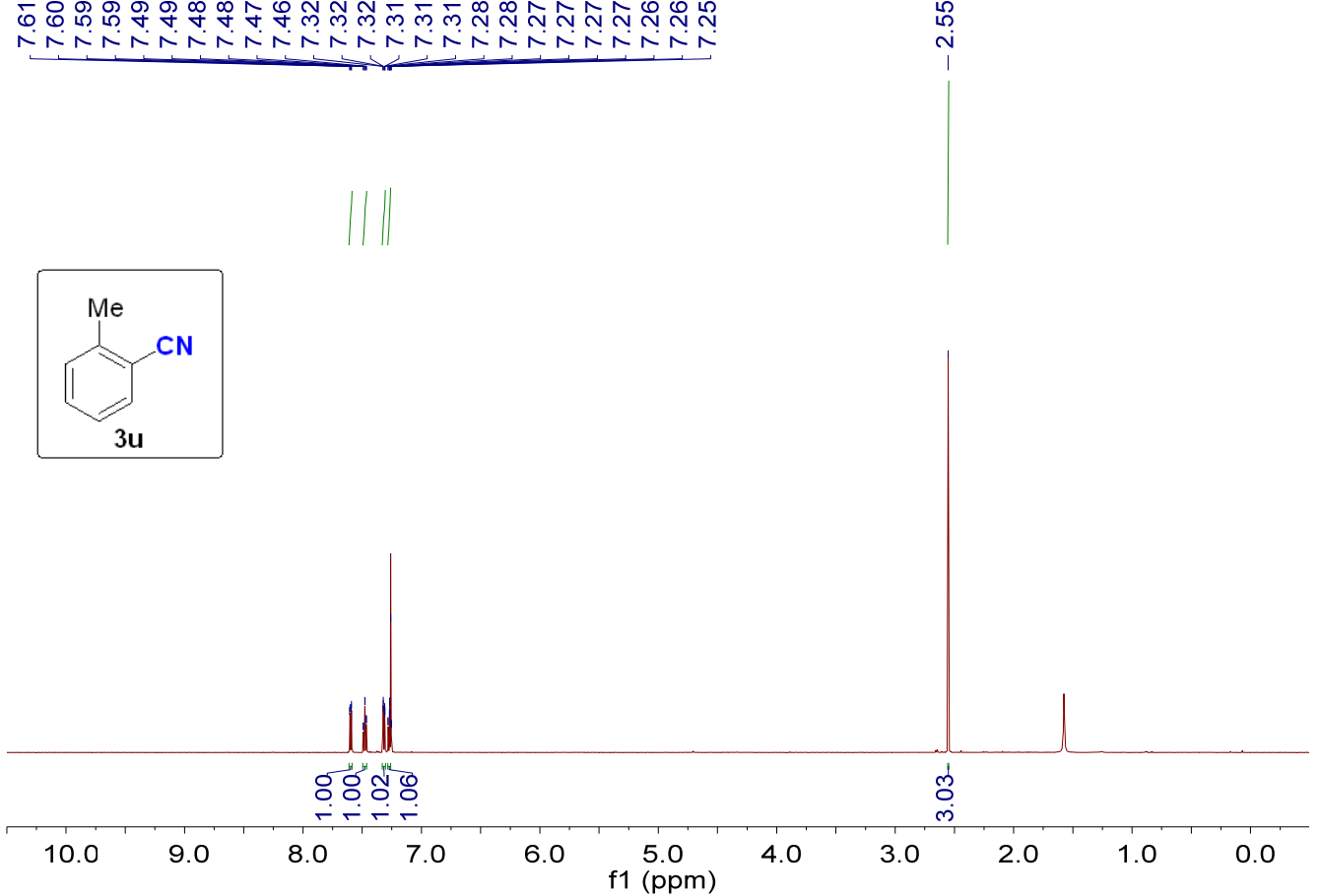

${ }^{1} \mathrm{H}$ NMR $(600 \mathrm{MHz})$ spectrum of $3 \mathbf{u}\left(\mathrm{CDCl}_{3}, \mathrm{rt}\right)$.

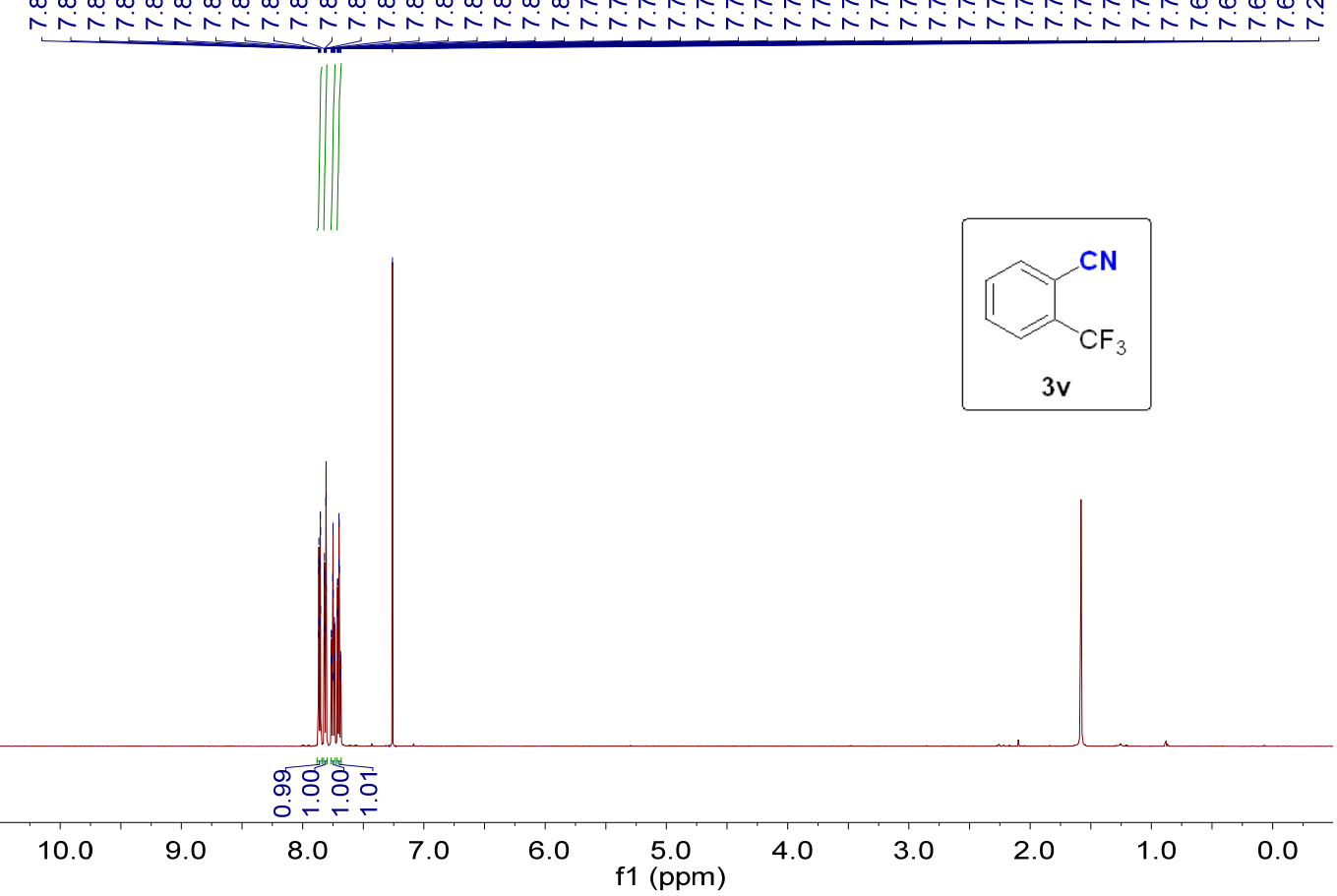

${ }^{1} \mathrm{H}$ NMR (600 MHz) spectrum of $\mathbf{3 v}\left(\mathrm{CDCl}_{3}, \mathrm{rt}\right)$. 

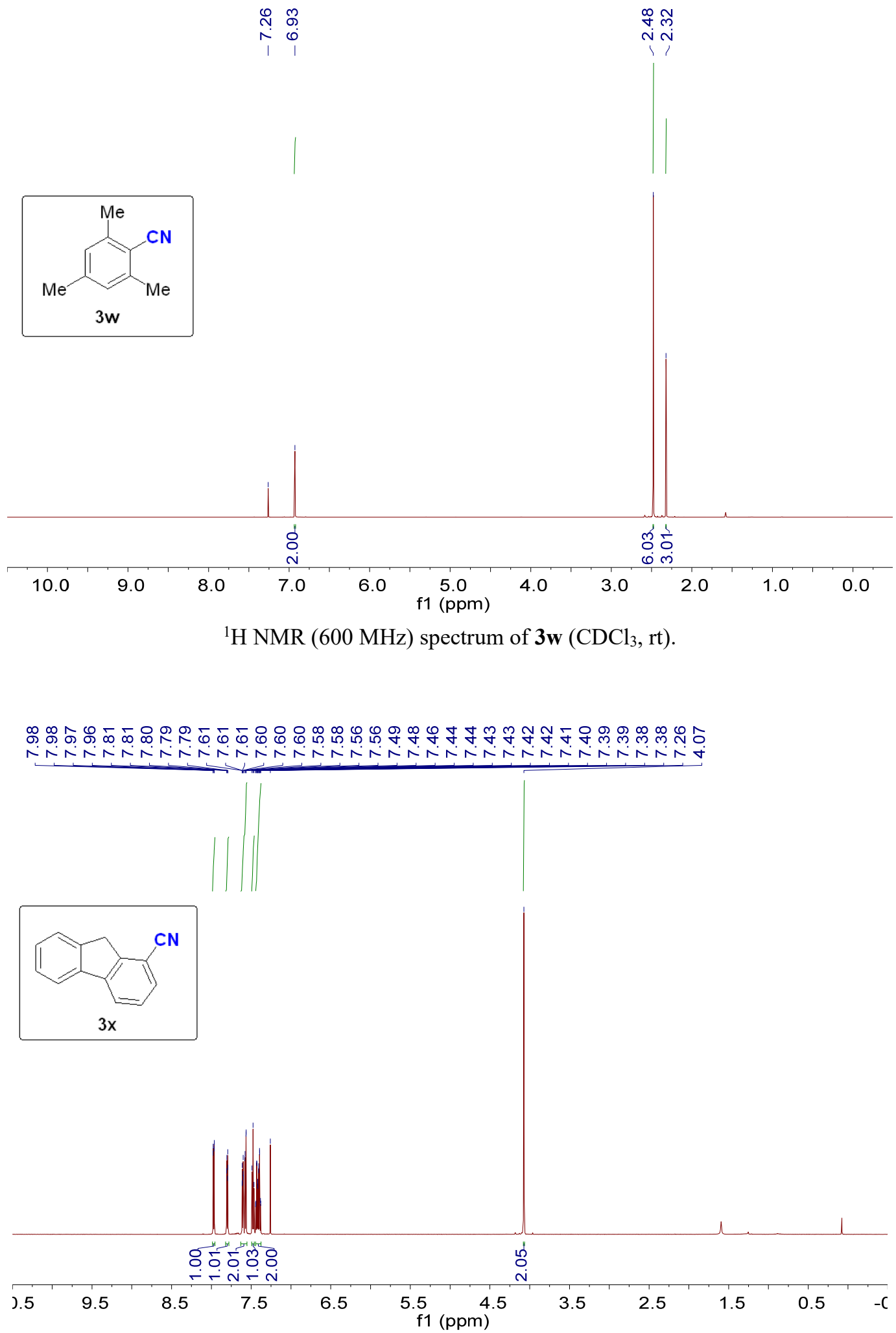

${ }^{1} \mathrm{H}$ NMR $(600 \mathrm{MHz})$ spectrum of $\mathbf{3} \mathbf{x}\left(\mathrm{CDCl}_{3}, \mathrm{rt}\right)$. 


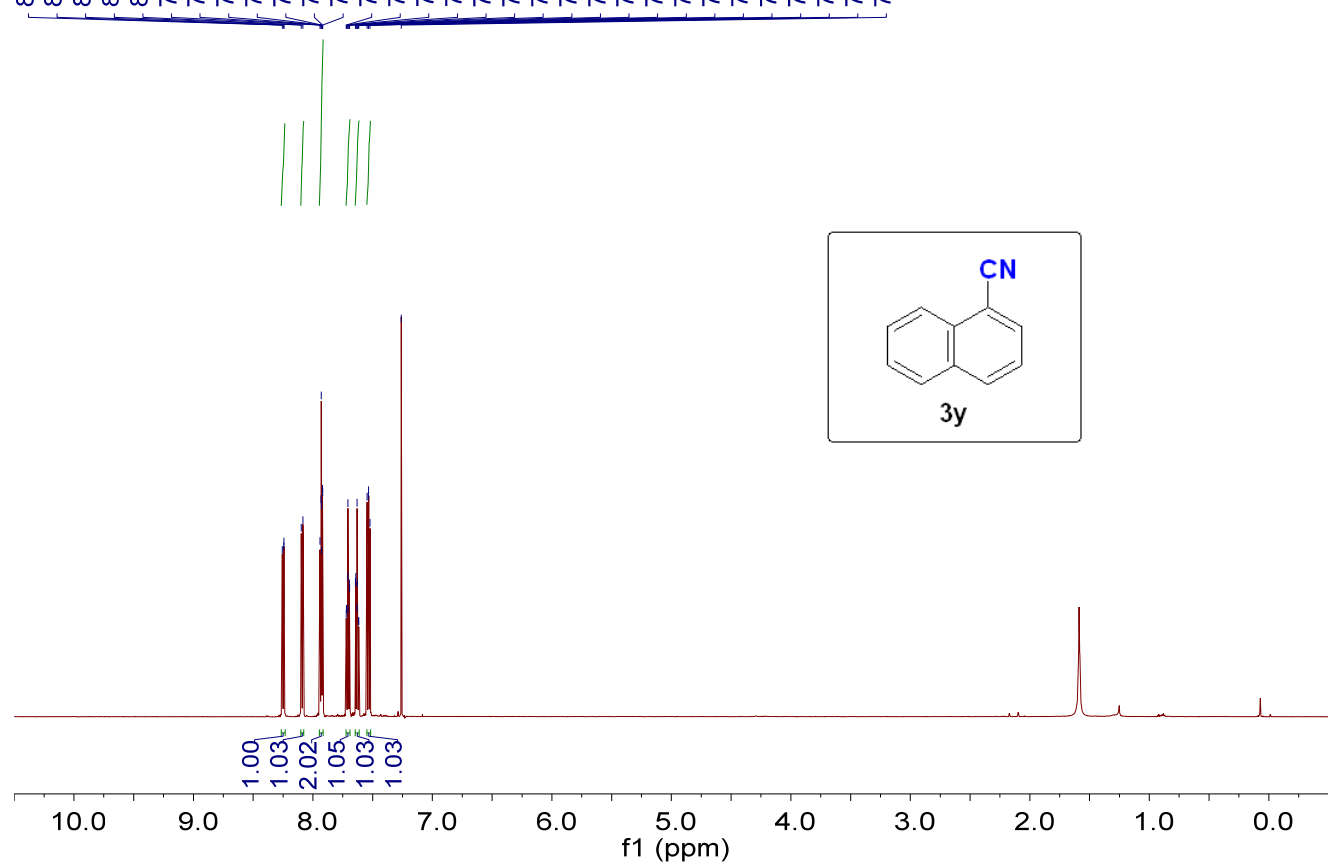

${ }^{1} \mathrm{H}$ NMR $(600 \mathrm{MHz})$ spectrum of $\mathbf{3 y}\left(\mathrm{CDCl}_{3}, \mathrm{rt}\right)$.

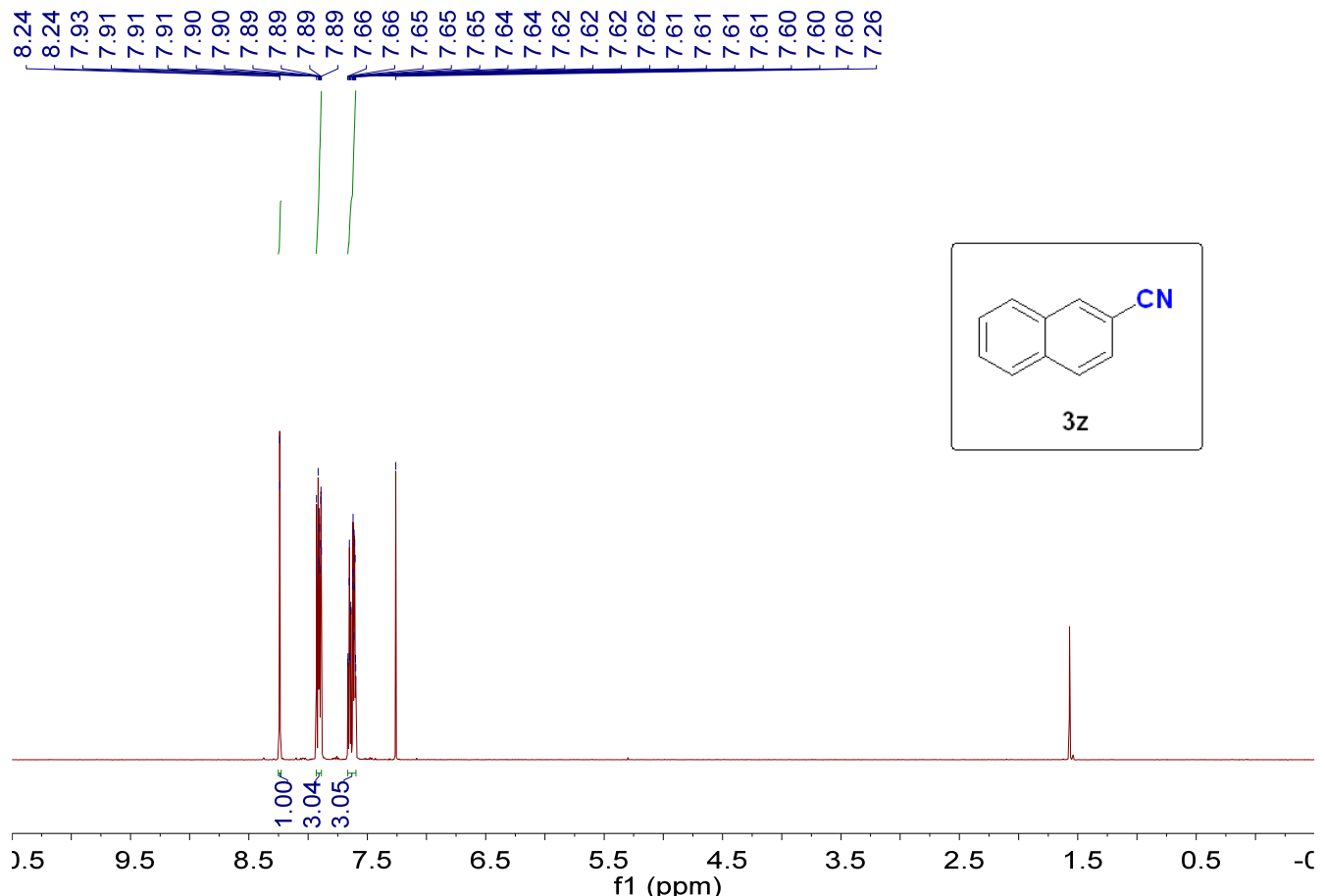

${ }^{1} \mathrm{H}$ NMR $(600 \mathrm{MHz})$ spectrum of $\mathbf{3 z}\left(\mathrm{CDCl}_{3}, \mathrm{rt}\right)$. 


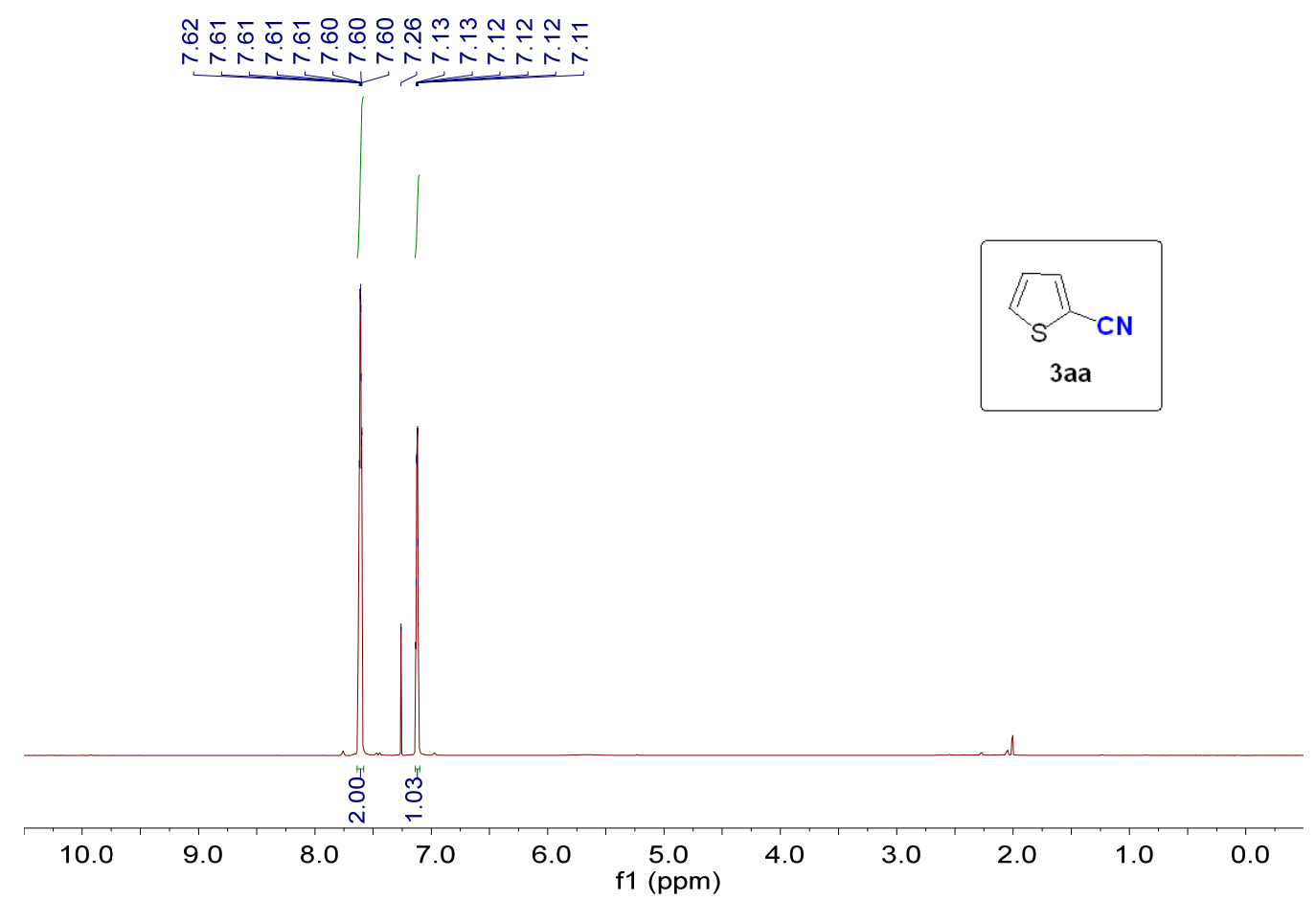

${ }^{1} \mathrm{H}$ NMR (400 MHz) spectrum of $\mathbf{3 a a}\left(\mathrm{CDCl}_{3}, \mathrm{rt}\right)$.

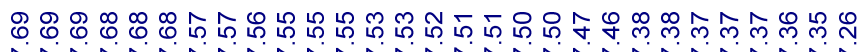

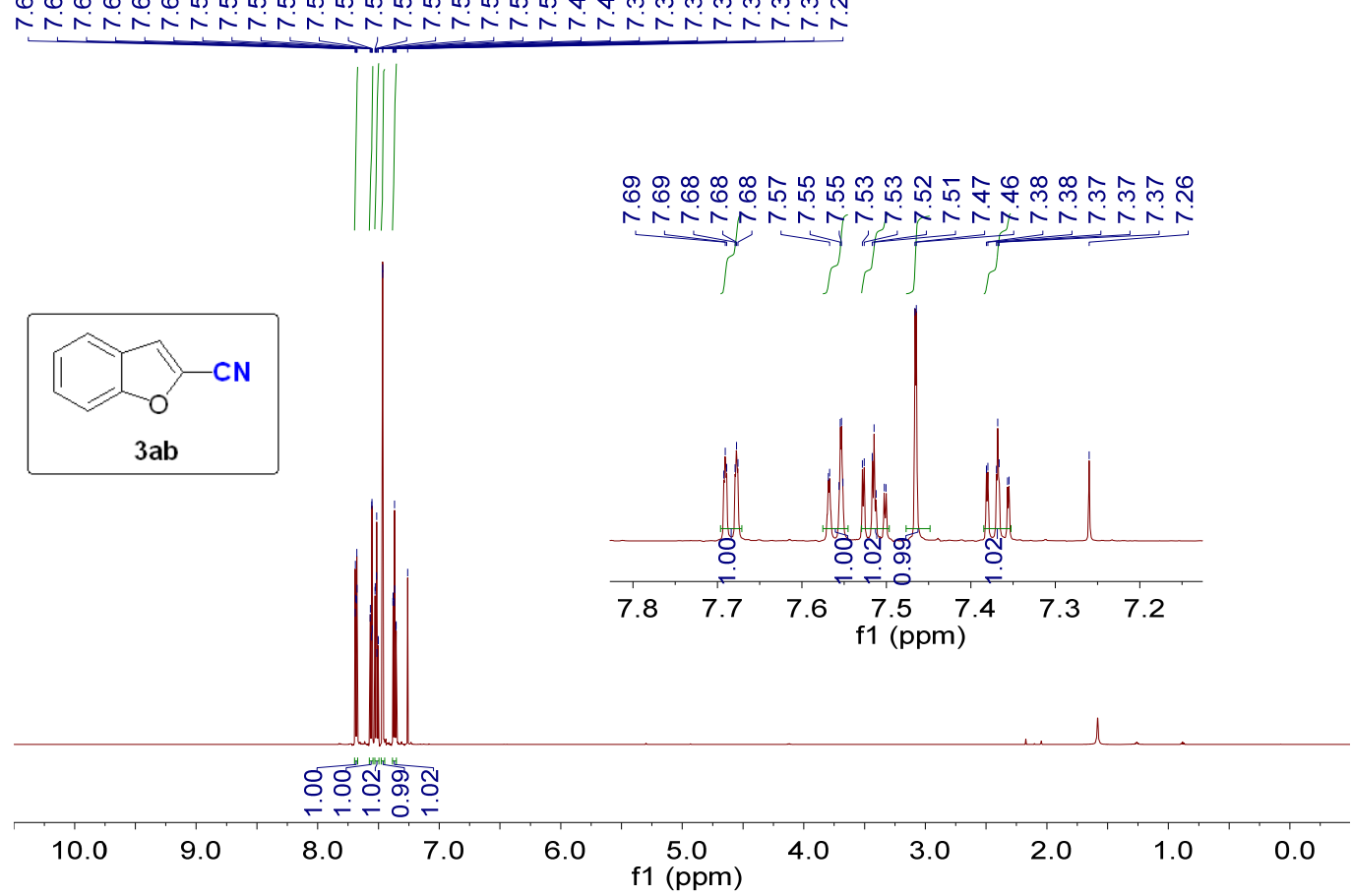

${ }^{1} \mathrm{H}$ NMR $(600 \mathrm{MHz})$ spectrum of $\mathbf{3 a b}\left(\mathrm{CDCl}_{3}, \mathrm{rt}\right)$. 
운 움

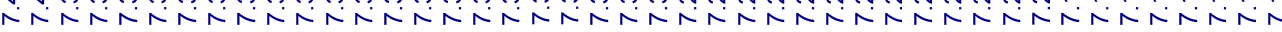
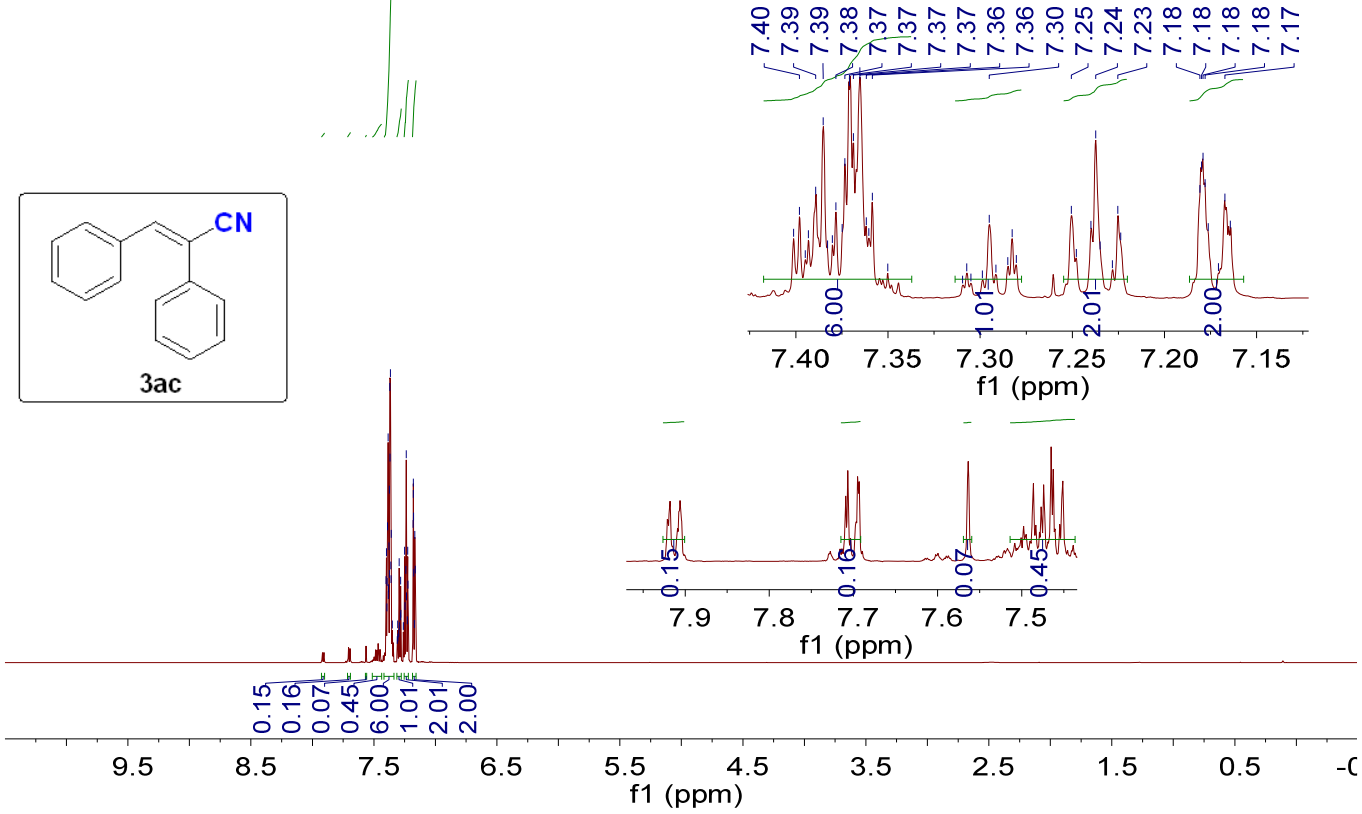

${ }^{1} \mathrm{H}$ NMR (600 MHz) spectrum of $\mathbf{3 a c}\left(\mathrm{CDCl}_{3}\right.$, rt).

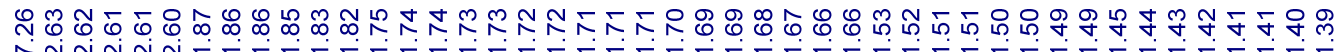

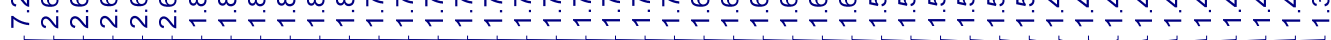

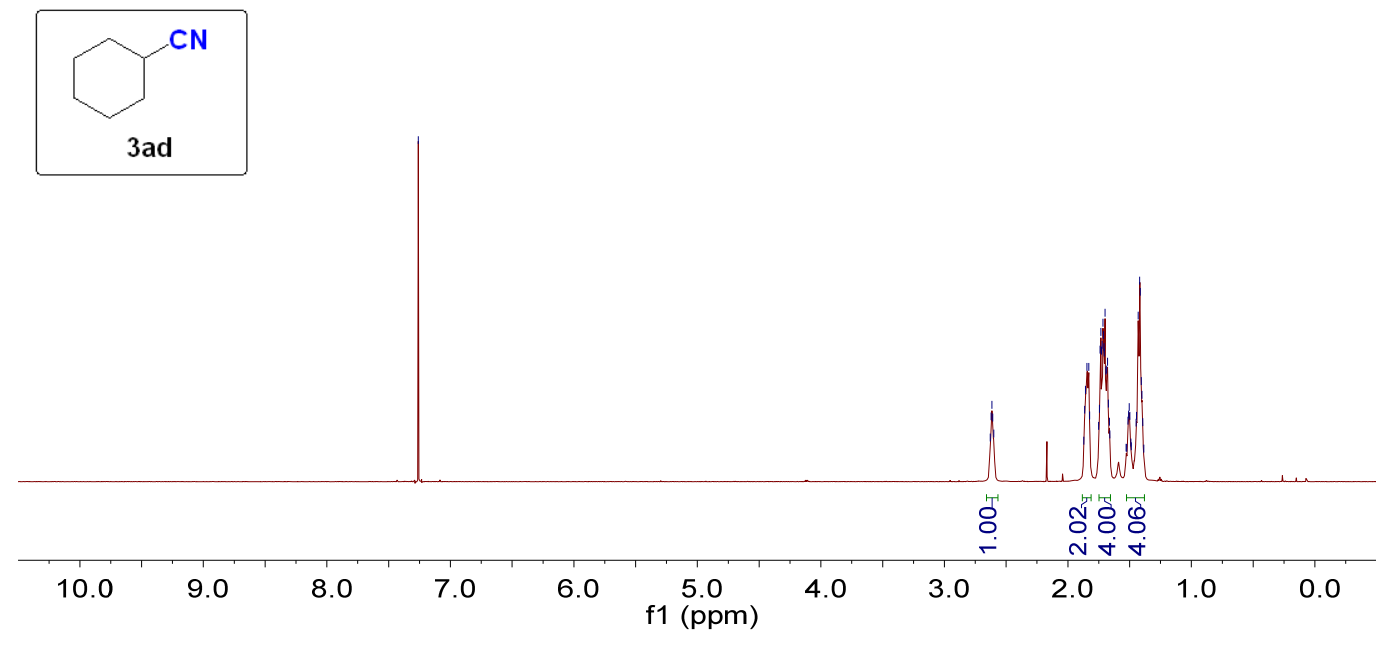

${ }^{1} \mathrm{H}$ NMR $(600 \mathrm{MHz})$ spectrum of $\mathbf{3 a d}\left(\mathrm{CDCl}_{3}, \mathrm{rt}\right)$. 


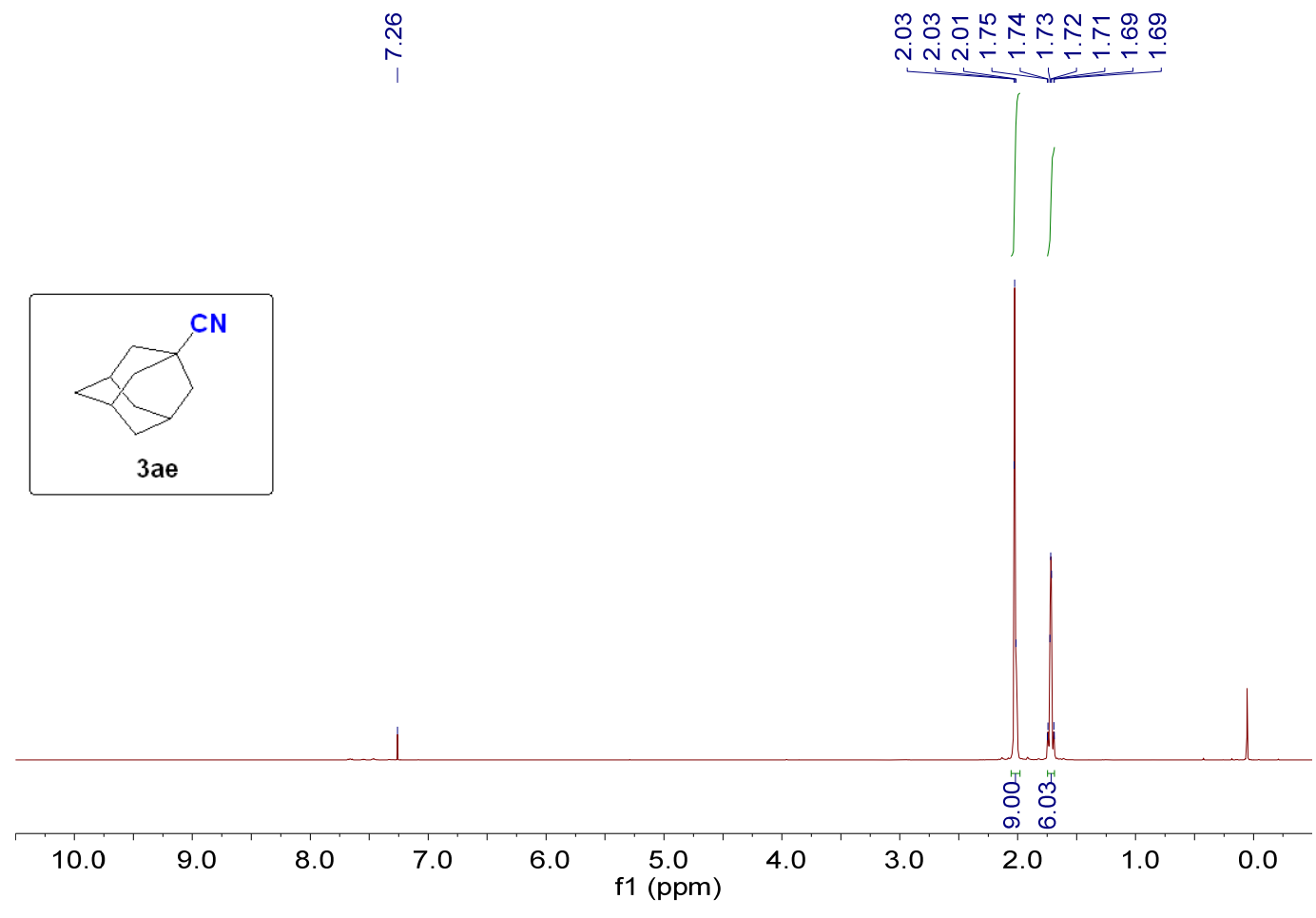

${ }^{1} \mathrm{H}$ NMR $(600 \mathrm{MHz})$ spectrum of $\mathbf{3 a e}\left(\mathrm{CDCl}_{3}\right.$, rt).

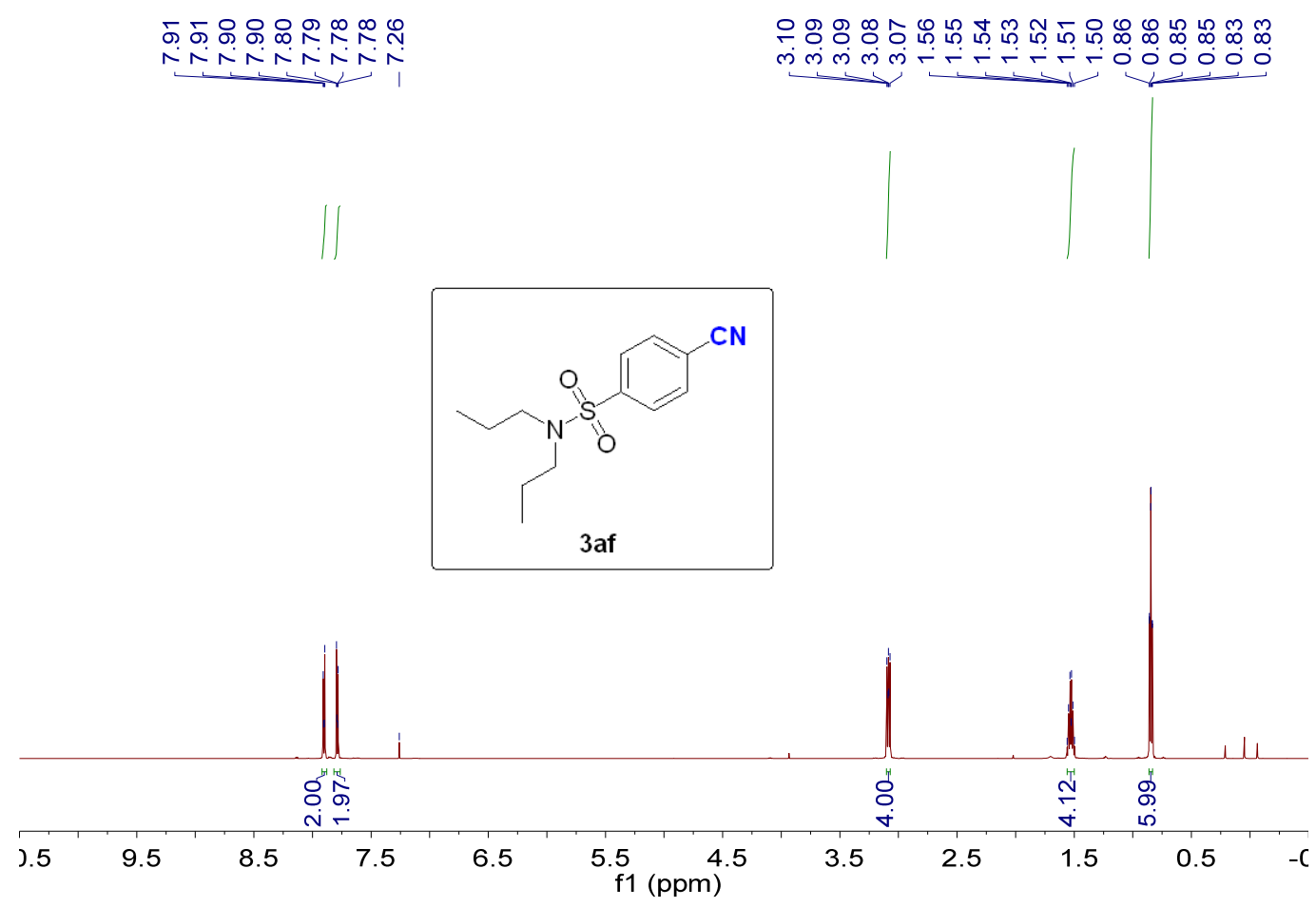

${ }^{1} \mathrm{H}$ NMR $(600 \mathrm{MHz})$ spectrum of $\mathbf{3 a f}\left(\mathrm{CDCl}_{3}, \mathrm{rt}\right)$. 


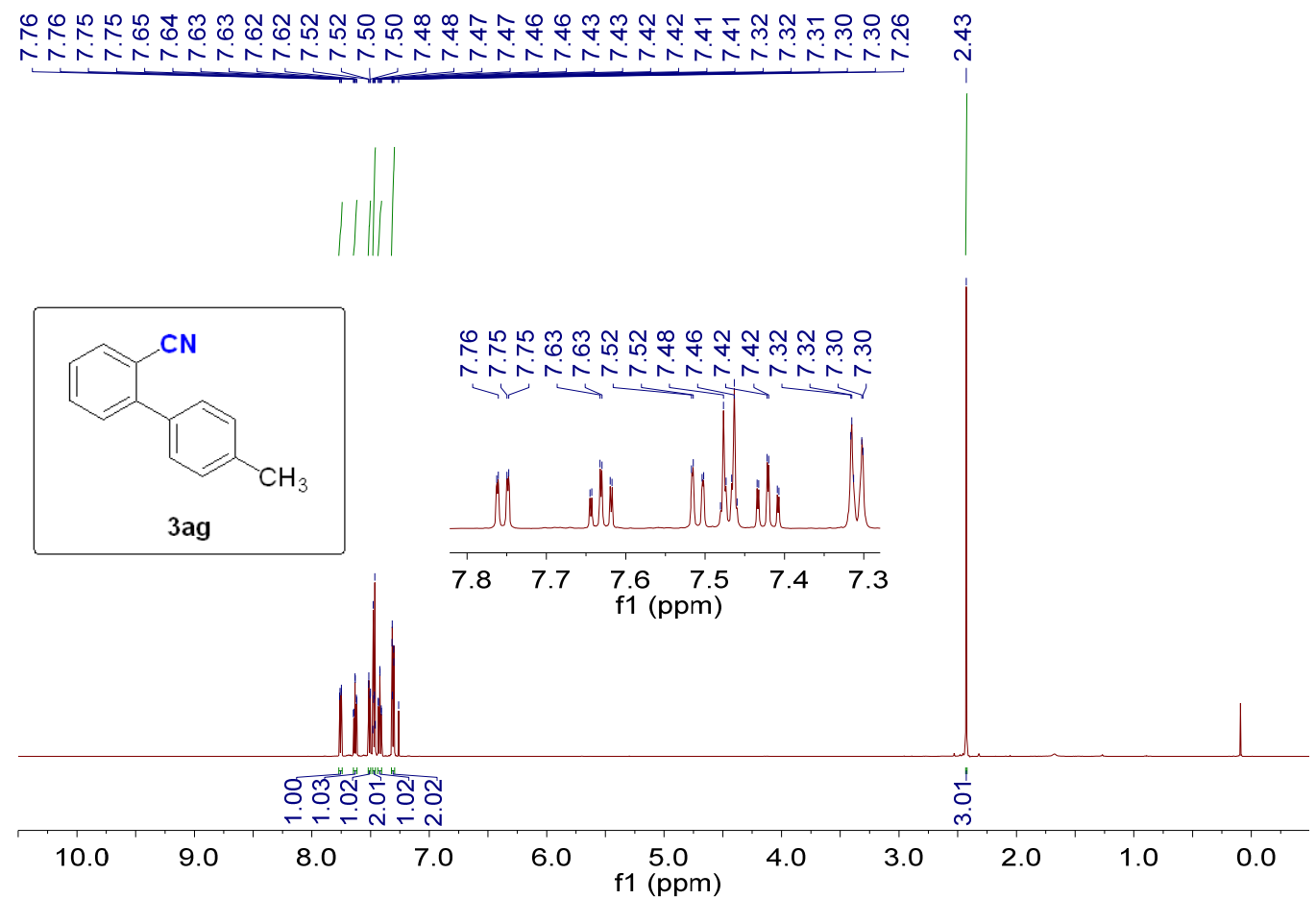

${ }^{1} \mathrm{H}$ NMR $(600 \mathrm{MHz})$ spectrum of $\mathbf{3 a g}\left(\mathrm{CDCl}_{3}, \mathrm{rt}\right)$. 


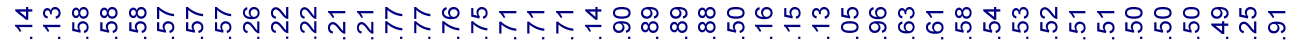

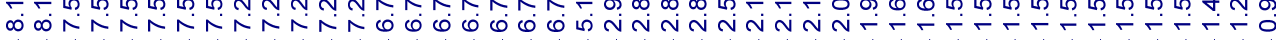

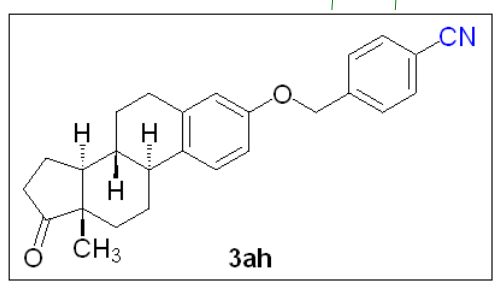

3ah

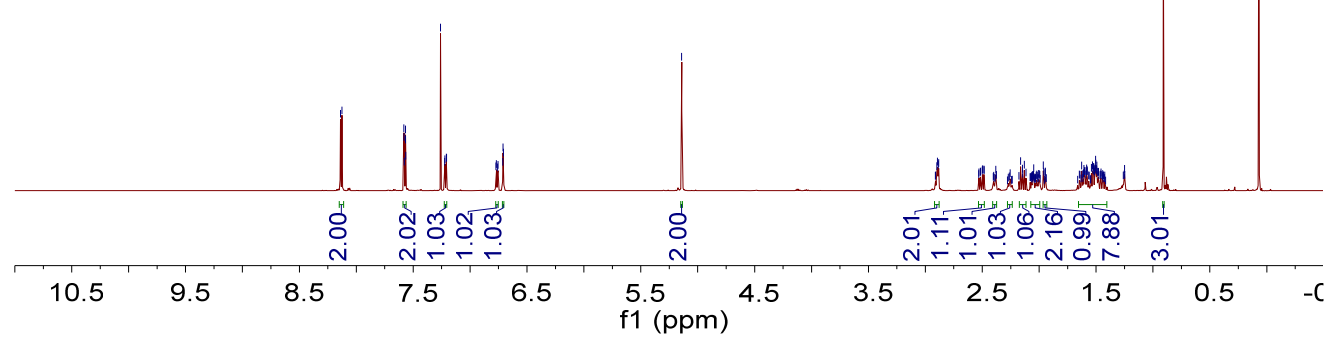

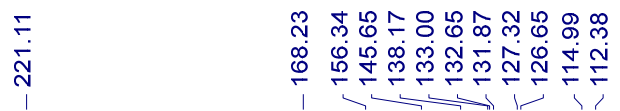

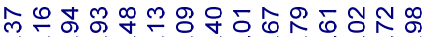

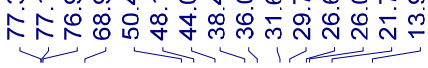

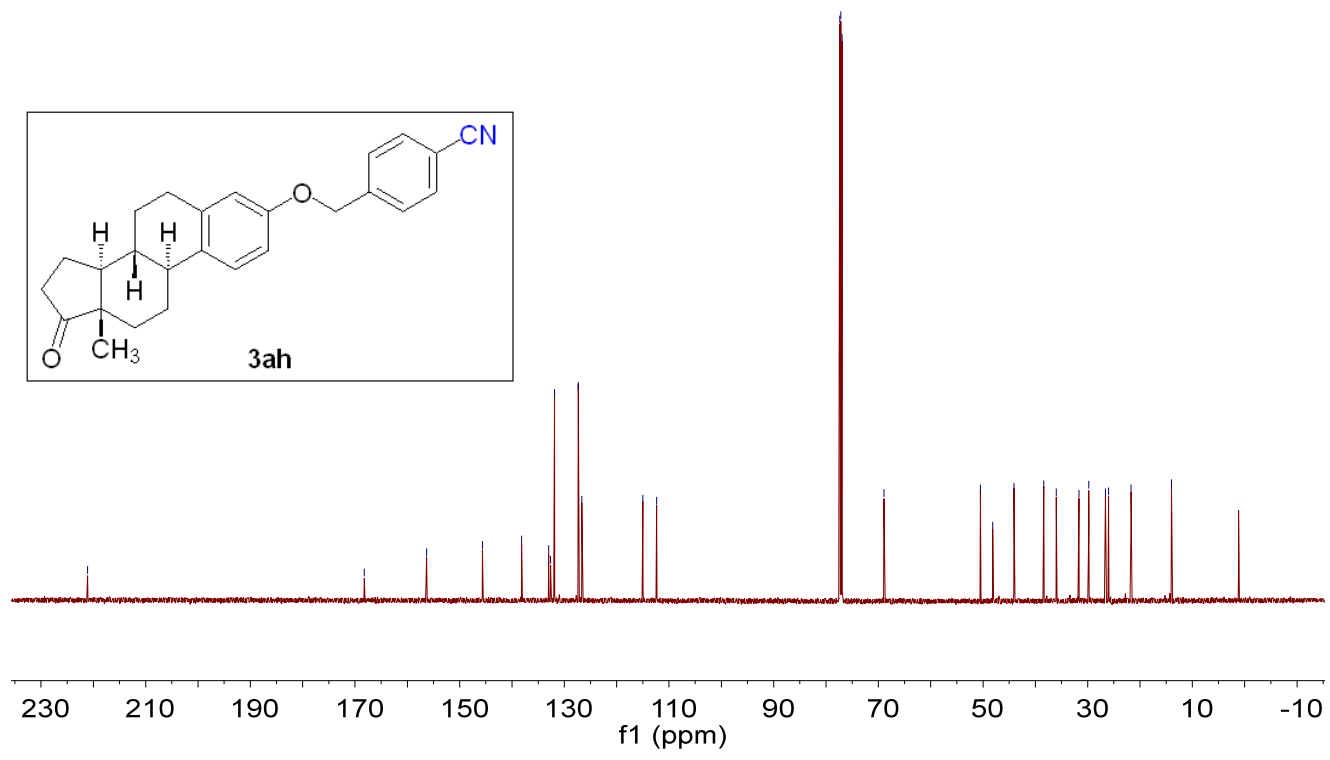

${ }^{1} \mathrm{H}$ NMR $(600 \mathrm{MHz})$ and ${ }^{13} \mathrm{C}\left\{{ }^{1} \mathrm{H}\right\}$ NMR $(151 \mathrm{MHz})$ spectra of 3ah $\left(\mathrm{CDCl}_{3}, \mathrm{rt}\right)$. 


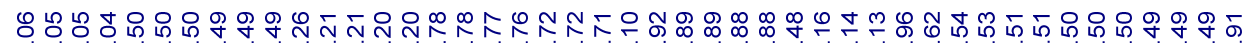
o
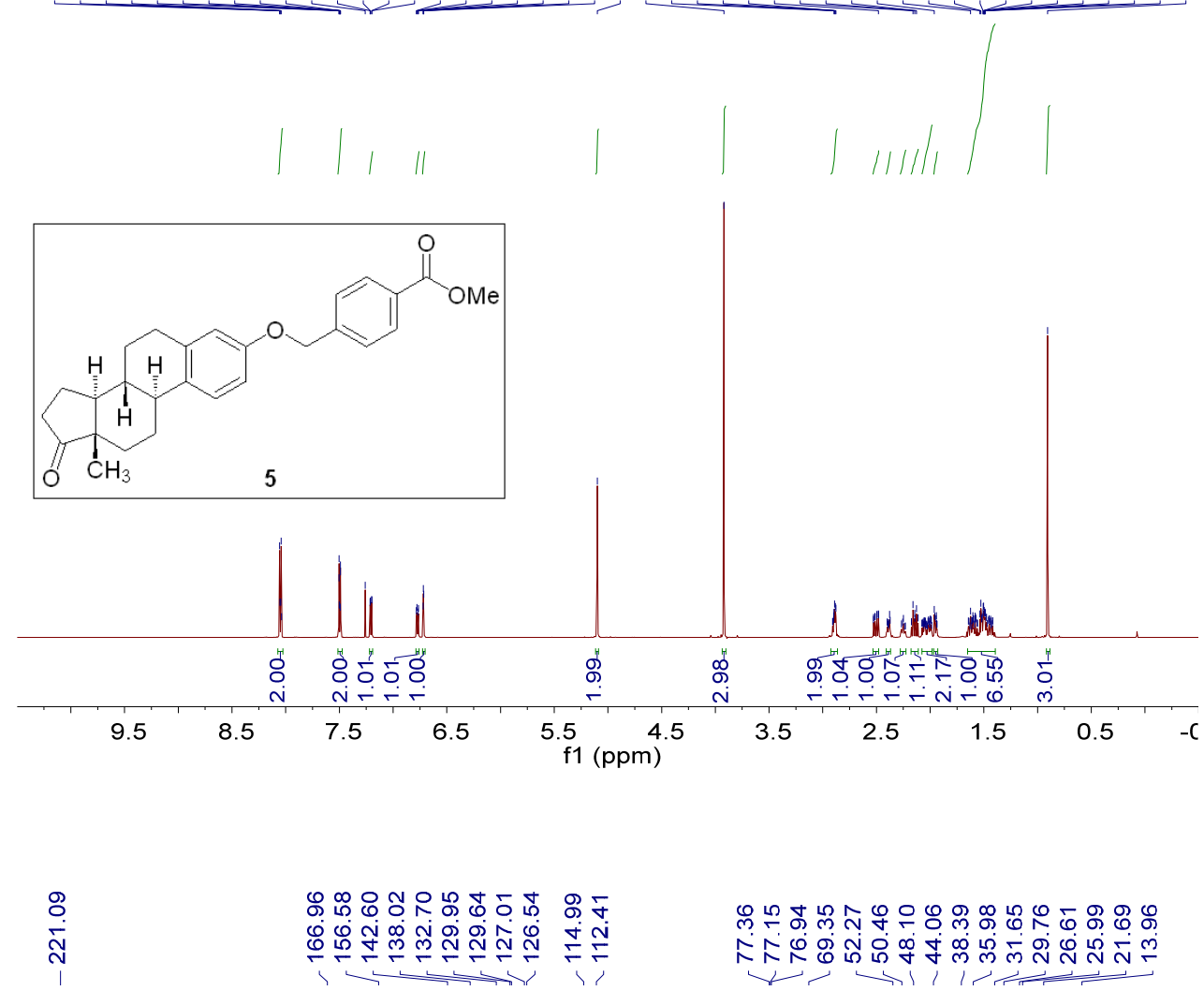

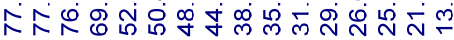
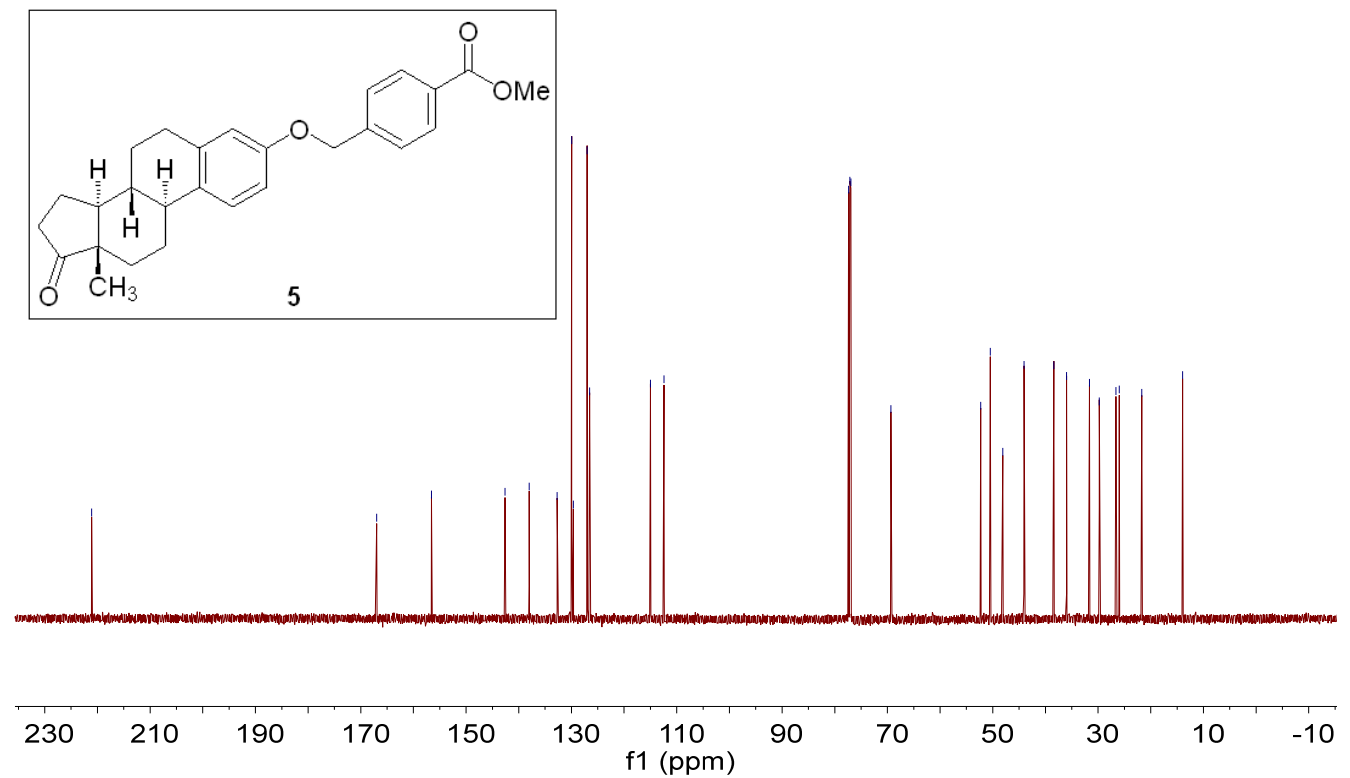

${ }^{1} \mathrm{H}$ NMR $(600 \mathrm{MHz})$ and ${ }^{13} \mathrm{C}\left\{{ }^{1} \mathrm{H}\right\}$ NMR (151 MHz) spectra of $5\left(\mathrm{CDCl}_{3}, \mathrm{rt}\right)$. 


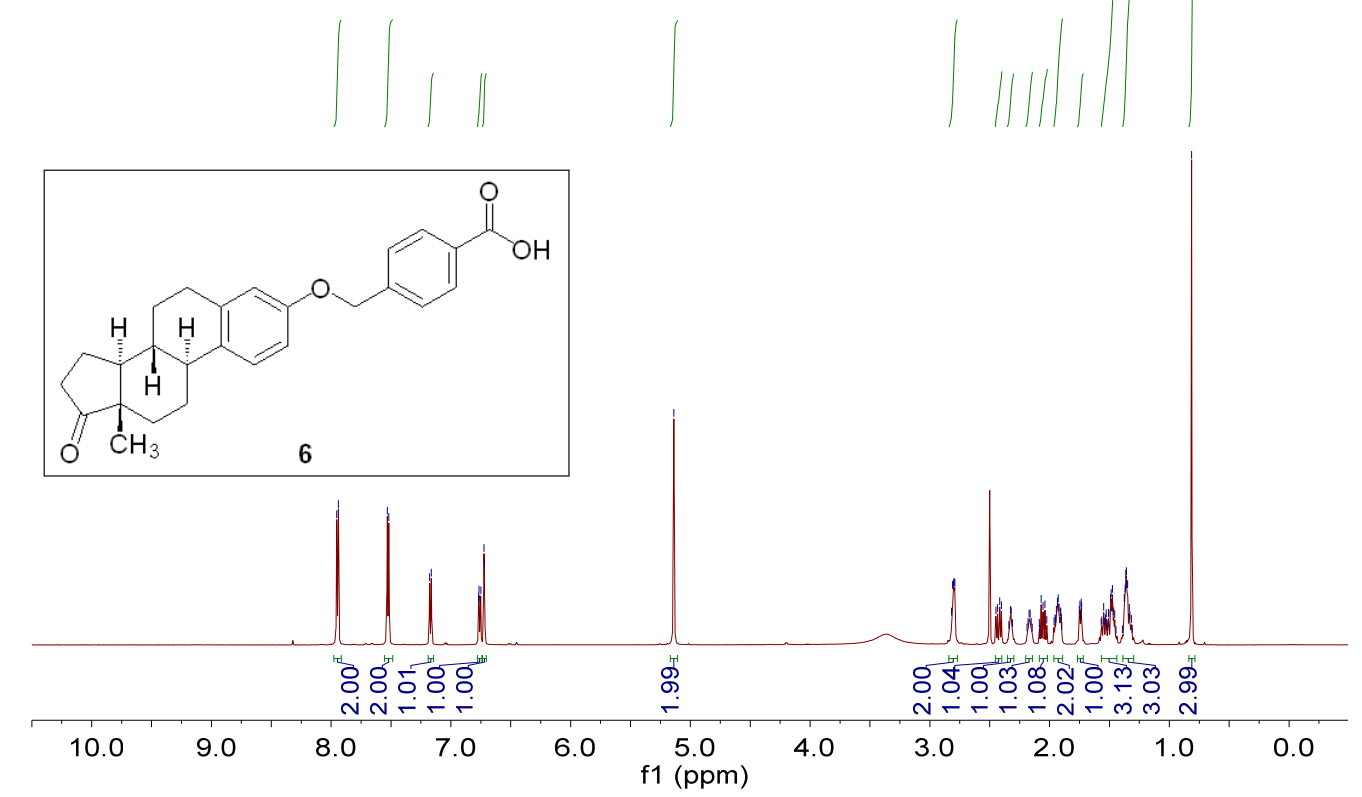

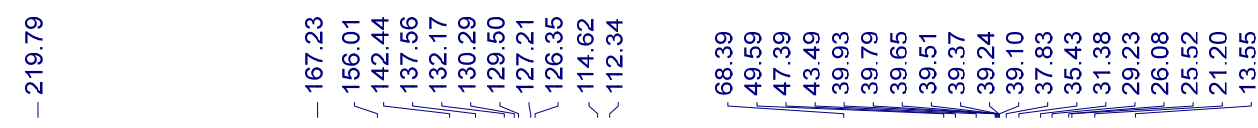
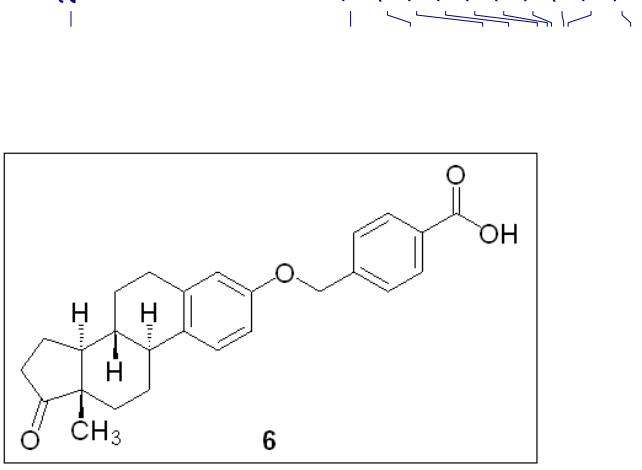

(1) 
サ

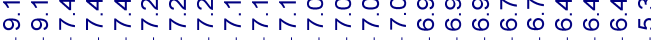
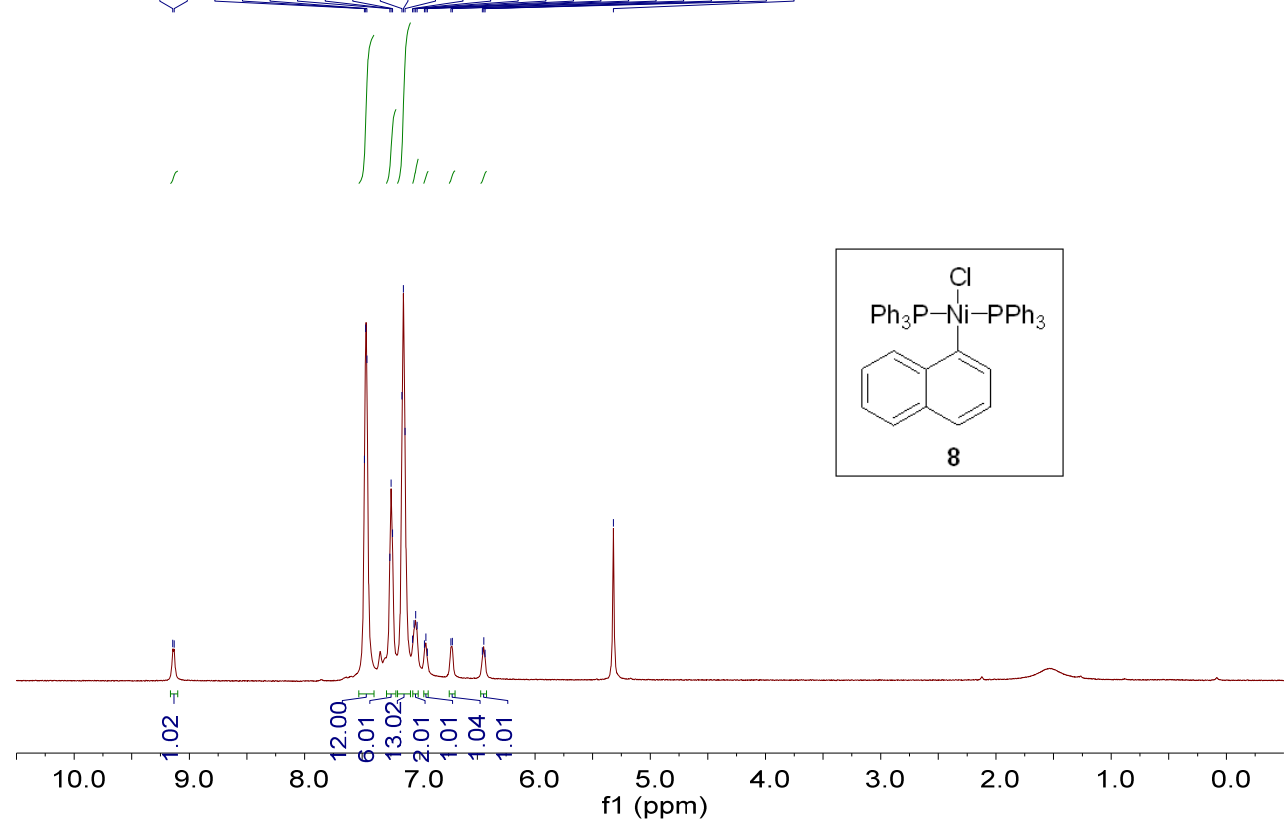

${ }^{1} \mathrm{H}$ NMR $(600 \mathrm{MHz})$ spectrum of $8\left(\mathrm{CD}_{2} \mathrm{Cl}_{2}, \mathrm{rt}\right)$.

$\overline{\grave{n}}$

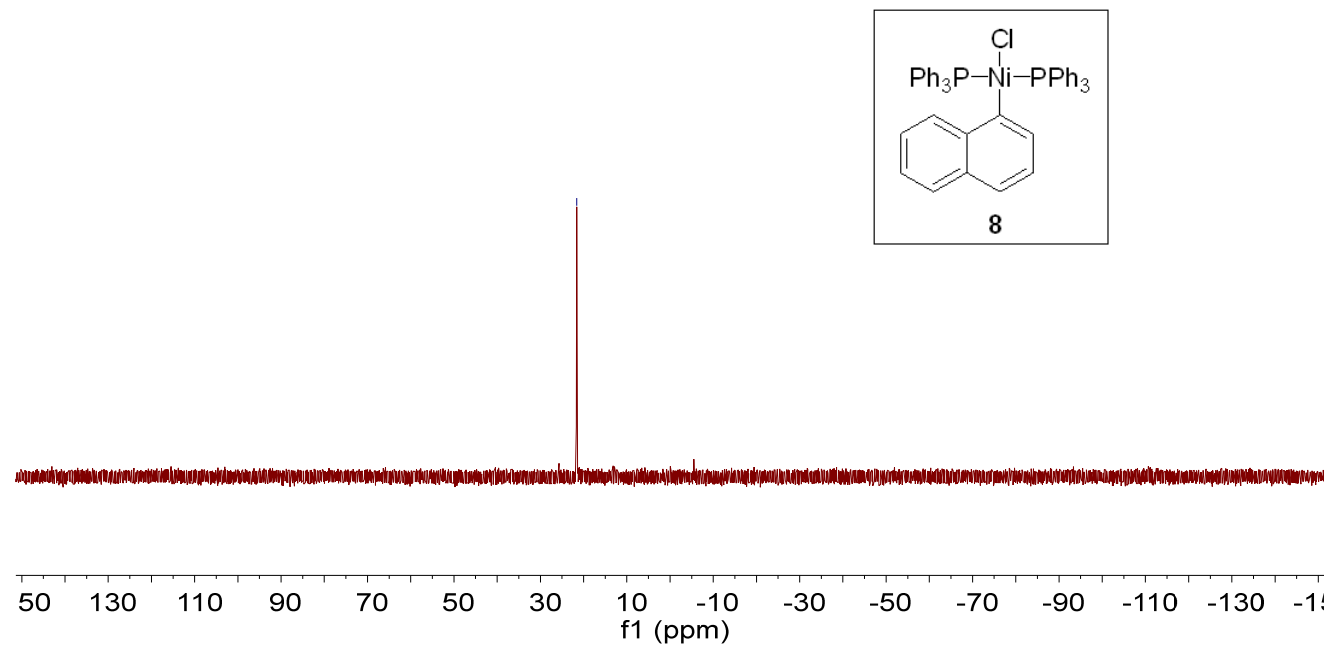

${ }^{31} \mathrm{P}\left\{{ }^{1} \mathrm{H}\right\}$ NMR $(243 \mathrm{MHz})$ spectrum of complex $8\left(\mathrm{C}_{6} \mathrm{D}_{6}, \mathrm{rt}\right)$. 

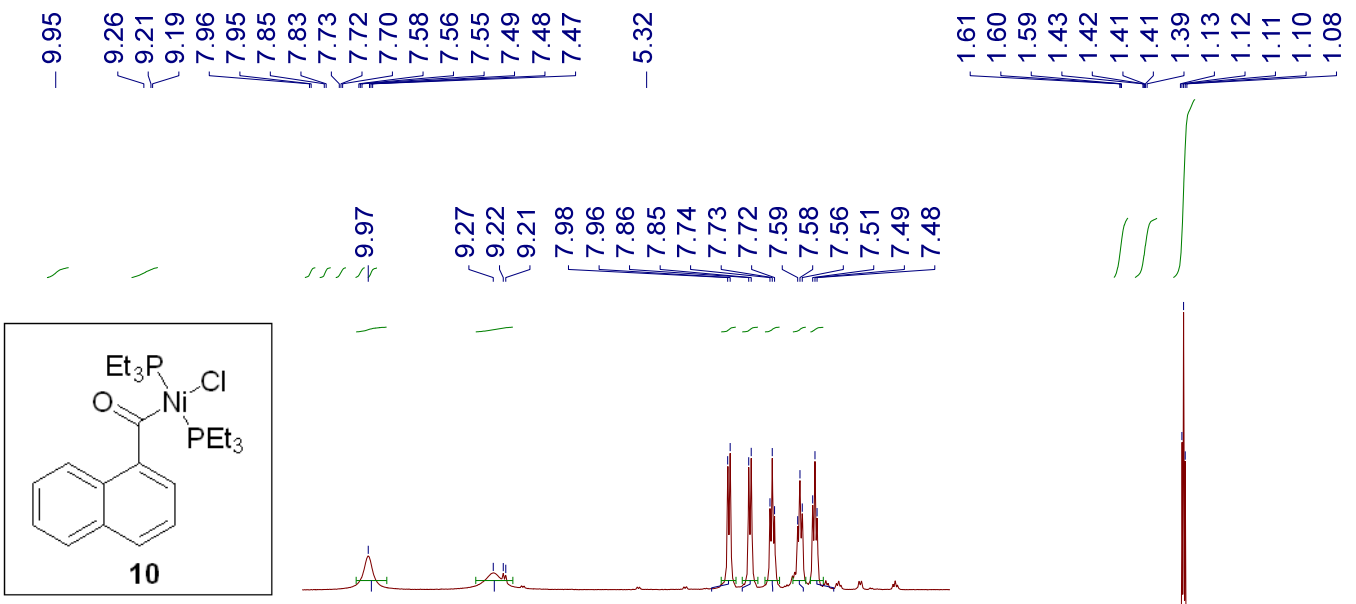

ลำ の

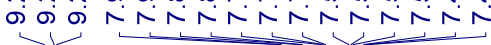

is
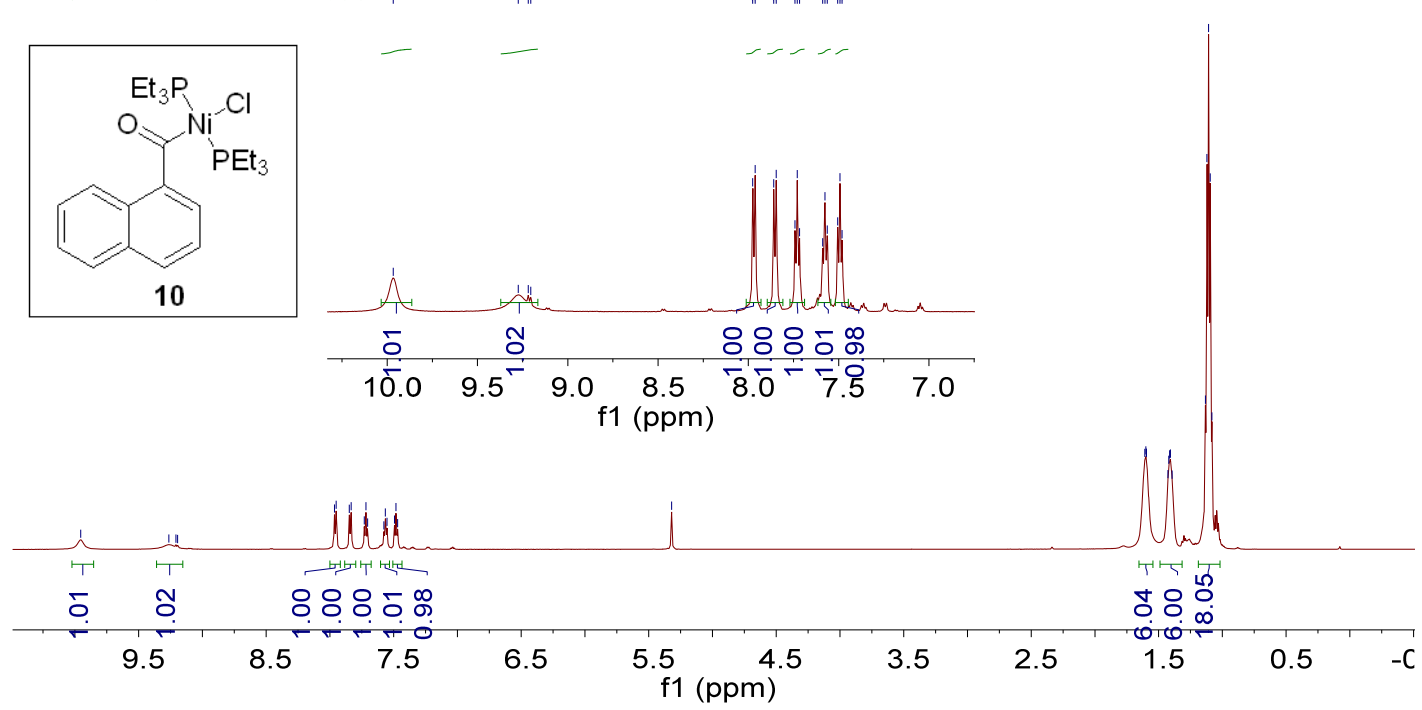

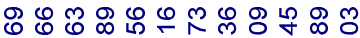

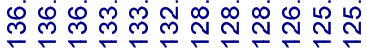

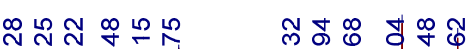

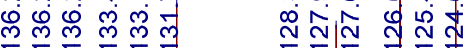
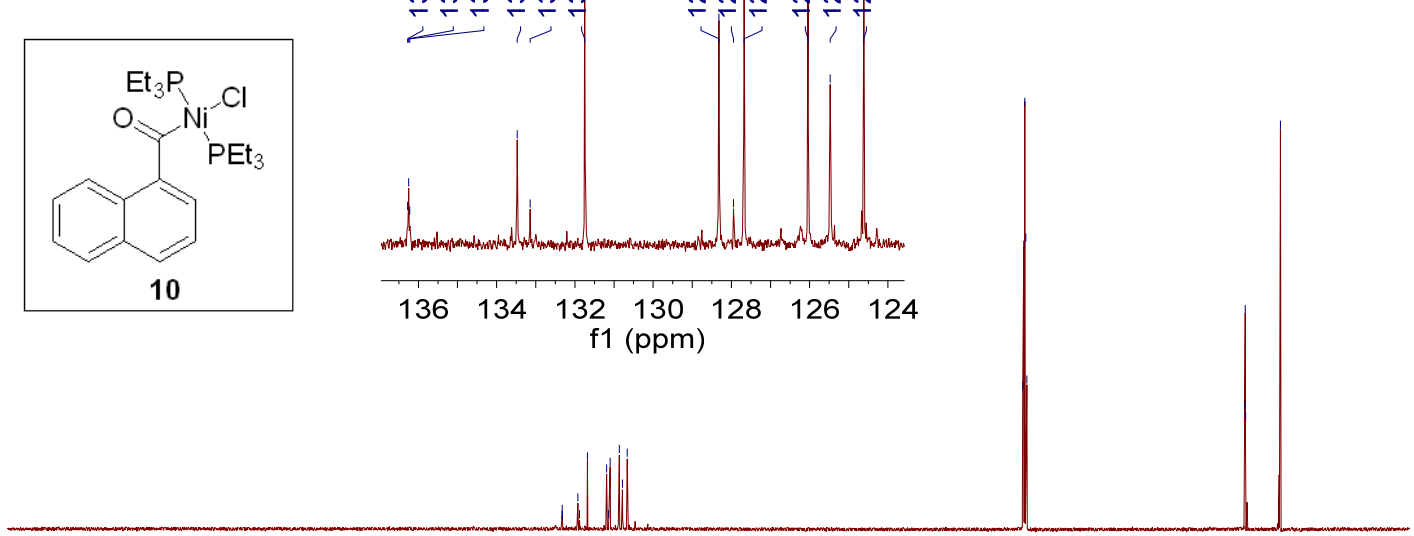

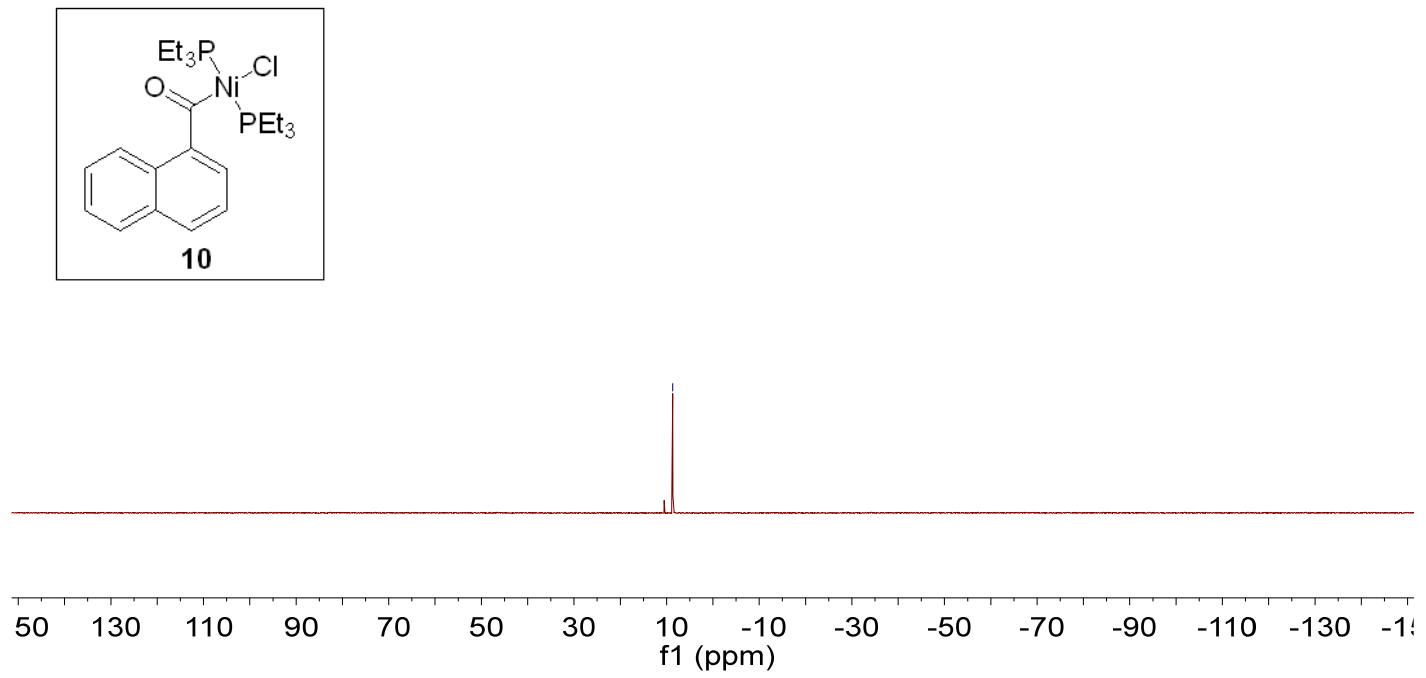

${ }^{1} \mathrm{H}$ NMR (600 MHz), ${ }^{13} \mathrm{C}\left\{{ }^{1} \mathrm{H}\right\}$ NMR $(151 \mathrm{MHz})$ and ${ }^{31} \mathrm{P}\left\{{ }^{1} \mathrm{H}\right\}$ NMR $(243 \mathrm{MHz})$ spectra of complex 10 $\left(\mathrm{CD}_{2} \mathrm{Cl}_{2}, \mathrm{rt}\right)$. 

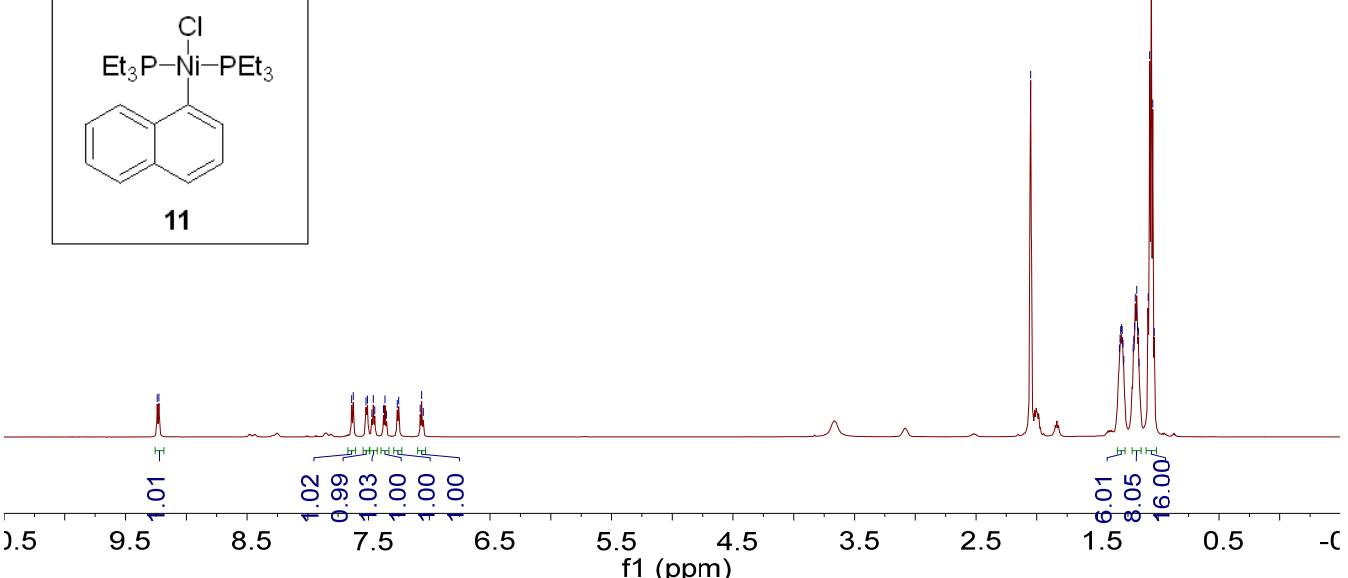

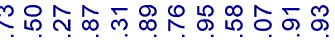

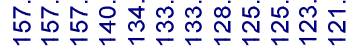

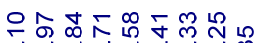

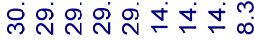

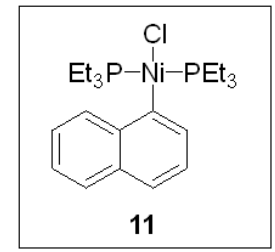

W

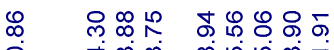

ำ ำ ำ

它它它

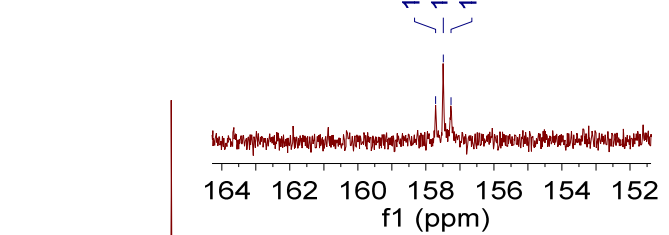

$\mathrm{f1}(\mathrm{ppm})$
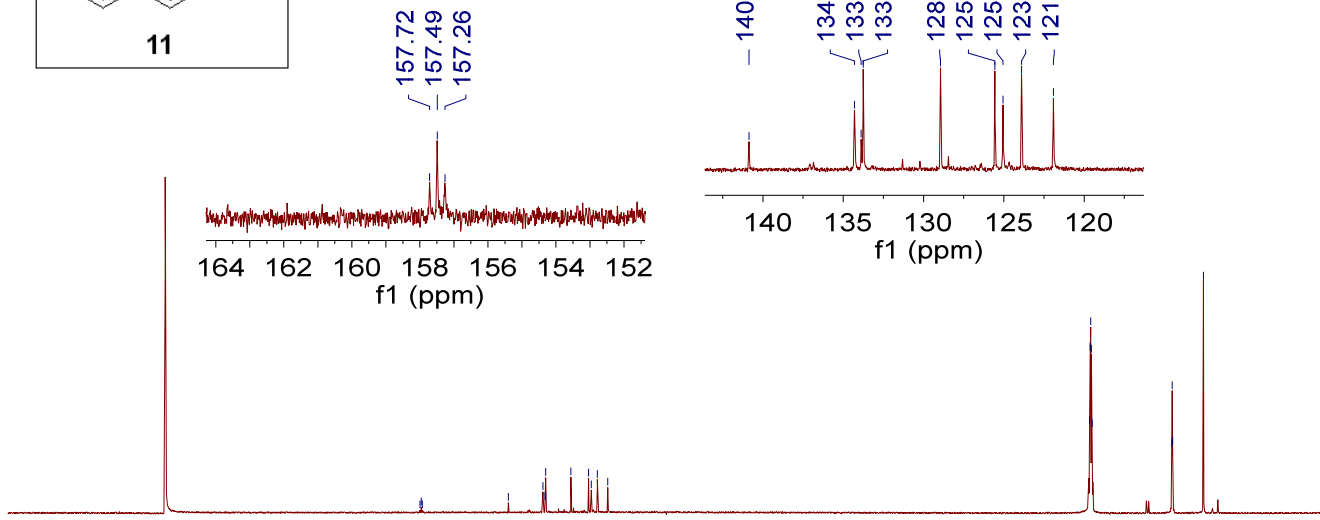

230

210

90170

$50 \quad 130$ f1 (ppm)

${ }^{1} \mathrm{H}$ NMR $(600 \mathrm{MHz})$ and ${ }^{13} \mathrm{C}\left\{{ }^{1} \mathrm{H}\right\} \mathrm{NMR}(151 \mathrm{MHz})$ spectra of $11\left(\left(\mathrm{CD}_{3}\right)_{2} \mathrm{CO}, \mathrm{rt}\right)$. 


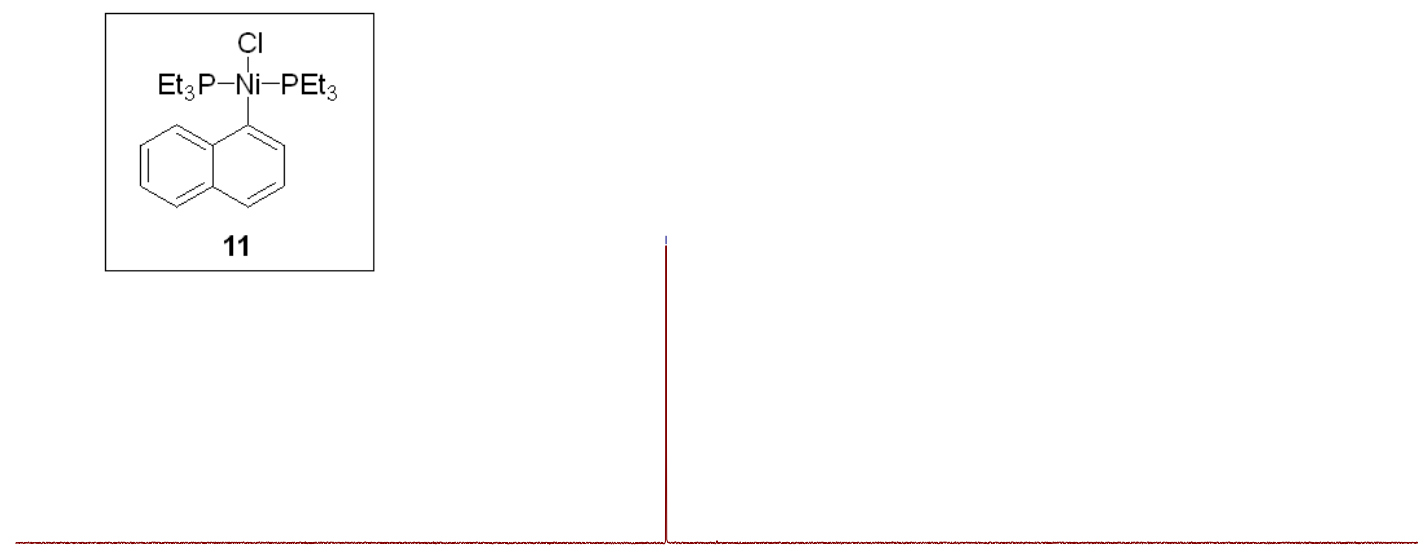

$\begin{array}{lllllllllllllll}50 & 130 & 110 & 90 & 70 & 50 & 30 & \begin{array}{c}10 \\ \mathrm{f} 1(\mathrm{ppm})\end{array} & -30 & -50 & -70 & -90 & -110 & -130 & -1 !\end{array}$

${ }^{31} \mathrm{P}\left\{{ }^{1} \mathrm{H}\right\}$ NMR $(243 \mathrm{MHz})$ spectrum of complex $11\left(\mathrm{C}_{6} \mathrm{D}_{6}, \mathrm{rt}\right)$. 

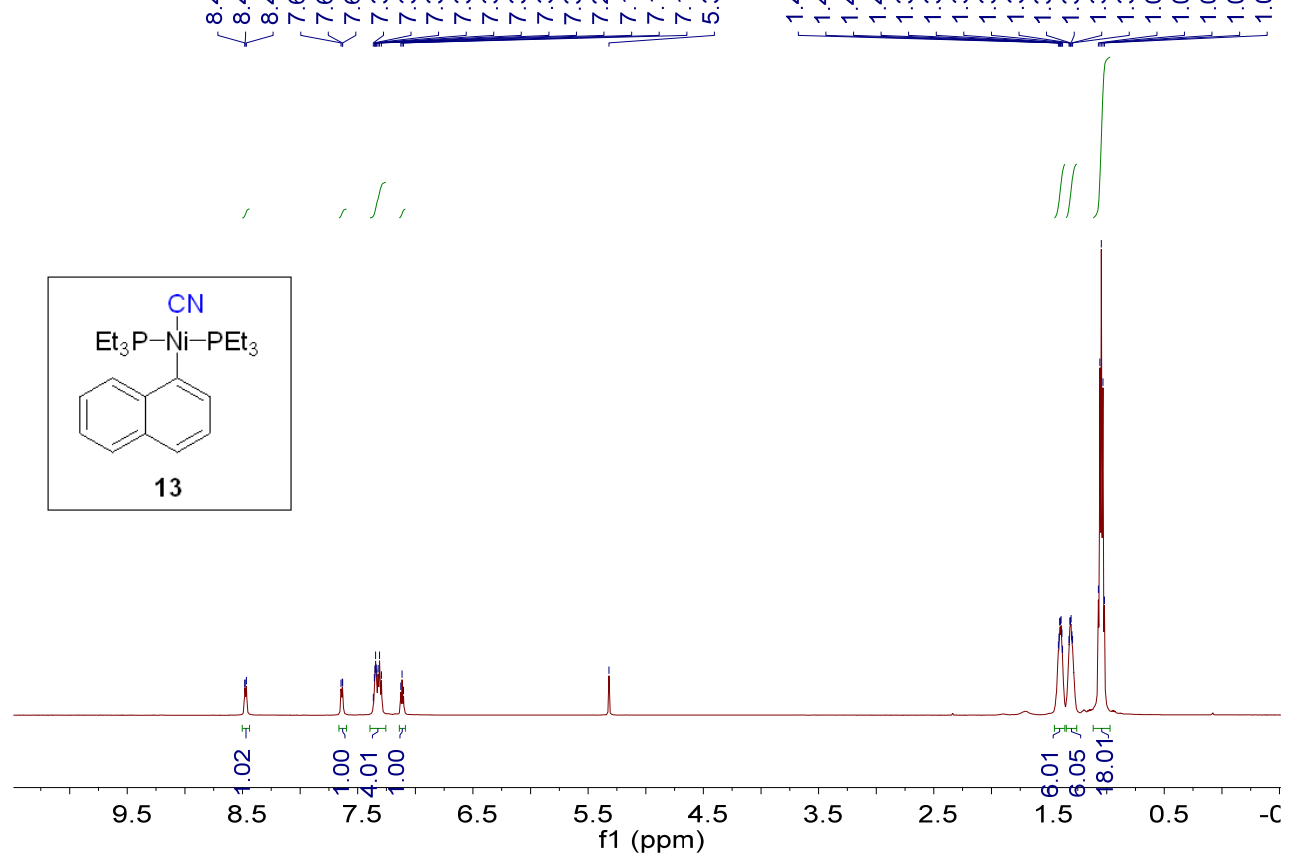

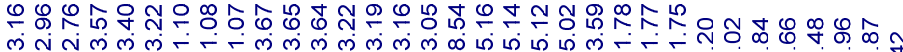

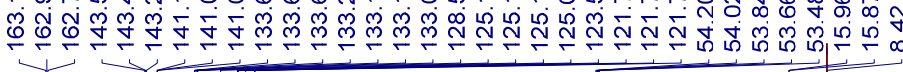

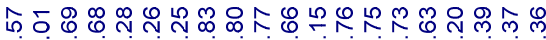

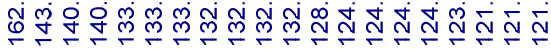

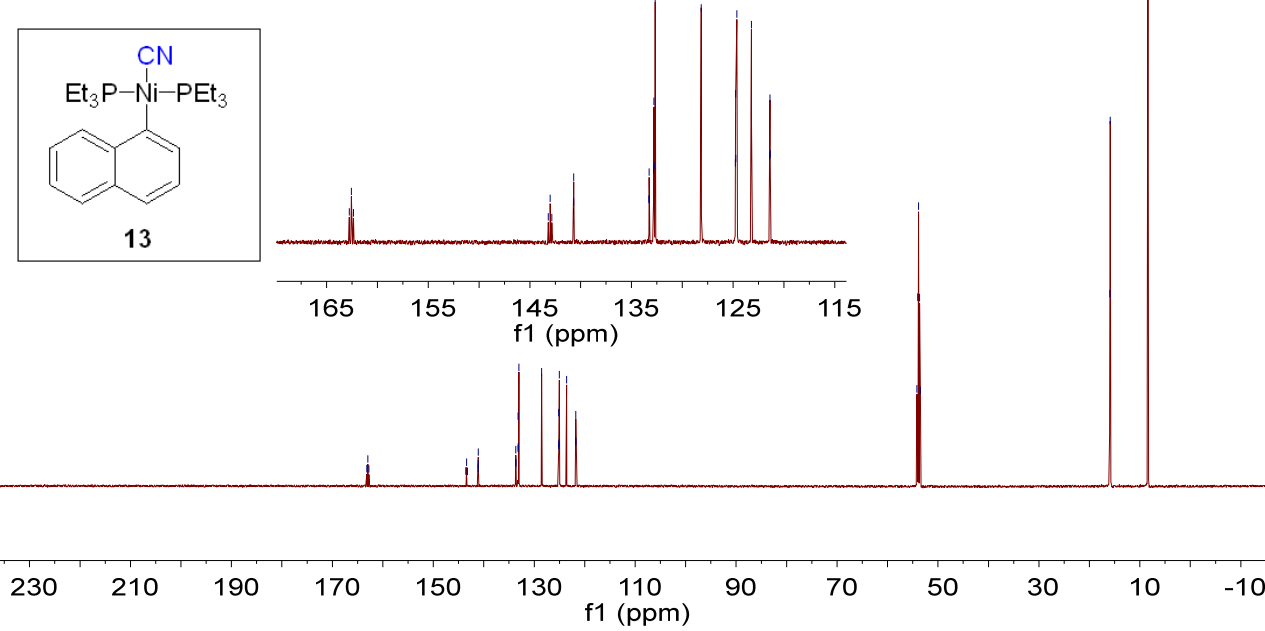




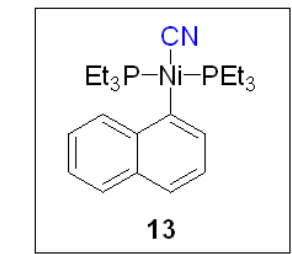

$\begin{array}{lllllllllllllll}50 & 130 & 110 & 90 & 70 & 50 & 30 & \begin{array}{l}10 \\ \mathrm{f} 1(\mathrm{ppm})\end{array} & -30 & -50 & -70 & -90 & -110 & -130 & -1 !\end{array}$

${ }^{1} \mathrm{H}$ NMR (600 MHz), ${ }^{13} \mathrm{C}\left\{{ }^{1} \mathrm{H}\right\}$ NMR (151 MHz), and ${ }^{31} \mathrm{P}\left\{{ }^{1} \mathrm{H}\right\}$ NMR (243 MHz) spectra of complex 13 $\left(\mathrm{CD}_{2} \mathrm{Cl}_{2}, \mathrm{rt}\right)$. 


\section{References}

(1) Shi, S.; Szostak, M. Org. Lett. 2017, 19, 3095-3098.

(2) Gan, Y.; Wang, G.; Xie, X.; Liu, Y. J. Org. Chem. 2018, 83, 14036-14048.

(3) Chatupheeraphat, A.; Liao, H.-H.; Lee, S.-C.; Rueping, M. Org. Lett. 2017, 19, 4255-4258.

(4) Wang, X.; Li, C.; Wang, X.; Wang, Q.; Dong, X.-Q.; Duan, A.; Zhao, W. Org. Lett. 2018, 20, 4267-4272.

(5) Zhu, C.; Chen, F.; Liu, C.; Zeng, H.; Yang, Z.; Wu, W.; Jiang, H. J. Org. Chem. 2018, 83, $14713-$ 14722 .

(6) Zhao, S.; Guo, Y.; Han, E.-J.; Luo, J.; Liu, H.-M.; Liu, C.; Xie, W.; Zhang, W.; Wang, M. Org. Chem. Front. 2018, 5, 1143-1147.

(7) Ding, R.; Liu, Y.; Han, M.; Jiao, W.; Li, J.; Tian, H.; Sun, B. J. Org. Chem. 2018, 83, 1293912944.

(8) Fang, C.; Li, M.; Hu, X.; Mo, W.; Hu, B.; Sun, N.; Jin, L.; Shen, Z. RSC Adv. 2017, 7, 1484-1489.

(9) Malapit, C. A.; Ichiishi, N.; Sanford, M. S. Org. Lett. 2017, 19, 4142-4145.

(10) Mao, Y.; Liu, Y.; Hu, Y.; Wang, L.; Zhang, S.; Wang, W. ACS Catal. 2018, 8, 3016-3020.

(11) Wang, M.; Qiao, Z.; Zhao, J.; Jiang, X. Org. Lett. 2018, 20, 6193-6197.

(12) Kvaskoff, D.; Bednarek, P.; George, L.; Pankajakshan, S.; Wentrup, Curt. J. Org. Chem. 2005, 70, 7947-7955.

(13) Zhou, W.; Xu, L.; Zhang, L.; Jiao, N. Org. Lett.,2010, 12, 2888-2891.

(14) Itaya, M.; Takai, Y.; Kaiya, T. Yakugaku Zasshi 1966, 86, 600-608.

(15) Jiang, B.; Wu, S.; Zeng, J.; Yang, X. Org. Lett. 2018, 20, 6573-6577.

(16) Manos-Turvey, A.; Watson, E. E.; Sykes, M. L.; Jones, A. J.; Baell, J. B.; Kaiser, M.; Avery, V. M.; Payne, R. J. Med. Chem. Commun. 2015, 6, 403-406.

(17) Liu, C.; Ji, C.-L.; Hong, X.; Szostak, M. Angew. Chem., Int. Ed. 2018, 57, 16721-16726.

(18) Kawabata, K.; Takeguchi, M.; Goto, H. Macromolecules 2013, 46, 2078-2091.

(19) Jover, J.; Miloserdov, F. M.; Benet-Buchholz, J.; Grushin, V. V.; Maseras, F. Organometallics 2014, 33, 6531-6543. 